\title{
SYSTEMS OF LOGIC BASED ON ORDINALS $\dagger$
}

\author{
By A. M. TURING.
}

[Received 31 May, 1938.-Kead 16 June, 1938.]

Introduction

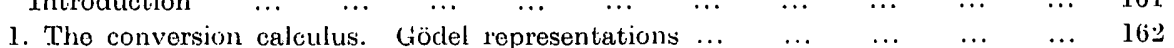

2. Wffective calculability. Abbreviation of treatinent $\quad \ldots \quad \ldots \quad \ldots \quad \ldots \quad$. $\quad \ldots \quad 166$

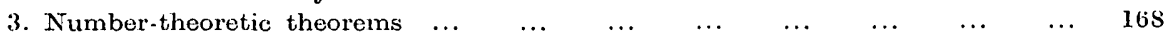

4. A type of problem which is not number-theoretic $\quad \ldots \quad \ldots \quad \ldots \quad \ldots \quad$. $\quad \ldots \quad 172$

5. Syntactical theorems as number-theoretic theorems $\quad \ldots \quad$.

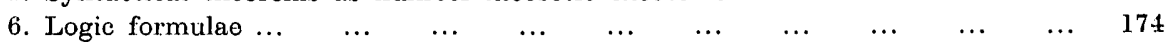

$\begin{array}{lllllllllllll}\text { 7. Ordinals } \ldots & \ldots & \ldots & \ldots & \ldots & \ldots & \ldots & \ldots & \ldots & \ldots & \ldots & 178\end{array}$

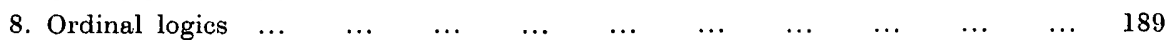

9. Completeness questions $\quad \ldots \quad \begin{array}{lllllllllll} & \ldots & \ldots & \ldots & \ldots & \ldots & \ldots & \ldots & 198\end{array}$

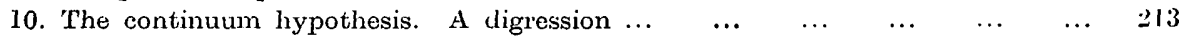

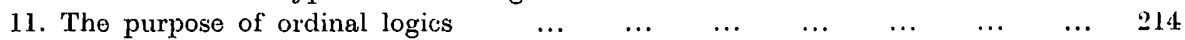

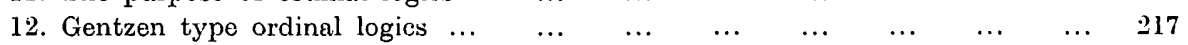

$\begin{array}{llllllllllll}\text { Index of definitions } & \ldots & \ldots & \ldots & \ldots & \ldots & \ldots & \ldots & \ldots & \ldots & 225\end{array}$

$\begin{array}{lllllllllllll}\text { Bibliography } & \ldots & \ldots & \ldots & \ldots & \ldots & \ldots & \ldots & \ldots & \ldots & \ldots & \underline{2} \boldsymbol{s}\end{array}$

The well-known theorem of Gödel (Gödel [1], [2]) shows that every system of logic is in a certain sense incomplete, but at the same time it indicates means whereby from a system $L$ of logic a more complete system $L^{\prime}$ may be obtained. By repeating the process we get a sequence $L, L_{1}=L^{\prime}, L_{2}=L_{1}{ }^{\prime}, \ldots$ each more complete than the preceding. A logic $L_{\omega}$ may then be constructed in which the provable theorems are the totality of theorems provable with the help of the logics $L, L_{1}, L_{2}, \ldots$. We may then form $L_{2 \omega}$ related to $L_{\omega}$ in the same way as $L_{\omega}$ was related to $L$. Proceeding in this way we can associate a system of logic with any constructive ordinalł. It may be asked whether a sequence of logics of this kind is complete in the sense that to any problem $A$ there corresponds

† This paper represents work done while a Jane Eliza Procter Visiting Fellow at Princeton University, where the author received most valuable advice and assistance from Prof. Alonzo Church.

$\ddagger$ The situation is not quite so simple as is suggested by this crude argument. See pages 189-193, 202, 203.

SER. 2. vol. 45 . No. 2239 . 
an ordinal a such that $A$ is solvable by means of the logic $L_{\alpha}$. I propose to investigate this question in a rather more general case, and to give some other examples of ways in which systems of logic may be associated with constructive ordinals.

\section{The calculus of conversion. Gödel representations.}

It will be convenient to be able to use the "conversion calculus" of Church for the description of functions and for some other purposes. This will make greater clarity and simplicity of expression possible. I give a short account of this calculus. For detailed descriptions see Church [3], [2], Kleene [1], Church and Rosser [1].

The formulae of the calculus are formed from the symbols $\{\},,($,$) ,$ 1. $], \lambda, \delta$, and an infinite list of others called variables; we shall take for our infinite list $a, b, \ldots, z, x^{\prime}, x^{\prime \prime}, \ldots$. Certain finite sequences of such symbols are called well-formed formulae (abbreviated to W.F.F.); we define this class inductively, and define simultaneously the free and the bound variables of a W.F.F. Any variable is a W.F.F.; it is its only free variable, and it has no bound variables. $\delta$ is a W.F.F. and has no free or bound variables. If $\mathbf{M}$ and $\mathbf{N}$ are W.F.F. then $\{\mathbf{M}\}(\mathbf{N})$ is a W.F.F., whose free variables are the free variables of $\mathbf{M}$ together with the free variables of $\mathbf{N}$, and whose bound variables are the bound variables of $\mathbf{M}$ together with those of $\mathbf{N}$. If $\mathbf{M}$ is a W.F.F. and $\mathbf{V}$ is one of its free variables, then $\lambda \mathbf{V}[\mathbf{M}]$ is a W.F.F. whose free variables are those of $\mathbf{M}$ with the exception of $\mathbf{V}$, and whose bound variables are those of $\mathbf{M}$ together with $\mathbf{V}$. No sequence of symbols is a W.F.F. except in consequence of these three statements.

In metamathematical statements we use heavy type letters to stand for variable or undetermined formulae, as was done in the last paragraph, and in future such letters will stand for well-formed formulae unless otherwise stated. Small letters in heavy type will stand for formulae representing undetermined positive integers (see below).

A. W.F.F. is said to be in normal form if it has no parts of the form $\{\lambda \mathbf{V}[\mathbf{M}]\}(\mathbf{N})$ and none of the form $\{\{\delta\}(\mathbf{M})\}(\mathbf{N})$, where $\mathbf{M}$ and $\mathbf{N}$ have no free variables.

We say that one W.F.F. is immediately convertible into another if it is obtained from it either by:

(i) Replacing one occurrence of a well-formed part $\lambda \mathbf{V}[\mathbf{M}]$ by $\lambda \mathbf{U}[\mathbf{N}]$, where the variable $\mathbf{U}$ does not occur in $\mathbf{M}$, and $\mathbf{N}$ is obtained from $\mathbf{M}$ by replacing the variable $\mathbf{V}$ by $\mathbf{U}$ throughout. 
(ii) Replacing a well-formed part $\{\lambda \mathbf{V}[\mathbf{M}]\}(\mathrm{N})$ by the formula which is obtained from $\mathbf{M}$ by replacing $\mathbf{V}$ by $\mathbf{N}$ throughout, provided that the bound variables of $\mathbf{M}$ are distinct both from $\mathbf{V}$ and from the free variables of $\mathbf{N}$.

(iii) The process inverse to (ii).

(iv) Replacing a well-formed part $\{\{\delta\}(\mathbf{M})\}(\mathbf{M})$ by

$$
\lambda f[\lambda x[\{f\}(\{f\}(x))]]
$$

if $\mathbf{M}$ is in normal form and has no free variables.

(v) Replacing a well-formed part $\left\{\{\delta\}(\mathbf{M})_{j}^{\prime}(\mathbf{N})\right.$ by

$$
\lambda f[\lambda x[\{f\}(x)]]
$$

if $\mathbf{M}$ and $\mathbf{N}$ are in normal form, are not transformable into one another by repeated application of (i), and have no free variables.

(vi) The process inverse to (iv).

(vii) The process inverse to (v).

These rules could have been expressed in such a way that in no case could there be any doubt about the admissibility or the result of the transformation [in particular this can be done in the case of process (v)].

A formula $\mathbf{A}$ is said to be convertible into another $\mathbf{B}$ (abbreviated to "A conv B") if there is a finite chain of immediate conversions leading from one formula to the other. It is easily seen that the relation of convertibility is an equivalence relation, i.e. it is symmetric, transitive, and reflexive.

Since the formulae are liable to be very lengthy, we need means for abbreviating them. If we wish to introduce a particular letter as an abbreviation for a particular lengthy formula we write the letter followed by " $\rightarrow$ " and then by the formula, thus

$$
I \rightarrow \lambda x[x]
$$

indicates that $I$ is an abbreviation for $\lambda x[x]$. We also use the arrow in less sharply defined senses, but never so as to cause any real confusion. In these cases the meaning of the arrow may be rendered by the words "stands for". 
If a formula $F$ is, or is represented by, a single symbol we abbreviate $\{\mathbf{F}\}(\mathbf{X})$ to $\mathbf{F}(\mathbf{X})$. A formula $\{\{\mathbf{F}\}(\mathbf{X})\}(\mathbf{Y})$ may be abbreviated to

$$
\{\mathbf{F}\}(\mathbf{X}, \mathbf{Y}) \text {, }
$$

or to $\mathbf{F}(\mathbf{X}, \mathbf{Y})$ if $\mathbf{F}$ is, or is represented by, a single symbol. Similarly for $\left\{\left\{\{\mathbf{F}\}(\mathbf{X})_{j}(\mathbf{Y})_{j}(\mathbf{Z})\right.\right.$, etc. A formula $\lambda \mathbf{V}_{1}\left[\lambda \mathbf{V}_{2} \ldots\left[\lambda \mathbf{V}_{r}[\mathbf{M}]\right] \ldots\right]$ may be abbreviated to $\lambda \mathbf{V}_{1} \mathbf{V}_{2} \ldots \mathbf{V}_{r}$. $\mathbf{M}$.

We have not as yet assigned any meanings to our formulae, and we do not intend to do so in general. An exception may be made for the case of the positive integers, which are very conveniently represented by the for'mulae $\lambda f x \cdot f(x), \lambda f x . f(f(x)), \ldots$. In fact we introduce the abbreviations

$$
\begin{aligned}
& 1 \rightarrow \lambda f: x \cdot f(x) \\
& 2 \rightarrow \lambda f x \cdot f(f(x)) \\
& 3 \rightarrow \lambda f x \cdot f(f(f(x))), \quad \text { etc., }
\end{aligned}
$$

and we also say, for example, that $\lambda f x . f(f(x))$, or in full

$$
\lambda f[\lambda x[\{f\}(\{f\}(x))]],
$$

represents the positive integer 2. Later we shall allow certain formulae to represent ordinals, but otherwise we leave them without explicit meaning; an implicit meaning may be suggested by the abbreviations used. In any case where any meaning is assigned to formulae it is desirable that the meaning should be invariant under conversion. Our definitions of the positive integers do not violate this requirement, since it may be proved that no two formulae representing different positive integers are convertible the one into the other.

In connection with the positive integers we introduce the abbreviation

$$
S \rightarrow \lambda u f x . f(u(f, x)) .
$$

This formula has the property that, if $\mathbf{n}$ represents a positive integer, $S(\mathbf{n})$ is convertible to a formula representing its successor $\dagger$.

Formulae representing undetermined positive integers will be represented by small letters in heavy type, and we adopt once for all the 
convention that, if a small letter, $n$ say, stands for a positive integer, then the same letter in heavy type, $\mathbf{n}$, stands for the formula representing the positive integer. When no confusion arises from so doing, we shall not trouble to distinguish between an integer and the formula which represents it.

Suppose that $f(n)$ is a function of positive integers taking positive integers as values, and that there is a W.F.F. F not containing $\delta$ such that, for each positive integer $n, \mathbf{F}(\mathbf{n})$ is convertible to the formula representing $f(n)$. We shall then say that $f(n)$ is $\lambda$-definable or formally definable, and that $\mathbf{F}$ formally defines $f(n)$. Similar conventions are used for functions of more than one variable. The sum function is, for instance, formally defined by $\lambda a b f x . a(f, b(f, x))$; in fact, for any positive integers $m, n, p$ for which $m+n=p$, we have

$$
\{\lambda a b f x \cdot a(f, b(f, x))\}(\mathbf{m}, \mathbf{n}) \text { conv } \mathbf{p} .
$$

In order to emphasize this relation we introduce the abbreviation

$$
\left.\mathbf{X}+\mathbf{Y} \rightarrow i_{i}^{\prime} \lambda a b f x . a(f, b(f, x))\right\}_{j}(\mathbf{X}, \mathbf{Y})
$$

and we shall use similar notations for sums of three or more terms, products, etc.

For any W.F.F. G we shall say that $\mathbf{G}$ enumerates the sequence $\mathbf{G}(1), \mathbf{G}(2), \ldots$ and any other sequence whose terms are convertible to those of this sequence.

When a formula is convertible to another which is in normal form, the second is described as a normal form of the first, which is then said to have a normal form. I quote here some of the more important theorems concerning normal forms.

(A) If a formula has two normal forms they are convertible into one another by the use of (i) alone. (Church and Rosser [1], 479, 481.)

(B) If a formula has a normal form then every well-formed part of il has a normal form. (Church and Rosser [1], 480-481.)

(C) There is (demonstrably) no process whereby it can be said of a formuln whether it has a normal form. (Church [3], 360, Theorem XVIII.)

We often need to be able to describe formulae by means of positive integers. The method used here is due to Gödel (Gödel [1]). To each single symbol $s$ of the calculus we assign an integer $r[s]$ as in the table below.

\begin{tabular}{|c|c|c|c|c|c|c|c|c|c|c|c|}
\hline$s$ & $\{$, , or [ & \}, ), or $]$ & $\lambda$ & $\delta$ & $a$ & $\ldots$ & $z$ & $x^{\prime}$ & $x^{\prime \prime}$ & $x^{\prime \prime \prime}$ & $\ldots$ \\
\hline$r[s]$ & 1 & 2 & 3 & 4 & 5 & $\ldots$ & 30 & 31 & 32 & 33 & $\ldots$ \\
\hline
\end{tabular}


If $s_{1}, s_{2}, \ldots, s_{k}$ is a sequence of symbols, then $2^{r\left[s_{1}\right]} 3^{r\left[\varepsilon_{2}\right]} \ldots p_{k}^{r\left[\varepsilon_{k}\right]}$ (where $p_{k}$ is the $k$-th prime number) is called the Gödel representation (G.R.) of that sequence of symbols. No two W.F.F. have the same G.R.

Two theorems on G.R. of W.F.F. are quoted here.

(D) There is a W.F.F. "form" such that if $a$ is the G.R. of a W.F.F. A without free variables, then form (a) conv A. (This follows from a similar theorem to be found in Church [3],53 66. Metads are used there in place of G.R.)

(E) There is a W.F.F. Gr such that, if $\mathbf{A}$ is a W.F.F. with a normal form without free variables, then $\operatorname{Gr}(\mathbf{A})$ conv $\mathbf{a}$, where $a$ is the G.R. of a normal form of A. [Church [3], 53, 66, as (D).]

\section{Effective calculability. Abbreviation of treatment.}

A function is said to be "effectively calculable" if its values can be found by some purely mechanical process. Although it is fairly easy to get an intuitive grasp of this idea, it is nevertheless desirable to have some more definite, mathematically expressible definition. Such a definition was first given by Gödel at Princeton in 1934 (Gödel [2], 26), following in part an unpublished suggestion of Herbrand, and has since been developed by Kleene [2]). These functions were described as "general recursive" by Gödel. We shall not be much concerned here with this particular definition. Another definition of effective calculability has been given by Church (Church [3], 356-358), who identifies it with $\lambda$-definability. The author has recently suggested a definition corresponding more closely to the intuitive idea (Turing [1], see also Post [1]). It was stated above that "a function is effectively calculable if its values can be found by some purely mechanical process ". We may take this statement literally, understanding by a purely mechanical process one which could be carried out by a machine. It is possible to give a mathematical description, in a certain normal form, of the structures of these machines. The development of these ideas leads to the author's definition of a computable function, and to an identification of computability $\dagger$ with effective calculability. It is not difficult, though somewhat laborious, to prove that these three definitions are equivalent (Kleene [3], Turing [2]).

$\dagger$ We shall use the expression "computable function" to mean a function calculable by a machine, and we let " effectively calculable" refer to the intuitive idea without particular identification with any one of these definitions. We do not restrict the values taken by a computable function to be natural numbers; we may for instance have computable propositional functions. 
In the present paper we shall make considerable use of Church's identification of effective calculability with $\lambda$-definability, or, what comes to the same thing, of the identification with computability and one of the equivalence theorems. In most cases where we have to deal with an effectively calculable function, we shall introduce the corresponding W.F.F. with some such phrase as "the function $f$ is effectively calculable, let $F$ be a formula $\lambda$ defining it", or "let $F$ be a formula such that $F(\mathbf{n})$ is convertible to ... whenever $\mathbf{n}$ represents a positive integer". In such cases there is no difficulty in seeing how a machine could in principle be designed to calculate the values of the function concerned; and, assuming this done, the equivalence theorem can be applied. A statement of what the formula $F$ actually is may be omitted. We may immediately introduce on this basis a W.F.F. $\sigma$ with the property that

$$
\varpi(\mathbf{m}, \mathbf{n}) \text { conv } \mathbf{r} \text {, }
$$

if $r$ is the greatest positive integer, if any, for which $m^{r}$ divides $n$ and $r$ is 1 if there is none. We also introduce $\mathrm{Dt}$ with the properties

$$
\begin{array}{r}
\operatorname{Dt}(\mathbf{n}, \mathbf{n}) \text { conv } 3, \\
\operatorname{Dt}(\mathbf{n}+\mathbf{m}, \mathbf{n}) \text { conv } 2, \\
\operatorname{Dt}(\mathbf{n}, \mathbf{n}+\mathbf{m}) \text { conv } 1 .
\end{array}
$$

There is another point to be made clear in connection with the point of view that we are adopting. It is intended that all proofs that are given should be regarded no more critically than proofs in classical analysis. The subject matter, roughly speaking, is constructive systems of logic, but since the purpose is directed towards choosing a particular constructive system of logic for practical use, an attempt at this stage to put our theorems into constructive form would be putting the cart before the horse.

Those computable functions which take only the values 0 and 1 are of particular importance, since they determine and are determined by computable properties, as may be seen by replacing " 0 " and " 1 " by "true" and "false". But, besides this type of property, we may have to consider a different type, which is, roughly speaking, less constructive than the computable properties, but more so than the general predicates of classical mathematics. Suppose that we have a computable function of the natural numbers taking natural numbers as values, then corresponding to this function there is the property of being a value of the function. Such a property we shall describe as "axiomatic"; the reason for using this term is that it is possible to define such a property by giving a set of axioms, the property to hold for a given argument if and only if it is possible to deduce that it holds from the axioms. 
Axiomatic properties may also be characterized in this way. A property $\psi$ of positive integers is axiomatic if and only if there is a computable property $\phi$ of two positive integers, such that $\psi(x)$ is true if and only if there is a positive integer $y$ such that $\phi(x, y)$ is true. Or again $\psi$ is axiomatic if and only if there is a W.F.F. F such that $\psi(n)$ is true if and only if $\mathbf{F}(\mathbf{n})$ conv 2 .

\section{Number-theoretic theorems.}

By a number-theoretic theorem $\uparrow$ we shall mean a theorem of the form " $\theta(x)$ vanishes for infinitely many natural numbers $x$ ", where $\theta(x)$ is a primitive recursive function.

We shall say that a problem is number-theoretic if it has been shown that any solution of the problem may be put in the form of a proof of one or more number-theoretic theorems. More accurately we may say that a class of problems is number-theoretic if the solution of any one of them can be transformed (by a uniform process) into the form of proofs of number-theoretic theorems.

I shall now draw a few consequences from the definition of "number theoretic theorems", and in section 5 I shall try to justify confining our consideration to this type of problem.

$\dagger$ I believe that there is no generally accepted meaning for this term, but it should be noticed that we are using it in a rather restricted sense. The most generally accepted meaning is probably this: suppose that we take an arbitrary formula of the functional calculus of the first order and replace the function variables by primitive recursive relations. The resulting formula represents a typical number-theoretic theorem in this (more general) sense.

$\ddagger$ Primitive recursive functions of natural numbers are defined inductively as follows. Suppose that $f\left(x_{1}, \ldots, x_{n-1}\right), g\left(x_{1}, \ldots, x_{n}\right), h\left(x_{1}, \ldots, x_{n+1}\right)$ are primitive recursive, then $\phi\left(x_{1}, \ldots, x_{n}\right)$ is primitive recursive if it is defined by one of the sets of equations $(a)$ to $(e)$.

(a) $\phi\left(x_{1}, \ldots, x_{n}\right)=h\left(x_{1}, \ldots, x_{m-1}, g\left(x_{1}, \ldots, x_{n}\right), x_{m+1}, \ldots, x_{n-1}, x_{m}\right) \quad(1 \leqslant m \leqslant n)$;

(b) $\phi\left(x_{1}, \ldots, x_{n}\right)=f\left(x_{n}, \ldots, x_{n}\right)$; .

(c) $\phi\left(x_{1}\right)=a$, where $n=1$ and $a$ is some particular natural number;

(d) $\phi\left(x_{1}\right)=x_{1}+1 \quad(n=1)$;

(e) $\phi\left(x_{1}, \ldots, x_{n-1}, 0\right)=f\left(x_{1}, \ldots, x_{n-1}\right)$;

$\phi\left(x_{1}, \ldots, x_{n-1}, x_{n}+1\right)=h\left(x_{1}, \ldots, x_{n}, \phi\left(x_{1}, \ldots, x_{n}\right)\right)$.

The class of primitive recursive functions is more restricted than the class of computable functions, but it has the advantage that there is a process whereby it can be said of a set of equations whether it defines a primitive recursive function in the manner described above.

If $\phi\left(x_{1}, \ldots, x_{n}\right)$ is primitive recursive, then $\phi\left(x_{1}, \ldots, x_{n}\right)=0$ is described as a primitive recursive relation between $x_{1}, \ldots, x_{n}$. 
An alternative form for number-theoretic theorems is "for each natural number $x$ there exists a natural number $y$ such that $\phi(x, y)$ vanishes", where $\phi(x, y)$ is primitive recursive. In other words, there is a rule whereby, given the function $\theta(x)$, we can find a function $\phi(x, y)$, or given $\phi(x, y)$, we can find a function $\theta(x)$, such that " $\theta(x)$ vanishes infinitely often" is a necessary and sufficient condition for "for each $x$ there is a $y$ such that $\phi(x, y)=0 "$. In fact, given $\theta(x)$, we define

$$
\phi(x, y)=\theta(x)+a(x, y),
$$

where $a(x, y)$ is the (primitive recursive) function with the properties

$$
\begin{aligned}
a(x, y) & =1 & & (y \leqslant x), \\
& =0 & & (y>x) .
\end{aligned}
$$

If on the other hand we are given $\phi(x, y)$ we define $\theta(x)$ by the equations

$$
\begin{gathered}
\theta_{1}(0)=3, \\
\theta_{1}(x+1)=2^{\left(1+\varpi_{2}\left(\theta_{1}(x)\right)\right) \sigma\left(\phi\left(\varpi_{3}\left(\theta_{1}(x)\right)-1, \varpi_{2}\left(\theta_{1}(x)\right)\right)\right)} 3^{\varpi_{3}\left(\theta_{1}(x)\right)+1-\sigma\left(\phi\left(\varpi_{3}\left(\theta_{1}(x)\right)-1, \varpi_{2}\left(\theta_{1}(x)\right)\right)\right)}, \\
\theta(x)=\phi\left(\varpi_{3}\left(\theta_{1}(x)\right)-1, \varpi_{2}\left(\theta_{1}(x)\right)\right),
\end{gathered}
$$

where $\varpi_{r}(x)$ is defined so as to mean "the largest $s$ for which $r^{s}$ divides $x$ ". The function $\sigma(x)$ is defined by the equations $\sigma(0)=0, \sigma(x+1)=1$. It is easily verified that the functions so defined have the desired properties.

We shall now show that questions about the truth of the statements of the form " $\operatorname{does} f(x)$ vanish identically", where $f(x)$ is a computable function, can be reduced to questions about the truth of number-theoretic theorems. It is understood that in each case the rule for the calculation of $f(x)$ is given and that we are satisfied that this rule is valid, i.e. that the machine which should calculate $f(x)$ is circle free (Turing [1], 233). The function $f(x)$, being computable, is general recursive in the Herbrand-Gödel sense, and therefore, by a general theorem due to Kleene $\dagger$, is expressible in the form

$$
\psi(\epsilon y[\phi(x, y)=0]),
$$

where $\epsilon y[\mathfrak{A}(y)]$ means "the least $y$ for which $\mathfrak{A}(y)$ is true" and $\psi(y)$ and $\phi(x, y)$ are primitive recursive functions. Without loss of generality, we may suppose that the functions $\phi, \psi$ take only the values 0,1 . Then, if

† Kleene [3], 727. This result is really superfluous for our purpose, since the proof that every computable function is general recursive proceeds by showing that these functions are of the form (3.2). (Turing [2], 161). 
we define $\rho(x)$ by the equations (3.1) and

$$
\begin{gathered}
\rho(0)=\psi(0)(1-\theta(0)), \\
\rho(x+1)=1-(1-\rho(x)) \sigma\left[1+\theta(x)-\psi\left\{\varpi_{2}\left(\theta_{1}(x)\right)\right\}\right]
\end{gathered}
$$

it will be seen that $f(x)$ vanishes identically if and only if $\rho(x)$ vanishes for infinitely many values of $x$.

The converse of this result is not quite true. We cannot say that the question about the truth of any number-theoretic theorem is reducible to a question about whether a corresponding computable function vanishes identically; we should have rather to say that it is reducible to the problem of whether a certain machine is circle free and calculates an identically vanishing function. But more is true: every number-theoretic theorem is equivalent to the statement that a corresponding machine is circle free. The behaviour of the machine may be described roughly as follows: the machine is one for the calculation of the primitive recursive function $\theta(x)$ of the number-theoretic problem, except that the results of the calculation are first arranged in a form in which the figures 0 and 1 do not occur, and the machine is then modified so that, whenever it has been found that the function vanishes for some value of the argument, then 0 is printed. The machine is circle free if and only if an infinity of these figures are printed, $i$.e. if and only if $\theta(x)$ vanishes for infinitely many values of the argument. That, on the other hand, questions of circle freedom may be reduced to questions of the truth of number-theoretic theorems follows from the fact that $\theta(x)$ is primitive recursive when it is defined to have the value 0 if a certain machine .11 prints 0 or 1 in its $(x+1)$-th complete configuration, and to have the value 1 otherwise.

The conversion calculus provides another normal form for the numbertheoretic theorems, and the one which we shall find the most convenient to use. Every number-theoretic theorem is equivalent to a statement of the form " $\mathbf{A}(\mathbf{n})$ is convertible to 2 for every W.F.F. $\mathbf{n}$ representing a positive integer", A being a W.F.F. determined by the theorem; the property of $\mathbf{A}$ here asserted will be described briefly as " $\mathbf{A}$ is dual". Conversely such statements are reducible to number theoretic theorems. The first half of this assertion follows from our results for computable functions, or directly in this way. Since $\theta(x-1)+2$ is primitive recursive, it is formally definable, say, by means of a formula G. Now there is (Kleene [1], 232) a W.F.F. $\subseteq$ with the property that, if $\mathbf{T}(\mathbf{r})$ is convertible to a formula representing a positive integer for each positive integer $r$, then $\mathscr{P}(\mathbf{T}, \mathbf{n})$ is convertible to $s$, where $s$ is the $n$-th positive integer $t$ (if there is one) for which 
$\mathbf{T}(\mathbf{t})$ conv 2 ; if $\mathbf{T}(\mathbf{t})$ conv 2 for less than $n$ values of $t$ then $\mathcal{P}(\mathbf{T}, \mathbf{n})$ has no normal form. The formula $\mathbf{G}(\boldsymbol{P}(\mathbf{G}, \mathbf{n}))$ is therefore convertible to 2 if and only if $\theta(x)$ vanishes for at least $n$ values of $x$, and is convertible to 2 for every positive integer $x$ if and only if $\theta(x)$ vanishes infinitely often. To prove the second half of the assertion, we take Gödel representations for the formulae of the conversion calculus. Let $c(x)$ be 0 if $x$ is the G.R. of 2 (i.e. if $x$ is $2^{3} \cdot 3^{10} \cdot 5.7^{3} \cdot 11^{28} \cdot 13.17 .19^{10} \cdot 23^{2} .29 .31$. $\left.37^{10} .41^{2} .43 .47^{28} \cdot 53^{2} .59^{2} \cdot 61^{2} \cdot 67^{2}\right)$ and let $c(x)$ be 1 otherwise. Take an enumeration of the G.R. of the formulae into which $\mathbf{A}(\mathbf{m})$ is convertible : let $a(m, n)$ be the $n$-th number in the enumeration. We can arrange the enumeration so that $a(m, n)$ is primitive recursive. Now the statement that $\mathbf{A}(\mathbf{m})$ is convertible to 2 for every positive integer $m$ is equivalent to the statement that, corresponding to each positive integer $m$, there is a positive integer $n$ such that $c(a(m, n))=0$; and this is number-theoretic.

It is easy to show that a number of unsolved problems, such as the problem of the truth of Fermat's last theorem, are number-theoretic. There are, however, also problems of analysis which are number-theoretic. The Riemann hypothesis gives us an example of this. We denote by $\zeta(s)$ the function defined for $\mathfrak{k} s=\sigma>1$ by the series $\sum_{n=1}^{\infty} n^{-s}$ and over the rest of the complex plane with the exception of the point $s=1$ by analytic continuation. The Riemann hypothesis asserts that this function does not vanish in the domain $\sigma>\frac{1}{2}$. It is easily shown that this is equivalent to saying that it does not vanish for $2>\sigma>\frac{1}{2}, \mathfrak{3} s=t>2$, i.e. that it does not vanish inside any rectangle $2>\sigma>\frac{1}{2}+1 / T, T>t>2$, where $T$ is an integer greater than 2. Now the function satisfies the inequalities

$$
\left.\begin{array}{r}
\left|\zeta(s)-\sum_{1}^{N} n^{-s}-\frac{N^{1-s}}{s-1}\right|<2 t(N-2)^{-\frac{t}{t}}, \quad 2<\sigma<\frac{1}{2}, \quad t \geqslant 2, \\
\left|\zeta(s)-\zeta\left(s^{\prime}\right)\right|<60 t\left|s-s^{\prime}\right|, \quad 2<\sigma^{\prime}<\frac{1}{2}, \quad t^{\prime} \geqslant 2,
\end{array}\right\}
$$

and we can define a primitive recursive function $\xi\left(l, l^{\prime}, m, m^{\prime}, N, M\right)$ such that

$$
\left|\xi\left(l, l^{\prime}, m, m^{\prime}, N, M\right)-M\right| \sum_{1}^{N} n^{-s}+\frac{N^{1-s}}{s-1}||<2, \quad\left(s=\frac{l}{l^{\prime}}+i \frac{m}{m^{\prime}}\right),
$$

and therefore, if we put

$$
\xi\left(l, M, m, M, M^{2}+2, M\right)=X(l, m, M),
$$


we have

$$
\left|\zeta\left(\frac{l+\vartheta}{M}+i \frac{m+\vartheta}{M}\right)\right| \geqslant \frac{X(l, m, M)-122 T}{M}
$$

provided that

$$
\begin{aligned}
\frac{1}{2}+\frac{1}{T} \leqslant \frac{l-1}{M}<\frac{l+1}{M}<2-\frac{1}{M}, \quad 2 & <\frac{m-1}{M}<\frac{m+1}{M}<T \\
& \left(-1<\vartheta<1,-1<\vartheta^{\prime}<1\right) .
\end{aligned}
$$

If we define $B\left(M, T^{\prime}\right)$ to be the smallest value of $X(l, m, M)$ for which

$$
\frac{1}{2}+\frac{1}{T}+\frac{1}{M} \leqslant \frac{l}{M}<2-\frac{1}{M}, \quad 2+\frac{1}{M}<\frac{m}{M}<T-\frac{1}{M},
$$

then the Riemann hypothesis is true if for each $T$ there is an $M$ satisfying

$$
B(M, T)>122 T .
$$

If on the other hand there is a $T$ such that, for all $M, B(M, T) \leqslant 122 T$, the Riemann hypothesis is false; for let $l_{M}, m_{M}$ be such that

then

$$
\begin{aligned}
& X\left(l_{M}, m_{M}, M\right) \leqslant 122 T \\
& \left|\zeta\left(\frac{l_{M}+i m_{M}}{M}\right)\right| \leqslant \frac{244 T}{M} .
\end{aligned}
$$

Now if $a$ is a condensation point of the sequence $\left(l_{M}+i m_{M}\right) / M$ then since $\zeta(s)$ is continuous except at $s=1$ we must have $\zeta(a)=0$ implying the falsity of the Riemann hypothesis. Thus we have reduced the problem to the question whether for each $T$ there is an $M$ for which

$$
B(M, T)>122 T .
$$

$B(M, T)$ is primitive recursive, and the problem is therefore numbertheoretic.

4. A type of problem which is not number-theoretic $\dagger$.

Let us suppose that we are supplied with some unspecified means of solving number-theoretic problems; a kind of oracle as it were. We shall 
not go any further into the nature of this oracle apart from saying that it cannot be a machine. With the help of the oracle we could form a new kind of machine (call them $o$-machines), having as one of its fundamental processes that of solving a given number-theoretic problem. More definitely these machines are to behave in this way. The moves of the machine are determined as usual by a table except in the case of moves from a certain internal configuration $\mathfrak{b}$. If the machine is in the internal. configuration $\mathfrak{o}$ and if the sequence of symbols marked with $l$ is then the well-formed $\dagger$ formula $\mathbf{A}$, then the machine goes into the internal configuration $p$ or $\mathbf{t}$ according as it is or is not true that $\mathbf{A}$ is dual. The decision as to which is the case is referred to the oracle.

These machines may be described by tables of the same kind as those used for the description of $a$-machines, there being no entries, however, for the internal configuration $\mathfrak{b}$. We obtain description numbers from thesc tables in the same way as before. If we make the convention that, in. assigning numbers to internal configurations, $\mathfrak{v}, \mathfrak{p}, \mathrm{t}$ are always to be $q_{2}, q_{3}, q_{4}$, then the description numbers determine the behaviour of the machines uniquely.

Given any one of these machines we may ask ourselves the question whether or not it prints an infinity of figures 0 or 1 ; I assert that this class of problem is not number-theoretic. In view of the definition of "number theoretic problem" this means that it is not possible to construct an $o$-machine which, when supplied $\ddagger$ with the description of any other $o$-machine, will determine whether that machine is $o$-circle free. The argument may be taken over directly from Turing [1], $\S 8$. We say that a number is $o$-satisfactory if it is the description number of an $o$-circle free machine. Then, if there is an o-machine which will determine of any integer whether it is $o$-satisfactory, there is also an $o$-machine to calculate the values of the function $1-\phi_{n}(n)$. Let $r(n)$ be the $n$-th $o$-satisfactory number and let $\phi_{n}(m)$ be the $m$-th figure printed by the $o$-machine whose description number is $r(n)$. This $o$-machine is circle free and there is therefore an 0 -satisfactory number $K$ such that $\phi_{K}(n)=1-\phi_{n}(n)$ for all $n$. Putting $n=K$ yields a contradiction. This completes the proof that problems of circle freedom of $o$-machines are not number-theoretic.

Propositions of the form that an $o$-machine is $o$-circle free can always be put in the form of propositions obtained from formulae of the functional calculus of the first order by replacing some of the functional variables by primitive recursive relations. Compare foot-note $\dagger$ on page 168 .

$\dagger$ Without real loss of generality we may suppose that $\mathbf{A}$ is always well formed.

† Compare Turing [1], §6, 7 . 


\section{Syntactical theorems as number-theoretic theorems.}

I now mention a property of number-theoretic theorems which suggests that there is reason for regarding them as of particular importance.

Suppose that we have some axiomatic system of a purely formal nature. We do not concern ourselves at all in interpretations for the formulae of this system; they are to be regarded as of interest for themselves. An example of what is in mind is afforded by the conversion calculus $(\S 1)$. Every sequence of symbols "A conv B", where A and B are well formed formulae, is a formula of the axiomatic system and is provable if the W.F.F. A is convertible to $\mathbf{B}$. The rules of conversion give us the rules of procedure in this axiomatic system.

Now consider a new rule of procedure which is reputed to yield only formulae provable in the original sense. We may ask ourselves whether such a rule is valid. The statement that such a rule is valid would be number-theoretic. To prove this, let us take Gödel representations for the formulae, and an enumeration of the provable formulae; let $\phi(r)$ be the G.R. of the $r$-th formula in the enumeration. We may suppose $\phi(r)$ to be primitive recursive if we are prepared to allow repetitions in the enumeration. Let $\psi(r)$ be the G.R. of the $r$-th formula obtained by the new rule, then the statement that this new rule is valid is equivalent to the assertion of

$$
(r)(\exists s)[\psi(r)=\phi(s)]
$$

(the domain of individuals being the natural numbers). It has been shown in $\S 3$ that such statements are number-theoretic.

It might plausibly be argued that all those theorems of mathematics which have any significance when taken alone are in effect syntactical theorems of this kind, stating the validity of certain "derived rules" of procedure. Without going so far as this, I should assert that theorems of this kind have an importance which makes it worth while to give them special consideration.

\section{Logic formulae.}

We shall call a formula $\mathbf{L}$ a logic formula (or, if it is clear that we are speaking of a W.F.F., simply a logic) if it has the property that, if $\mathbf{A}$ is a formula such that $\mathbf{L}(\mathbf{A})$ conv 2 , then $\mathbf{A}$ is dual.

A logic formula gives us a means of satisfying ourselves of the truth of number-theoretic theorems. For to each number-theoretic proposition there corresponds a W.F.F. A which is dual if and only if the proposition is true. Now, if $\mathbf{L}$ is a logic and $\mathbf{L}(\mathbf{A})$ conv 2 , then $\mathbf{A}$ is dual and we know that 
the corresponding number-theoretic proposition is true. It does not follow that, if $L$ is a logic, we can use $L$ to satisfy ourselves of the truth of any number-theoretic theorem.

If $\mathbf{L}$ is a logic, the set of formulae $A$ for which $L(A)$ conv 2 will be called the extent of $\mathrm{L}$.

It may be proved by the use of (D), (E), p. 166, that there is a formula $X$ such that, if $\mathbf{M}$ has a normal form, has no free variables and is not convertible to 2, then $X(\mathbf{M})$ conv 1, but, if $\mathbf{M}$ conv 2, then $X(\mathbf{M})$ conv 2. If $\mathrm{L}$ is a logic, then $\lambda x . X(\mathbf{L}(x))$ is also a logic whose extent is the same as that of $\mathbf{L}$, and which has the property that, if $\mathrm{A}$ has no free variables, then

$$
\{\lambda x \cdot X(\mathbf{L}(x))\}(\mathbf{A})
$$

either is always convertible to 1 or to 2 or else has no normal form. A logic with this property will be said to be standardized.

We shall say that a logic $\mathrm{L}^{\prime}$ is at least as complete as a logic $\mathrm{L}$ if the extent of $\mathbf{L}$ is a subset of the extent of $\mathbf{L}^{\prime}$. The logic $\mathbf{L}^{\prime}$ is more complete than $\mathbf{L}$ if the extent of $\mathbf{L}$ is a proper subset of the extent of $\mathbf{L}^{\prime}$.

Suppose that we have an effective set of rules by which we can prove formulae to be dual; i.e. we have a system of symbolic logic in which the propositions proved are of the form that certain formulae are dual. Then we can find a logic formula whose extent consists of just those formulae which can be proved to be dual by the rules; that is to say, there is a rule for obtaining the logic formula from the system of symbolic logic. In fact the system of symbolic logic enables us to obtain $\dagger$ a computable function of positive integers whose values run through the Gödel representations of the formulae provable by means of the given rules. By the theorem of equivalence of computable and $\lambda$-definable functions, there is a formula $\mathbf{J}$ such that $\mathbf{J}(1), \mathbf{J}(2), \ldots$ are the $\mathrm{G} . \mathrm{R}$. of these formulae. Now let

$$
W \rightarrow \lambda j v . \oplus(\lambda u . \delta(j(u), v), 1, I, 2) .
$$

Then $I$ assert that $W(\mathbf{J})$ is a logic with the required properties. The properties of $\Phi$ imply that $\Phi(\mathbf{C}, 1)$ is convertible to the least positive integer $\mathbf{n}$ for which $\mathbf{C}(\mathbf{n})$ conv 2 , and has no normal form if there is no such integer. Consequently $\mathscr{P}(\mathbf{C}, 1, I, 2)$ is convertible to 2 if $\mathbf{C}(\mathbf{n})$ conv 2 for some positive integer $n$, and it has no normal form otherwise. That is to say that $W(\mathbf{J}, \mathbf{A})$ conv 2 if and only if $\delta(\mathbf{J}(\mathbf{n}), \mathbf{A})$ conv 2 , some $n$, i.e. if $\mathbf{J}(\mathbf{n})$ conv $\mathbf{A}$ some $n$. 
There is conversely a formula $W^{\prime}$ such that, if $\mathrm{L}$ is a logic, then $W^{\prime}(\mathrm{L})$ enumerates the extent of $\mathbf{L}$. For there is a formula $Q$ such that $Q(\mathbf{L}, \mathbf{A}, \mathbf{n})$ conv 2 if and only if $\mathbf{L}(\mathbf{A})$ is convertible to 2 in less than $n$ steps. We then put

$$
W^{\prime} \rightarrow \lambda l n . \text { form }(\varpi(2, \mathscr{P}(\lambda x \cdot Q(l, \text { form }(\varpi(2, x)), \varpi(3, x)), n))) .
$$

Of course, $W^{\prime}(W(\mathbf{J}))$ normally entirely different from $\mathbf{J}$ and $W\left(W^{\prime}(\mathbf{L})\right)$ from $L$.

In the case where we have a symbolic logic whose propositions can be interpreted as number-theoretic theorems, but are not expressed in the form of the duality of formulac, we shall again have a corresponding logic formula, but its relation to the symbolic logic is not so simple. As an example let us take the case where the symbolic logic proves that certain primitive recursive functions vanish infinitely often. As was shown in $\S 3$, we can associate with each such proposition a W.F.F. which is dual if and only if the proposition is true. When we replace the propositions of the symbolic logic by theorems on the duality of formulae in this way, our previous argument applies and we obtain a certain logic formula $\mathbf{L}$. However, $L$ does not determine uniquely which are the propositions provable in the symbolic logic; for it is possible that " $\theta_{1}(x)$ vanishes infinitely often" and " $\theta_{2}(x)$ vanishes infinitely often" are both associated with "A is dual", and that the first of these propositions is provable in the system, but the second not. However, if we suppose that the system of symbolic logic is sufficiently powerful to be able to carry out the argument on pp. 170-171 then this difficulty cannot arise. There is also the possibility that there may be formulae in the extent of $L$ with no propositions of the form " $\theta(x)$ vanishes infinitely often" corresponding to them. But to each such formula we can assign (by a different argument) a proposition $p$ of the symbolic logic which is a necessary and sufficient condition for $\mathbf{A}$ to be dual. With $p$ is associated (in the first way) a formula $\mathbf{A}^{\prime}$. Now $\mathbf{L}$ can always be modified so that its extent contains $\mathbf{A}^{\prime}$ whenever it contains A.

We shall be interested principally in questions of completeness. Let us suppose that we have a class of systems of symbolic logic, the propositions of these systems being expressed in a uniform notation and interpretable as number-theoretic theorems; suppose also that there is a rule by which we can assign to each proposition $p$ of the notation a W.F.F. $\mathbf{A}_{p}$ which is dual if and only if $p$ is true, and that to each W.F.F. A we can assign a propo- 
sition $p_{\mathbf{A}}$ which is a necessary and sufficient condition for $\mathbf{A}$ to be dual. $p_{\mathrm{A}}$, is to be expected to differ from $p$. To each symbolic logic $C$ we can assign two logic formulae $\mathrm{L}_{C}$ and $\mathbf{L}_{C}{ }^{\prime}$. A formula $A$ belongs to the extent of $\mathbf{L}_{C}$ if $p_{\mathbf{A}}$ is provable in $C$, while the extent of $\mathbf{L}_{C}{ }^{\prime}$ consists of all $\mathbf{A}_{p}$ : where $p$ is provable in $C$. Let us say that the class of symbolic logics is complete if each true proposition is provable in one of them: let us also say that a class of logic formulae is complete if the set-theoretic sum of the extents of these logics includes all dual formulae. I assert that a necessary condition for a class of symbolic logics $C$ to be complete is that the class of logics $\mathbf{L}_{C}$ is complete, while a sufficient condition is that the class of logics $\mathbf{L}_{C}{ }^{\prime}$ is complete. Let us suppose that the class of symbolic logics is complete; consider $p_{\mathbf{A}}$, where $\mathbf{A}$ is arbitrary but dual. It must be provable in one of the systems, $C$ say. A therefore belongs to the extent of $\mathrm{L}_{C}$, i.e. the class of logics $\mathbf{L}_{C}$ is complete. Now suppose the class of logics $\mathbf{L}_{C}{ }^{\prime}$ to be complete. Let $p$ be an arbitrary true proposition of the notation; $\mathbf{A}_{p}$ must belong to the extent of some $\mathbf{L}_{C}{ }^{\prime}$, and this means that $p$ is provable in $C$.

We shall say that a single logic formula $\mathrm{L}$ is complete if its extent includes all dual formulae; that is to say, it is complete if it enables us to prove every true number-theoretic theorem. It is a consequence of the theorem of Gödel (if suitably extended) that no logic formula is complete, and this also follows from $(C)$, p. 165, or from the results of Turing [1], §8, when taken in conjunction with $\S 3$ of the present paper. The idea of completeness of a logic formula is not therefore very important, although it is useful to have a term for it.

Suppose $\mathbf{Y}$ to be a W.F.F. such that $\mathbf{Y}(\mathbf{n})$ is a logic for each positive integer $n$. The formulae of the extent of $\mathbf{Y}(\mathbf{n})$ are enumerated by $W(\mathbf{Y}(\mathbf{n}))$, and the combined extents of these logics by

$$
\lambda r . W(\mathbf{Y}(\varpi(2, v), \varpi(3, r))) .
$$

If we put

$$
\Gamma \rightarrow \lambda y \cdot W^{\prime}(\lambda r . W(y(\varpi(2, r), \varpi(3, r)))),
$$

then $\Gamma(\mathbf{Y})$ is a logic whose extent is the combined extent of

$$
\mathbf{Y}(1), \quad \mathbf{Y}(2), \quad \mathbf{Y}(3), \ldots .
$$

To each W.F.F. L we can assign a W.F.F. $V($ L $)$ such that a necessary and sufficient condition for $\mathbf{L}$ to be a logic formula is that $V(\mathbf{L})$ is dual. Let $\mathrm{Nm}$ be a W.F.F. which enumerates all formulae with normal forms 
and no free variables. Then the condition for $\mathrm{L}$ to be a logic is that $\mathbf{L}(\mathrm{Nm}(\mathbf{r}), \mathbf{s})$ conv 2 for all positive integers $r$, s, i.e. that

$$
\lambda a . \mathrm{L}(\mathrm{Nm}(\varpi(2, a)), \varpi(3, a))
$$

is dual. We may therefore put

$$
V \rightarrow \lambda l a . l(\operatorname{Nm}(\varpi(2, a)), \varpi(3, a)) .
$$

\section{Ordinals.}

We begin our treatment of ordinals with some brief definitions from the C'antor theory of ordinals, but for the understanding of some of the proofs a greater amount of the Cantor theory is necessary than is set out here.

Suppose that we have a class determined by the propositional function $D(x)$ and a relation $G(x, y)$ ordering its members, i.e. satisfying

$$
\begin{aligned}
& G(x, y) \& G(y, z) \supset G(x, z), \\
& D(x) \& D(y) \supset G(x, y) \vee G(y, x) \vee x=y \\
& G(x, y) \supset D(x) \& D(y) \\
& \sim G(x, x)
\end{aligned}
$$

The class defined by $D(x)$ is then called a series with the ordering relation $G^{\prime}(x, y)$. The series is said to be well ordered and the ordering relation is called an ordinal if every sub-series which is not void has a first term, i.e. if

$$
\begin{aligned}
&\left(D^{\prime}\right)\left\{(\exists x)\left(D^{\prime}(x)\right) \&(x)\right.\left(D^{\prime}(x) \supset D(x)\right) \\
&\left.\supset(\exists z)(y)\left[D^{\prime}(z) \&\left(D^{\prime}(y) \supset G(z, y) \mathbf{v} z=y\right)\right]\right\} .
\end{aligned}
$$

'I'he condition (7.2) is equivalent to another, more suitable for our purposes, namely the condition that every descending subsequence must terminate; formally

$$
(x)\left\{D^{\prime}(x) \supset D(x) \&(\exists y)\left(D^{\prime}(y) \& G(y, x)\right)\right\} \supset(x)\left(\sim D^{\prime}(x)\right) .
$$

The ordering relation $G(x, y)$ is said to be similar to $G^{\prime}(x, y)$ if there is a one-one correspondence between the series transforming the one relation 
into the other. This is best expressed formally, thus

$$
\begin{aligned}
(\exists M)[(x)\{D(x) & \left.\supset\left(\exists x^{\prime}\right) M\left(x, x^{\prime}\right)\right\} \&\left(x^{\prime}\right)\left\{D^{\prime}\left(x^{\prime}\right) \supset(\exists x) M\left(x, x^{\prime}\right)\right\} \\
& \&\left\{\left(M\left(x, x^{\prime}\right) \& M\left(x, x^{\prime \prime}\right)\right) \mathrm{v}\left(M\left(x^{\prime}, x\right) \& M\left(x^{\prime \prime}, x\right)\right) \supset x^{\prime}=x^{\prime \prime}\right\} \\
& \left.\&\left\{M\left(x, x^{\prime}\right) \& M\left(y, y^{\prime}\right) \supset\left(G(x, y) \equiv G\left(x^{\prime}, y^{\prime}\right)\right)\right\}\right] .
\end{aligned}
$$

Ordering relations are regarded as belonging to the same ordinal if and only if they are similar.

We wish to give names to all the ordinals, but this will not be possible until they have been restricted in some way; the class of ordinals, as at present defined, is more than enumerable. The restrictions that we actually impose are these : $D(x)$ is to imply that $x$ is a positive integer; $D(x)$ and $G(x, y)$ are to be computable properties. Both of the propusitional functions $D(x), G(x, y)$ can then be described by mears of a single W.F.F. $\Omega$ with the properties:

$\boldsymbol{\Omega}(\mathbf{m}, \mathbf{n})$ conv 4 unless both $D(m)$ and $D(n)$ are true,

$\Omega(\mathbf{m}, \mathbf{m})$ conv 3 if $D(m)$ is true,

$\mathbf{\Omega}(\mathbf{m}, \mathbf{n})$ conv 2 if $D(m), D(n), G(m, n), \sim(m=n)$ are true,

$\boldsymbol{\Omega}(\mathbf{m}, \mathbf{n})$ conv $\mathbf{l}$ if $D(m), D(n), \sim G(m, n), \sim(m=n)$ are true.

In consequence of the conditions to which $D(x), G(x, y)$ are subjected, $\Omega$ must further satisfy:

(a) if $\boldsymbol{\Omega}(\mathbf{m}, \mathbf{n})$ is convertible to $\mathbf{l}$ or 2 , then $\boldsymbol{\Omega}(\mathbf{m}, \mathbf{m})$ and $\boldsymbol{\Omega}(\mathbf{n}, \mathbf{n})$ are convertible to 3 ,

(b) if $\Omega(\mathbf{m}, \mathbf{m})$ and $\Omega(\mathbf{n}, \mathbf{n})$ are convertible to 3 , then $\Omega(\mathbf{m}, \mathbf{n})$ is convertible to 1,2 , or 3 ,

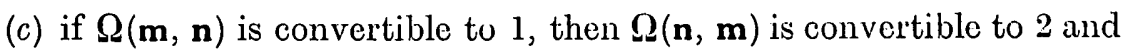
conversely,

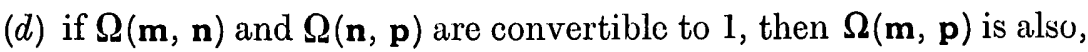

(e) there is no sequence $m_{1}, m_{2}, \ldots$ such that $\Omega\left(\mathbf{m}_{i+1}, \mathbf{m}_{i}\right)$ conv 2 for each positive integer $i$,

(f) $\Omega(\mathbf{m}, \mathbf{n})$ is always convertible to $1,2,3$, or 4 .

If a formula $\Omega$ satisfies these conditions then there are corresponding propositional functions $D(x), G(x, y)$. We shall therefore say that $\boldsymbol{\Omega}$ is 
an ordinal formula if it satisfies the conditions $(a)-(f)$. It will be seen that a consequence of this definition is that $\mathrm{Dt}$ is an ordinal formula ; it represents the ordinal $\omega$. The definition that we have given does not pretend to have virtues such as elegance or convenience. It has been introduced rather to fix our ideas and to show how it is possible in principle to describe ordinals by means of well formed formulae. The definitions could be modified in a number of ways. Some such modifications are quite trivial; they are typified by modifications such as changing the numbers $1,2,3,4$, used in the definition, to others. Two such definitions will be said to be equivalent; in general, we shall say that two definitions are equivalent if there are W.F.F. T, $\mathbf{T}^{\prime}$ such that, if $\mathbf{A}$ is an ordinal formula under one definition and represents the ordinal $a$, then $\mathbf{T}^{\prime}(\mathbf{A})$ is an ordinal formula under the second definition and represents the same ordinal; and, conversely, if $\mathbf{A}^{\prime}$ is an ordinal formula under the second definition representing $a$, then $\mathbf{T}\left(\mathbf{A}^{\prime}\right)$ represents $\alpha$ under the first definition. Besides definitions equivalent in this sense to our original definition, there are a number of other possibilities upen. Suppose for instance that we do not require $D(x)$ and $G(x, y)$ to be computable, but that we require only that $D(x)$ and $G(x, y) \& x<y$ are axiomatic $t$. This leads to a definition of an ordinal formula which is (presumably) not equivalent to the definition that we are using $\ddagger$. There are numerous possibilities, and little to guide us in choosing one definition rather than another. No one of them could well be described as "wrong"; some of them may be found more valuable in applications than others, and the particular choice that we have made has been determined partly by the applications that we have in view. In the case of theorems of a negative character, one would wish to prove them for each one of the possible definitions of "ordinal formula". 'This programme could, I think, be carried through for the negative results of $\S 9,10$.

Before leaving the subject of possible ways of defining ordinal formulae, I must mention another definition due to Church and Kleene (Church and Kleene [1]). We can make use of this definition in constructing ordinal logics, but it is more convenicnt to use a slightly different definition which is equivalent (in the sense just described) to the Church-Kleene definition as modified in Church [4].

f To require $G(x, y)$ to be axionatic amounts to requiring $G(x, y)$ to be computable on account of (7. 1) (ii).

$\ddagger$ On the other hand, if $\nu(x)$ is axiomatic and $C_{i}(x, y)$ is computable in the modified sense that there is a rule for determining whether $G(x, y)$ is true which leads to a definite result in all cases where $D(x)$ and $D(y)$ are true, the corresponding definition of ordinal formula is equivalent to our definition. 'Tu give the proof would be tou much of a digression. Probably other equivalences of this kind hold. 
Introduce the abbreviations

$$
\begin{aligned}
& U \rightarrow \lambda u f x \cdot u(\lambda y \cdot f(y(I, x))), \\
& \text { Suc } \rightarrow \lambda a u f x \cdot f(a(u, f, x)) .
\end{aligned}
$$

We define first a partial ordering relation " $<$ " which holds between certain pairs of W.F.F. [conditions (1)-(5)].

(1) If $\mathbf{A}$ conv $\mathbf{B}$, then $\mathbf{A}<\mathbf{C}$ implies $\mathbf{B}<\mathbf{C}$ and $\mathbf{C}<\mathbf{A}$ implies $\mathbf{C}<\mathbf{B}$.

(2) $\mathbf{A}<\operatorname{Suc}(\mathbf{A})$.

(3) For any positive integers $m$ and $n, \lambda u f x . \mathbf{R}(\mathbf{n})<\lambda u f x . \mathbf{R}(\mathbf{m})$ implies $\lambda u f x . \mathbf{R}(\mathbf{n})<\lambda u f x . u(\mathbf{R})$.

(4) If $A<B$ and $B<C$, then $A<C$. (1)-(4) are required for any W.F.F. A, B, C, $\lambda u f x$. R.

(5) The relation $\mathbf{A}<\mathbf{B}$ holds only when compelled to do so by (1)-(4).

We define C-K ordinal formulae by the conditions (6)-(10).

(6) If $\mathbf{A}$ conv $\mathbf{B}$ and $\mathbf{A}$ is a $\mathrm{C}-\mathrm{K}$ ordinal formula, then $\mathbf{B}$ is a $\mathrm{C}-\mathrm{K}$ ordinal formula.

(7) $U$ is a C-K ordinal formula.

(8) If $\mathbf{A}$ is a $\mathbf{C}-\mathrm{K}$ ordinal formula, then $\operatorname{Suc}(\mathbf{A})$ is a $\mathrm{C}-\mathrm{K}$ ordinal formula.

(9) If $\lambda u f x \cdot \mathbf{R}(\mathbf{n})$ is a C-K ordinal formula and

$$
\lambda u f x \cdot \mathbf{R}(\mathbf{n})<\lambda u f x \cdot \mathbf{R}(S(\mathbf{n}))
$$

for each positive integer $n$, then $\lambda u f x . u(\mathbf{R})$ is a C-K ordinal formula $\dagger$.

(10) A formula is a C-K ordinal formula only if compelled to be so by (6)-(9).

$\dagger$ If we also allow $\lambda u f x . u(\mathbf{R})$ to be a C-K ordinal formula when

$$
\lambda u f x . \mathbf{n}(\mathbf{R}) \operatorname{conv} \lambda u f x . S(\mathbf{n}, \mathbf{R})
$$

for all $n$, then the formulae for sum, product and exponentiation of C-K ordinal formulae can be much simplified. For instance, if $\mathbf{A}$ and $\mathbf{B}$ represent $\alpha$ and $\beta$, then

$$
\lambda u f x, \mathbf{B}(u, f, \mathbf{A}(u, f, x))
$$

represents $\alpha+\beta$. Property (6) remains true. 
The representation of ordinals by formulae is described by (11)-(15).

(11) If $\mathbf{A}$ conv $\mathbf{B}$ and $\mathbf{A}$ represents $a$, then $\mathbf{B}$ represents $a$.

(12) $U$ represents 1.

(13) If $\mathbf{A}$ represents $\alpha$, then Suc (A) represents $a+1$.

(14) If $\lambda u f x \cdot \mathbf{R}(\mathbf{n})$ represents $a_{n}$ for each positive integer $n$, then $\lambda u f x . u(\mathbf{R})$ represents the upper bound of the sequence $a_{1}, a_{2}, a_{3}, \ldots$.

(15) A formula represents an ordinal only when compelled to do so by (11)-(14).

We denote any ordinal represented by $\mathbf{A}$ by $\boldsymbol{\Xi}_{\mathbf{A}}$ without prejudice to the possibility that more than one ordinal may be represented by $\mathbf{A}$. We shall write $\mathbf{A} \leqslant \mathbf{B}$ to mean $\mathbf{A}<\mathbf{B}$ or $\mathbf{A}$ conv $\mathbf{B}$.

In proving properties of $\mathrm{C}-\mathrm{K}$ ordinal formulae we shall often use a kind of analogue of the principle of transfinite induction. If $\phi$ is some property and we have:

(a) If $\mathbf{A}$ conv $\mathbf{B}$ and $\phi(\mathbf{A})$, then $\phi(\mathbf{B})$,

(b) $\phi(U)$,

(c) If $\phi(\mathbf{A})$, then $\phi(\operatorname{Suc}(\mathbf{A}))$,

(d) If $\phi(\lambda u f x . \mathbf{R}(\mathbf{n}))$ and $\lambda u f x \cdot \mathbf{R}(\mathbf{n})<\lambda u f x \cdot \mathbf{R}(S(\mathbf{n}))$ for each positive integer $n$, then

$$
\phi(\lambda u f x . u(\mathbf{R}))
$$

then $\phi(\mathbf{A})$ for each $\mathrm{C}-\mathrm{K}$ ordinal formula $\mathbf{A}$. To prove the validity of this principle we have only to observe that the class of formulae $\mathbf{A}$ satisfying $\phi(\mathbf{A})$ is one of those of which the class of C-K ordinal formulae was defined to be the smallest. We can use this principle to help us to prove :-

(i) Every C-K ordinal formula is convertible to the form $\lambda u f x . \mathbf{B}$, where $\mathbf{B}$ is in normal form.

(ii) There is a method by which for any C-K ordinal formula, we can determine into which of the forms $U$, Suc $(\lambda u f x . \mathbf{B}), \lambda u f x . u(\mathbf{R})$ (where $u$ is free in $\mathbf{R}$ ) it is convertible, and by which we can determine $\mathbf{B}, \mathbf{R}$. In each case $\mathbf{B}, \mathbf{R}$ are unique apart from conversions.

(iii) If $\mathbf{A}$ represents any ordinal, $\Xi_{A}$ is unique. If $\Xi_{A}, \Xi_{B}$ exist and $\mathbf{A}<\mathbf{B}$, then $\Xi_{A}<\Xi_{B}$. 
(iv) If $\mathbf{A}, \mathbf{B}, \mathbf{C}$ are $\mathbf{C}-\mathrm{K}$ ordinal formulae and $\mathbf{B}<\mathbf{A}, \mathbf{C}<\mathbf{A}$, then either $\mathbf{B}<\mathbf{C}, \mathbf{C}<\mathbf{B}$, or $\mathbf{B} \operatorname{conv} \mathbf{C}$.

(v) A formula $\mathbf{A}$ is a $\mathrm{C}-\mathrm{K}$ ordinal formula if:

(A) $U \leqslant \mathbf{A}$,

(B) If $\lambda u f x . u(\mathbf{R}) \leqslant \mathbf{A}$ and $n$ is a positive integer, then

$$
\lambda u f x \cdot \mathbf{R}(\mathbf{n})<\lambda u f x \cdot \mathbf{R}(S(\mathbf{n})),
$$

(C) For any two W.F.F. B, C with $\mathbf{B}<\mathbf{A}, \mathbf{C}<\mathbf{A}$ we have $\mathbf{B}<\mathbf{C}, \mathbf{C}<\mathbf{B}$, or $\mathbf{B}$ conv $\mathbf{C}$, but never $\mathbf{B}<\mathbf{B}$,

(D) There is no infinite sequence $\mathbf{B}_{1}, \mathbf{B}_{2}, \ldots$ for which

$$
\mathbf{B}_{\mathbf{r}}<\mathbf{B}_{\mathbf{r}-1}<\mathbf{A}
$$

for each $r$.

(vi) There is a formula $H$ such that, if $\mathbf{A}$ is a $\mathrm{C}-\mathrm{K}$ ordinal formula, then $H(\mathrm{~A})$ is an ordinal formula representing the same ordinal. $H(\mathrm{~A})$ is not an ordinal formula unless $\mathbf{A}$ is a $\mathrm{C}-\mathrm{K}$ ordinal formula.

Proof of (i). Take $\phi(\mathbf{A})$ to be " $\mathbf{A}$ is convertible to the form $\lambda u f x$. B, where B is in normal form". The conditions $(a)$ and $(b)$ are trivial. For (c), suppose that $\mathbf{A}$ conv $\lambda u f x$. B, where $\mathbf{B}$ is in normal form; then.

$$
\operatorname{Suc}(\mathbf{A}) \operatorname{conv} \lambda u f x . f(\mathbf{B})
$$

and $f(\mathbf{B})$ is in normal form. For $(d)$ we have only to show that $u(\mathbf{R})$ has $a$ normal form, i.e. that $\mathbf{R}$ has a normal form; and this is true since $\mathbf{R}(1)$ has a normal form.

Proof of (ii). Since, by hypothesis, the formula is a C-K ordinal formula we have only to perform conversions on it until it is in one of the forms described. It is not possible to convert it into two of these three forms. For suppose that $\lambda u f x . f(\mathbf{A}(u, f, x)) \operatorname{conv} \lambda u f x . u(\mathbf{R})$ and is a C-K ordinal formula; it is then convertible to the form $\lambda u f x$. $\mathbf{B}$, where $\mathbf{B}$ is in normal form. But the normal form of $\lambda u f x . u(\mathbf{R})$ can be obtained by conversions on $\mathbf{R}$, and that of $\lambda u f x . f(\mathbf{A}(u, f, x))$ by conversions on $\mathbf{A}(u, f, x)$ (as follows from Church and Rosser [1], Theorem 2); this, however, would imply that the formula in question had two normal forms, one of form $\lambda u f x . u(\mathbf{S})$ and one of form $\lambda u f x . f(\mathbf{C})$, which is impossible. Or let $U \operatorname{conv} \lambda u f x . u(\mathbf{R})$, where $\mathbf{R}$ is a well formed formula with $u$ as a free variable. We may suppose $\mathbf{R}$ to be in normal form. Now $U$ is $\lambda u f x . u(\lambda y . f(y(I, x)))$. By 
(A), p. $165, \mathbf{R}$ is identical with $\lambda y \cdot f(y(I, x))$, which does not have $u$ as a free variable. It now remains to show only that if

$\operatorname{Suc}(\lambda u f x . \mathbf{B})$ conv Suc $\left(\lambda u f x . \mathbf{B}^{\prime}\right)$ and $\lambda u f x . u(\mathbf{R}) \operatorname{conv} \lambda u f x . u\left(\mathbf{R}^{\prime}\right)$, then $\mathbf{B}$ conv $\mathbf{B}^{\prime}$ and $\mathbf{R}$ conv $\mathbf{R}^{\prime}$.

If

then
$\operatorname{Suc}\left(\lambda u f x\right.$. B) conv Suc $\left(\lambda u f x . \mathbf{B}^{\prime}\right)$,

$$
\lambda u f x . f(\mathbf{B}) \operatorname{conv} \lambda u f x . f\left(\mathbf{B}^{\prime}\right) \text {; }
$$

but both of these formulae can be brought to normal form by conversions on $\mathbf{B}, \mathbf{B}^{\prime}$ and therefore $\mathbf{B}$ conv $\mathbf{B}^{\prime}$. The same argument applies in the case in which $\lambda u f x . u(\mathbf{R}) \operatorname{conv} \lambda u f x . u\left(\mathbf{R}^{\prime}\right)$.

Proof of (iii). To prove the first half, take $\phi(\mathrm{A})$ to be " $\Xi_{\mathrm{A}}$ is unique". Then (7.5) (a) is trivial, and (b) follows from the fact that $U$ is not convertible either to the form Suc (A) or to $\lambda u f x . u(\mathbf{R})$, where $\mathbf{R}$ has $u$ as a free variable. For $(c)$ : Suc (A) is not convertible to the form $\lambda u f x . u(\mathbf{R})$; the possibility that Suc (A) represents an ordinal on account of (12) or (14) is therefore eliminated. By (13), Suc (A) represents $a^{\prime}+1$ if $\mathbf{A}^{\prime}$ represents $a^{\prime \prime}$ and $\operatorname{Suc}(\mathbf{A})$ conv Suc $\left(\mathbf{A}^{\prime}\right)$. If we suppose that $\mathbf{A}$ represents $a$, then $\mathbf{A}, \mathbf{A}^{\prime}$, being C-K ordinal formulae, are convertible to the forms $\lambda u f x . \mathbf{B}, \lambda u f x . \mathbf{B}^{\prime}$; but then, by (ii), $\mathbf{B}$ conv $\mathbf{B}^{\prime}$, i.e. $\mathbf{A}$ conv $\mathbf{A}^{\prime}$, and therefore $\alpha=\alpha^{\prime}$ by the hypothesis $\phi(\mathbf{A})$. Then $\Xi_{\text {Suc (A) }}=\alpha^{\prime}+1$ is unique. For $(d): \lambda u f x . u(\mathbf{R})$ is not convertible to the form $\operatorname{Suc}(\mathbf{A})$ or to $U$ if $\mathbf{R}$ has $u$ as a free variable. If $\lambda u f x . u(\mathbf{R})$ represents an ordinal, it is so therefore in virtue of (14), possibly together with (11). Now, if $\lambda u f x . u(\mathbf{R})$ conv $\lambda u f x . u\left(\mathbf{R}^{\prime}\right)$, then $\mathbf{R}$ conv $\mathbf{R}^{\prime}$, so that the sequence $\lambda u f x . \mathbf{R}(1), \lambda u f x, \mathbf{R}(2), \ldots$ in (14) is unique apart from conversions. Then, by the induction hypothesis, the sequence $a_{1}, a_{2}, a_{3}, \ldots$ is unique. The only ordinal that is represented by $\lambda u f x . u(\mathrm{R})$ is the upper bound of this sequence; and this is unique.

For the second half we use a type of argument rather different from our transfinite induction principle. The formulae $\mathbf{B}$ for which $\mathbf{A}<\mathbf{B}$ form the smallest class for which:

Suc (A) belongs to the class.

If $\mathbf{C}$ belongs to the class, then Suc (C) belongs to it.

If $\lambda u f x \cdot \mathbf{R}(\mathbf{n})$ belongs to the class and

$$
\lambda u f x . \mathbf{R}(\mathbf{n})<\lambda u f x . \mathbf{R}(\mathbf{m}),
$$

where $m, n$ are some positive integers, then $\lambda u f x . u(\mathbf{R})$ belongs to it.

If $\mathbf{C}$ belongs to the class and $\mathbf{C}$ conv $\mathbf{C}^{\prime}$, then $\mathbf{C}^{\prime}$ belongs to it. 
It will be sufficient to prove that the class of formulae B for which either $\Xi_{B}$ does not exist or $\Xi_{A}<\Xi_{B}$ satisfies the conditions (7.6). Now

$$
\begin{gathered}
\Xi_{\text {Suc (A) }}=\Xi_{A}+1>\Xi_{A}, \\
\Xi_{\text {Suc (C) }}>\Xi_{C}>\Xi_{A} \text { if } C \text { is in the class. }
\end{gathered}
$$

If $\Xi_{\lambda u f x . \mathrm{R}(\mathrm{n})}$ does not exist, then $\Xi_{\lambda u f x . u(\mathrm{R})}$ does not exist, and therefore $\lambda u f x . u(\mathbf{R})$ is in the class. If $\Xi_{\lambda u f x . \mathbf{R}(\mathbf{n})}$ exists and is greater than $\Xi_{\mathbf{A}}$, and $\lambda u f x . \mathbf{R}(\mathbf{n})<\lambda u f x . \mathbf{R}(\mathbf{m})$, then

$$
\Xi_{\lambda u f x \cdot u(\mathrm{R})} \geqslant \Xi_{\lambda u f x \cdot \mathrm{R}(\mathrm{n})}>\Xi_{\mathrm{A}},
$$

so that $\lambda u f x . u(\mathbf{R})$ belongs to the class.

Proof of (iv). We prove this by induction with respect to $\mathbf{A}$. Take $\phi(\mathbf{A})$ to be "whenever $\mathbf{B}<\mathbf{A}$ and $\mathbf{C}<\mathbf{A}$ then $\mathbf{B}<\mathbf{C}$ or $\mathbf{C}<\mathbf{B}$ or $\mathbf{B}$ conv $\mathbf{C}$ "." $\phi(U)$ follows from the fact that we never have $\mathbf{B}<U$. If we have $\phi(\mathbf{A})$ and $\mathbf{B}<\operatorname{Suc}(\mathbf{A})$, then either $\mathbf{B}<\mathbf{A}$ or $\mathbf{B} \operatorname{conv} \mathbf{A}$; for we can find $\mathbf{D}$ such that $\mathbf{B} \leqslant \mathbf{D}$, and then $\mathbf{D}<\operatorname{Suc}(\mathbf{A})$ can be proved without appealing either to (1) or (5); (4) does not apply, so we must have D conv A. Then, if $\mathbf{B}<\operatorname{Suc}(\mathbf{A})$ and $\mathbf{C}<\operatorname{Suc}(\mathbf{A})$, we have four possibilities,

$$
\begin{array}{ll}
\mathbf{B} \operatorname{conv} \mathbf{A}, & \mathbf{C} \operatorname{conv} \mathbf{A}, \\
\mathbf{B} \operatorname{conv} \mathbf{A}, & \mathbf{C}<\mathbf{A}, \\
\mathbf{B}<\mathbf{A}, & \mathbf{C} \operatorname{conv} \mathbf{A}, \\
\mathbf{B}<\mathbf{A}, & \mathbf{C}<\mathbf{A} .
\end{array}
$$

In the first case $\mathbf{B}$ conv $\mathbf{C}$, in the second $\mathbf{C}<\mathbf{B}$, in the third $\mathbf{B}<\mathbf{C}$, and in the fourth the induction hypothesis applies.

Now suppose that $\lambda u f x \cdot \mathbf{R}(\mathbf{n})$ is a C-K ordinal formula, that

$$
\lambda u f x . \mathbf{R}(\mathbf{n})<\lambda u f x . \mathbf{R}(S(\mathbf{n})) \text { and } \phi(\mathbf{R}(\mathbf{n})),
$$

for each positive integer $n$, and that $\mathbf{A}$ conv $\lambda u f x . u(\mathbf{R})$. Then, if $\mathbf{B}<\mathbf{A}$, this means that $\mathbf{B}<\lambda u f x \cdot \mathbf{R}(\mathbf{n})$ for some $n$; if we have also $\mathbf{C}<\mathbf{A}$, then $\mathbf{B}<\lambda u f x . \mathbf{R}(\mathbf{q}), \mathbf{C}<\lambda u f x . \mathbf{R}(\mathbf{q})$ for some $q$. Thus, for these $\mathbf{B}$ and $\mathbf{C}$, the required result follows from $\phi(\lambda u f x \cdot \mathbf{R}(\mathbf{q}))$.

Proof of $(\mathrm{v})$. The conditions $(C),(D)$ imply that the classes of interconvertible formulae $\mathbf{B}, \mathbf{B}<\mathbf{A}$ are well-ordered by the relation " $<$ ". We prove ( $v$ ) by (ordinary) transfinite induction with respect to the order type $a$ of the series formed by these classes; ( $a$ is, in fact, the solution of the 
equation $1+a=\Xi_{\mathrm{A}}$, but we do not need this). We suppose then that $(v)$ is true for all order types less than $\alpha$. If $\mathbf{E}<\mathbf{A}$, then $\mathbf{E}$ satisfies the conditions of $(v)$ and the corresponding order type is smaller: $\mathbf{E}$ is therefore a $\mathrm{C}-\mathrm{K}$ ordinal formula. This expresses all consequences of the induction hypothesis that we need. There are three cases to consider:

$$
\begin{aligned}
& \text { (x) } a=0 . \\
& \text { (y) } a=\beta+1 . \\
& (z) a \text { is of neither of the forms }(x),(y) .
\end{aligned}
$$

In case $(x)$ we must have $\mathbf{A}$ conv $U$ on account of $(\mathrm{A})$. In case $(y)$ there is a formula $\mathbf{D}$ such that $\mathbf{D}<\mathbf{A}$, and $\mathbf{B} \leqslant \mathbf{D}$ whenever $\mathbf{B}<\mathbf{A}$. The relation $\mathbf{D}<\mathbf{A}$ must hold in virtue of either (1), (2), (3), or (4). It cannot be in virtue of (4); for then there would be $\mathbf{B}, \mathbf{B}<\mathbf{A}, \mathbf{D}<\mathbf{B}$ contrary to (C), taken in conjunction with the definition of $D$. If it is in virtue of (3), then $a$ is the upper bound of a sequence $a_{1}, a_{2}, \ldots$ of ordinals, which are increasing by reason of (iii) and the conditions $\lambda u f x . \mathbf{R}(\mathbf{n})<\lambda u f x . \mathbf{R}(S(\mathbf{n}))$ in (B). This is inconsistent with $a=\beta+1$. This means that (2) applies [after we have eliminated (1) by suitable conversions on $\mathbf{A}, \mathbf{D}$ ] and we see that $\mathbf{A}$ conv $\operatorname{Suc}(\mathbf{D})$; but, since $\mathbf{D}<\mathbf{A}, \mathbf{D}$ is a $\mathbf{C}-\mathbf{K}$ ordinal formula, and $\mathbf{A}$ must therefore be a $\mathrm{C}-\mathrm{K}$ ordinal formula by (8). Now take case $(z)$. It is impossible for $\mathbf{A}$ to be of the form Suc (D), for then we should have $\mathbf{B}<\mathbf{D}$ whenever $\mathbf{B}<\mathbf{A}$, and this would mean that we had case $(y)$. Since $U<\mathbf{A}$, there must be an $\mathbf{F}$ such that $\mathbf{F}<\mathbf{A}$ is demonstrable either by (2) or by (3) (after a possible conversion on $\mathbf{A}$ ); it must of course be demonstrable by (3). Then $\mathbf{A}$ is of the form $\lambda u f x \cdot u(\mathbf{R})$. By (3), (B) we see that $\lambda u f x . \mathbf{R}(\mathbf{n})<\mathbf{A}$ for each positive integer $n$; each $\lambda u f x . \mathbf{R}(\mathbf{n})$ is therefore a C-K ordinal formula. Applying (9), (B) we see that A is a C-K ordinal formula.

Proof of (vi). To prove the first half, it is sufficient to find a method whereby from a $\mathrm{C}-\mathrm{K}$ ordinal formula $\mathrm{A}$ we can find the corresponding ordinal formula $\Omega$. For then there is a formula $H_{1}$ such that $H_{1}(\mathbf{a})$ conv $\mathbf{p}$ if $a$ is the G.R. of $\mathbf{A}$ and $p$ is that of $\Omega$. $H$ is then to be defined by

$$
H \rightarrow \lambda a \text {. form }\left(H_{1}(\operatorname{Gr}(a))\right) .
$$

The method of finding $\Omega$ may be replaced by a method of finding $\Omega(\mathbf{m}, \mathbf{n})$, given $\mathbf{A}$ and any two positive integers $m, n$. We shall arrange the method so that, whenever $\mathbf{A}$ is not an ordinal formula, either the calculation of the values does not terminate or else the values are not consistent with 
$\Omega$ being an ordinal formula. In this way we can prove the second half of (vi).

Let Ls be a formula such that $\operatorname{Ls}(\mathbf{A})$ enumerates the classes of formulae $\mathbf{B}, \mathbf{B}<\mathbf{A}[i . e$. if $\mathbf{B}<\mathbf{A}$ there is one and only one positive integer $n$ for which $\operatorname{Ls}(\mathbf{A}, \mathbf{n})$ conv B]. Then the rule for finding the value of $\Omega(\mathbf{m}, \mathbf{n})$ is as follows :-

First determine whether $U \leqslant \mathbf{A}$ and whether $\mathbf{A}$ is convertible to the form $\mathbf{r}($ Suc,$U)$. This terminates if $\mathbf{A}$ is a $\mathrm{C}-\mathrm{K}$ ordinal formula.

If $\mathbf{A}$ conv $\mathbf{r}(\mathrm{Suc}, U)$ and either $m>r+1$ or $n>r+1$, then the value is 4 . If $m<n \leqslant r+1$, the value is 2 . If $n<m \leqslant r+1$, the value is 1 . If $m=n \leqslant r+1$, the value is 3 .

If $\mathbf{A}$ is not convertible to this form, we determine whether either $\mathbf{A}$ or $\operatorname{Ls}(\mathbf{A}, \mathbf{m})$ is convertible to the form $\lambda u f x . u(\mathbf{R})$; and if either of them is, we verify that $\lambda u f x \cdot \mathbf{R}(\mathbf{n})<\lambda u f x \cdot \mathbf{R}(S(\mathbf{n}))$. We shall eventually come to an affirmative answer if $\mathbf{A}$ is a $\mathrm{C}-\mathrm{K}$ ordinal formula.

Having checked this, we determine concerning $m$ and $n$ whether $\operatorname{Ls}(\mathbf{A}, \mathbf{m})<\operatorname{Ls}(\mathbf{A}, \mathbf{n}), \operatorname{Ls}(\mathbf{A}, \mathbf{n})<\operatorname{Ls}(\mathbf{A}, \mathbf{m})$, or $m=n$, and the value is to be accordingly 1,2 , or 3 .

If $\mathbf{A}$ is a $\mathbf{C}-\mathbf{K}$ ordinal formula, this process certainly terminates. To see that the values so calculated correspond to an ordinal formula, and one representing $\Xi_{A}$, first observe that this is so when $\Xi_{A}$ is finite. In the other case (iii) and (iv) show that $\Xi_{B}$ determines a one-one correspondence between the ordinals $\beta, 1 \leqslant \beta \leqslant \Xi_{A}$, and the classes of interconvertible formulae $\mathbf{B}, \mathbf{B}<\mathbf{A}$. If we take $G(m, n)$ to be $\operatorname{Ls}(\mathbf{A}, \mathbf{m})<\operatorname{Ls}(\mathbf{A}, \mathbf{n})$, we see that $G(m, n)$ is the ordering relation of a series of order type $\dagger \Xi_{\mathbf{A}}$ and on the other hand that the values of $\Omega(\mathbf{m}, \mathbf{n})$ are related to $G(m, n)$ as on p. 179.

To prove the second half suppose that $A$ is not a $\mathrm{C}-\mathrm{K}$ ordinal formula. Then one of the conditions (A)-(D) in (v) must not be satisfied. If (A) is not satisfied we shall not obtain a result even in the calculation of $\boldsymbol{\Omega}(1,1)$. If (B) is not satisfied, we shall have for some positive integers $p$ and $q$,

$$
\operatorname{Ls}(\mathbf{A}, \mathbf{p}) \operatorname{conv} \lambda u f x . u(\mathbf{R})
$$

but not $\lambda u f x \cdot \mathbf{R}(\mathbf{q})<\lambda u f x \cdot \mathbf{R}(S(\mathbf{q}))$. Then the process of calculating $\boldsymbol{\Omega}(\mathbf{p}, \mathbf{q})$ will not terminate. In case of failure of $(\mathrm{C})$ or $(\mathrm{D})$ the values of $\boldsymbol{\Omega}(\mathbf{m}, \mathbf{n})$ may all be calculable, but if so conditions $(a)-(f)$, p. 179 , will be violated. Thus, if $\mathbf{A}$ is not a C-K ordinal formula, then $H(\mathbf{A})$ is not an ordinal formula.

† The order type is $\beta$, where $1+\beta=\Xi_{A}$; but $\beta=\Xi_{A}$, since $\Xi_{A}$ is infinite. 
I propose now to define three formulae Sum, Lim, Inf of importance in connection with ordinal formulae. Since they are comparatively simple, they will for once be given almost in full. The formula Ug is one with the property that $U g(\mathbf{m})$ is convertible to the formula representing the largest odd integer dividing $m$ : it is not given in full. $P$ is the predecessor function; $P(S(\mathbf{m}))$ conv $\mathbf{m}, P(\mathbf{l})$ conv $\mathbf{l}$.

$$
\begin{aligned}
& \mathrm{Al} \rightarrow \lambda p x y \cdot p(\lambda g u v \cdot g(v, u), \lambda u v \cdot u(I, v), x, y), \\
& \mathrm{Hf} \rightarrow \lambda m \cdot P(m(\lambda g u v \cdot g(v, S(u)), \lambda u v \cdot v(I, u), 1,2)), \\
& \mathrm{Bd} \rightarrow \lambda w w^{\prime} a a^{\prime} x \cdot \operatorname{Al}\left(\lambda f \cdot w\left(a, a, w^{\prime}\left(a^{\prime}, a^{\prime}, f\right)\right), x, 4\right),
\end{aligned}
$$$$
\operatorname{Sum} \rightarrow \lambda w w^{\prime} p q \cdot \operatorname{Bd}\left(w, w^{\prime}, \operatorname{Hf}(p), \operatorname{Hf}(q),\right.
$$

$$
\left.\operatorname{Al}\left(p, \operatorname{Al}\left(q, w^{\prime}(\operatorname{Hf}(p), \operatorname{Hf}(q)), 1\right), \operatorname{Al}(S(q), w(\operatorname{Hf}(p), \operatorname{Hf}(q)), 2)\right)\right),
$$

$\operatorname{Lim} \rightarrow \lambda z p q \cdot\{\lambda a b \cdot \mathrm{Bd}(z(a), z(b), \mathrm{Ug}(p), \mathrm{Ug}(q), \operatorname{Al}(\operatorname{Dt}(a, b)+\operatorname{Dt}(b, a)$,

$$
\operatorname{Dt}(a, b), z(a, \operatorname{Ug}(p), \operatorname{Ug}(q))))\}(\varpi(2, p), \varpi(2, q))
$$

$\operatorname{Inf} \rightarrow \lambda w a p q \cdot \operatorname{Al}(\lambda f \cdot w(a, p, w(a, q, f)), w(p, q), 4)$.

The essential properties of these formulae are described by:

$\operatorname{Al}(2 \mathbf{r}-1, \mathbf{m}, \mathbf{n})$ conv $\mathbf{m}, \quad \operatorname{Al}(2 \mathbf{r}, \mathbf{m}, \mathbf{n})$ conv $\mathbf{n}$,

$\mathrm{Hf}(2 \mathrm{~m})$ conv $\mathbf{m}, \quad \mathrm{Hf}(2 \mathrm{~m}-1)$ conv $\mathbf{m}$,

$\operatorname{Bd}\left(\Omega, \Omega^{\prime}, \mathbf{a}, \mathbf{a}^{\prime}, \mathbf{x}\right)$ conv 4 , unless both

$$
\boldsymbol{\Omega}(\mathbf{a}, \mathbf{a}) \operatorname{conv} 3 \text { and } \boldsymbol{\Omega}^{\prime}\left(\mathbf{a}^{\prime}, \mathbf{a}^{\prime}\right) \operatorname{conv} 3,
$$

it is then convertible to $\mathbf{x}$.

If $\Omega, \Omega^{\prime}$ are ordinal formulae representing $\alpha, \beta$ respectively, then $\operatorname{Sum}\left(\boldsymbol{\Omega}, \boldsymbol{\Omega}^{\prime}\right)$ is an ordinal formula representing $\alpha+\beta$. If $\mathbf{Z}$ is a W.F.F. enumerating a sequence of ordinal formulae representing $\alpha_{1}, a_{2}, \ldots$, then $\operatorname{Lim}(\mathbf{Z})$ is an ordinal formula representing the infinite sum $a_{1}+a_{2}+a_{3} \ldots$ 
If $\Omega$ is an ordinal formula representing $a$, then $\operatorname{Inf}(\boldsymbol{\Omega})$ enumerates a sequence of ordinal formulae representing all the ordinals less than a without repetitions other than repetitions of the ordinal 0 .

To prove that there is no general method for determining about a formula whether it is an ordinal formula, we use an argument akin to that leading to the Burali-Forti paradox; but the emphasis and the conclusion are different. Let us suppose that such an algorithm is available. This enables us to obtain a recursive enumeration $\Omega_{1}, \Omega_{2}, \ldots$ of the ordinal formulae in normal form. There is a formula $\mathbf{Z}$ such that $\mathbf{Z}(\mathbf{n})$ conv $\boldsymbol{\Omega}_{\mathbf{n}}$. Now $\operatorname{Lim}(\mathbf{Z})$ represents an ordinal greater than any represented by an $\Omega_{n}$, and it has therefore been omitted from the enumeration.

This argument proves more than was originally asserted. In fact, it proves that, if we take any class $E$ of ordinal formulae in normal form, such that, if $\mathbf{A}$ is any ordinal formula, then there is a formula in $E$ representing the same ordinal as $\mathbf{A}$, then there is no method whereby one can determine whether a W.F.F. in normal form belongs to $E$.

\section{s. Ordinal logics.}

An ordinal logic is a W.F.F. $\Lambda$ such that $\Lambda(\Omega)$ is a logic formula whenever $\Omega$ is an ordinal formula.

This definition is intended to bring under one heading a number of ways of constructing logics which have recently been proposed or which are suggested by recent advances. In this section I propose to show how to obtain some of these ordinal logics.

Suppose that we have a class $W$ of logical systems. The symbols used in each of these systems are the same, and a class of sequences of symbols called "formulae" is defined, independently of the particular system in $W$. The rules of procedure of a system $C$ define an axiomatic subset of the formulae, which are to be described as the "provable formulae of $C$ ". Suppose further that we have a method whereby, from any system $C$ of $W$, we can obtain a new system $C^{\prime}$, also in $W$, and such that the set of provable formulae of $C^{\prime}$ includes the provable formulae of $C$ (we shall be most interested in the case in which they are included as a proper subset). It is to be understood that this "method" is an effective procedure for obtaining the rules of procedure of $C^{\prime}$ from those of $C$.

Suppose that to certain of the formulae of $W$ we make numbertheoretic theorems correspond: by modifying the definition of formula, we may suppose that this is donef or all formulae. We shall say that one of the systems $C$ is valid if the provability of a formula in $C$ implies the truth of the corresponding number-theoretic theorem. Now let the relation of 
$C^{\prime}$ to $C$ be such that the validity of $C$ implies the validity of $C^{\prime}$, and let there be a valid system $C_{0}$ in $W$. Finally, suppose that, given any computable sequence $C_{1}, C_{2}, \ldots$ of systems in $W$, the "limit system", in which a formula is provable if and only if it is provable in one of the systems $C_{j}$, also belongs to $W$. These limit systems are to be regarded, not as functions of the sequence given in extension, but as functions of the rules of formation of their terms. A sequence given in extension may be described by various rules of formation, and there will be several corresponding limit systems. Each of these may be described as $a$ limit system of the sequence.

In these circumstances we may construct an ordinal logic. Let us associate positive integers with the systems in such a way that to each $C$ there corresponds a positive integer $m_{C}$, and that $m_{C}$ completely describes the rules of procedure of $C$. Then there is a W.F.F. K, such that

$$
\mathbf{K}\left(\mathbf{m}_{C}\right) \text { conv } \mathbf{m}_{C^{\prime}}
$$

for each $C$ in $W$, and there is a W.F.F. $\Theta$ such that, if $\mathbf{D}(\mathbf{r})$ conv $\mathbf{m}_{C_{r}}$ for each positive integer $r$, then $\Theta(\mathbf{D})$ conv $\mathbf{m}_{C}$, where $C$ is a limit system of $C_{1}, C_{2}, \ldots$ With each system $C$ of $W$ it is possible to associate a logic formula $\mathbf{L}_{C}$ : the relation between them is that, if $G$ is a formula of $W$ and the number-theoretic theorem corresponding to $G$ (assumed expressed in the conversion calculus form) asserts that $\mathbf{B}$ is dual, then $\mathbf{L}_{C}(\mathbf{B})$ conv 2 if and only if $G$ is provable in $C$. There is a W.F.F. G such that

$$
\mathbf{G}\left(\mathbf{m}_{C}\right) \operatorname{conv} \mathbf{L}_{C}
$$

for each $C$ of $W$. Put

$$
\mathbf{N} \rightarrow \lambda a \cdot \mathbf{G}\left(a\left(\Theta, \mathbf{K}, \mathbf{m}_{C_{0}}\right)\right) .
$$

I assert that $\mathbf{N}(\mathbf{A})$ is a logic formula for each $\mathrm{C}-\mathrm{K}$ ordinal formula $\mathbf{A}$, and that, if $\mathbf{A}<\mathbf{B}$, then $\mathbf{N}(B)$ is more complete than $\mathbf{N}(\mathbf{A})$, provided that there are formulae provable in $C^{\prime}$ but not in $C$ for each valid $C$ of $W$.

To prove this we shall show that to each $\mathrm{C}-\mathrm{K}$ ordinal formula $\mathbf{A}$ there corresponds a unique system $C[\mathbf{A}]$ such that:

(i) $\mathbf{A}\left(\Theta, \mathbf{K}, \mathbf{m}_{C_{0}}\right)$ conv $\mathbf{m}_{C[A]}$,

and that it further satisfies:

(ii) $C[U]$ is a limit system of $C_{0}{ }^{\prime}, C_{0}{ }^{\prime}, \ldots$,

(iii) $C[\operatorname{Suc}(\mathbf{A})]$ is $(C[\mathbf{A}])^{\prime}$,

(iv) $C[\lambda u f x . u(\mathbf{R})]$ is a limit system of $C[\lambda u f x . \mathbf{R}(1)], C[\lambda u f x . \mathbf{R}(2)], \ldots$, 
A and $\lambda u f x . u(\mathbf{R})$ being assumed to be C-K ordinal formulae. The uniqueness of the system follows from the fact that $m_{C}$ determines $C$ completely. Let us try to prove the existence of $C[\mathbf{A}]$ for each $\mathrm{C}-\mathrm{K}$ ordinal formula A. As we have seen (p. 182) it is sufficient to prove

(a) $C[U]$ exists,

(b) if $C[\mathbf{A}]$ exists, then $C[\operatorname{Suc}(\mathbf{A})]$ exists,

(c) if $C[\lambda u f x \cdot \mathbf{R}(1)], C[\lambda u f x \cdot \mathbf{R}(2)], \ldots$ exist, then $C[\lambda u f x . u(\mathbf{R})]$ exists.

Proof of $(a)$.

$$
\left\{\lambda y \cdot \mathbf{K}\left(y\left(I, \mathbf{m}_{C_{0}}\right)\right)\right\}(\mathbf{n}) \operatorname{conv} \mathbf{K}\left(\mathbf{m}_{C_{0}}\right) \operatorname{conv} \mathbf{m}_{C^{\prime}} .
$$

for all positive integers $n$, and therefore, by the definition of $\Theta$, there is a system, which wo call $C[U]$ and which is a limit system of $C_{0}{ }^{\prime}, C_{0}{ }^{\prime}, \ldots$, satisfying

$$
\boldsymbol{\Theta}\left(\lambda y \cdot \mathbf{K}\left(y\left(I, \mathbf{m}_{C_{0}}\right)\right)\right) \operatorname{conv} \mathbf{m}_{C[U]} .
$$

But, on the other hand,

$$
U\left(\Theta, \mathbf{K}, \mathbf{m}_{C_{0}}\right) \operatorname{conv} \Theta\left(\lambda y \cdot \mathbf{K}\left(y\left(I, \mathbf{m}_{C_{0}}\right)\right)\right) .
$$

This proves $(a)$ and incidentally (ii).

Proof of $(b)$.

$$
\begin{aligned}
\operatorname{Suc}\left(A, \Theta, \mathbf{K}, \mathbf{m}_{C_{0}}\right) & \operatorname{conv} \mathbf{K}\left(\mathbf{A}\left(\Theta, \mathbf{K}, \mathbf{m}_{C_{0}}\right)\right) \\
& \operatorname{conv} \mathbf{K}\left(\mathbf{m}_{C[A]}\right) \\
& \operatorname{conv} \mathbf{m}_{(C[\mathrm{~A}])^{\circ} .}
\end{aligned}
$$

Hence $C[\operatorname{Suc}(\mathbf{A})]$ exists and is given by (iii).

Proof of $(c)$.

$$
\begin{gathered}
\left\{\{\lambda u f x \cdot \mathbf{R}\}\left(\Theta, \mathbf{K}, \mathbf{m}_{C_{0}}\right)\right\}(\mathbf{n}) \operatorname{conv}\{\lambda u f x \cdot \mathbf{R}(\mathbf{n})\}\left(\Theta, \mathbf{K}, \mathbf{m}_{C_{0}}\right) \\
\operatorname{conv} \mathbf{m}_{C[\lambda u f x \cdot \mathbf{R}(\mathbf{n})]}
\end{gathered}
$$

by hypothesis. Consequently, by the definition of $\Theta$, there exists a $C$ which is a limit system of

$$
C[\lambda u f x \cdot \mathbf{R}(1)], \quad C[\lambda u f x \cdot \mathbf{R}(2)], \ldots,
$$


and satisfies

$$
\boldsymbol{\theta}\left(\{\lambda u f x . u(\mathbf{R})\}\left(\boldsymbol{\Theta}, \mathbf{K}, \mathbf{m}_{C_{0}}\right)\right) \operatorname{conv} \mathbf{m}_{C} .
$$

We define $C[\lambda u f x . u(\mathrm{R})]$ to be this $C$. We then have (iv) and

$$
\begin{gathered}
\{\lambda u f x . u(\mathbf{R})\}\left(\Theta, \mathbf{K}, \mathbf{m}_{C_{0}}\right) \operatorname{conv} \Theta\left(\{\lambda u f x . \mathbf{R}\}\left(\Theta, \mathbf{K}, \mathbf{m}_{C_{0}}\right)\right) \\
\operatorname{conv} \mathbf{m}_{C[\lambda u f x . u(\mathbf{R})]} .
\end{gathered}
$$

'This completes the proof of the properties (i)-(iv). From (ii), (iii), (iv), the fact that $C_{0}$ is valid, and that $C^{\prime}$ is valid when $C$ is valid, we infer that $C[\mathbf{A}]$ is valid for each $\mathrm{C}-\mathrm{K}$ ordinal formula $\mathbf{A}$ : also that there are more formulae provable in $C[\mathbf{B}]$ than in $C[\mathbf{A}]$ when $\mathbf{A}<\mathbf{B}$. The truth of our assertions regarding $\mathbf{N}$ now follows in view of (i) and the definitions of $\mathbf{N}$ and $\mathbf{G}$.

We cannot conclude that $\mathbf{N}$ is an ordinal logic, since the formulae $\mathbf{A}$ are C-K ordinal formulae; but the formula $H$ enables us to obtain an ordinal logic from $\mathbf{N}$. By the use of the formula Gr we obtain a formula 'Tn such that, if $\mathbf{A}$ has a normal form, then $\operatorname{Tn}(\mathbf{A})$ enumerates the G.R.'s of the formulae into which $\mathbf{A}$ is convertible. Also there is a formula $\mathrm{Ck}$ such that, if $h$ is the G.R. of a formula $H(\mathbf{B})$, then $\mathrm{Ck}(\mathbf{h})$ conv $\mathbf{B}$, but otherwise $\mathrm{Ck}(\mathbf{h})$ conv $U$. Since $H(\mathbf{B})$ is an ordinal formula only if $\mathbf{B}$ is a $\mathrm{C}-\mathrm{K}$ ordinal formula, $\mathrm{Ck}(\operatorname{Tn}(\boldsymbol{\Omega}, \mathbf{n}))$ is a $\mathrm{C}-\mathrm{K}$ ordinal formula for each ordinal formula $\Omega$ and each integer $n$. For many ordinal formulae it will be convertible to $U$, but, for suitable $\boldsymbol{\Omega}$, it will be convertible to any given C-K ordinal formula. If we put

$$
\Lambda \rightarrow \lambda w a . \Gamma(\lambda n \cdot \mathbf{N}(\mathrm{Ck}(\operatorname{Tn}(w, n))), a),
$$

$\Lambda$ is the required ordinal logic. In fact, on account of the properties of $\Gamma, \Lambda(\Omega, A)$ will be convertible to 2 if and only if there is a positive integer $n$ such that

$$
\mathbf{N}(\operatorname{Ck}(\operatorname{Tn}(\boldsymbol{\Omega}, \mathbf{n})), \mathbf{A}) \operatorname{conv} \mathbf{2}
$$

If $\Omega$ conv $H(\mathbf{B})$, there will be an integer $n$ such that $\operatorname{Ck}(\operatorname{Tn}(\Omega, \mathbf{n}))$ conv $\mathbf{B}$, and then

$$
\mathbf{N}(\operatorname{Ck}(\operatorname{Tn}(\Omega, \mathbf{n})), \mathbf{A}) \operatorname{conv} \mathbf{N}(\mathbf{B}, \mathbf{A})
$$


For any $n, \operatorname{Ck}(\operatorname{Tn}(\boldsymbol{\Omega}, \mathbf{n}))$ is convertible to $U$ or to some $\mathbf{B}$, where $\Omega$ conv $H(\mathbf{B})$. Thus $\Lambda(\Omega, \mathbf{A})$ conv 2 if $\Omega$ conv $H(\mathbf{B})$ and $\mathbf{N}(\mathbf{B}, \mathbf{A})$ conv 2 or if $\mathbf{N}(U, \mathbf{A})$ conv 2 , but not in any other case.

We may now specialize and consider particular classes $W$ of systems. First let us try to construct the ordinal logic described roughly in the introduction. For $W$ we take the class of systems arising from the system of Principia Mathematicat by adjoining to it axiomatic (in the sense described on p. 167) sets of axioms f. Gödel has shown that primitive recursive relations $\S$ can be expressed by means of formulae in $P$. In fact, there is a rule whereby, given the recursion equations defining a primitive recursive relation, we can find a formula|| $\mathfrak{A}\left[x_{0}, \ldots, z_{0}\right]$ such that

$$
\mathfrak{A}\left[f^{\left(m_{1}\right)} 0, \ldots, f^{\left(m_{r}\right)} 0\right]
$$

is provable in $P$ if $F\left(m_{1}, \ldots, m_{r}\right)$ is true, and its negation is provable otherwise. Further, there is a method by which we can determine about a formula $\mathfrak{A}\left[x_{0}, \ldots, z_{0}\right]$ whether it arises from a primitive recursive relation in this way, and by which we can find the equations which defined the relation. Formulae of this kind will be called recursion formulae. We shall make use of a property that they possess, which we cannot prove formally here without giving their definition in full, but which is essentially trivial. $\mathrm{Db}\left[x_{0}, y_{0}\right]$ is to stand for a certain recursion formula such that $\mathrm{Db}\left[f^{(m)} 0, f^{(n)} 0\right]$ is provable in $P$ if $m=2 n$ and its negation is provable otherwise. Suppose that $\mathfrak{A}\left[x_{0}\right], \mathfrak{B}\left[x_{0}\right]$ are two recursion formulae. Then the theorem which

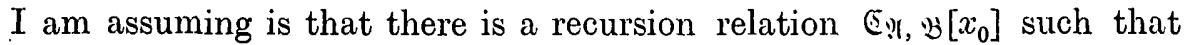
we can prove

$$
\mathfrak{S}_{\mathfrak{N}, \mathfrak{B}}\left[x_{0}\right] \equiv\left(\exists y_{0}\right)\left(\left(\mathrm{Db}\left[x_{0}, y_{0}\right] . \mathfrak{H}\left[y_{0}\right]\right) \vee\left(\mathrm{Db}\left[f x_{0}, f y_{0}\right] . \mathfrak{S}\left[y_{0}\right]\right)\right)
$$

in $P$.

$\dagger$ Whitehead and Russell [1]. The axioms and rules of procedure of a similar system $P$ will be found in a convenient form in Gödel [1], and I follow Gödel. The symbols for the natural numbers in $P$ are $0, f 0, f f 0, \ldots, f^{i n}, 0 \ldots$ Variables with the suffix " 0 " stand for natural numbers.

$\ddagger$ It is sometimes regarded as necessary that the set of axioms used should be computable, the intention being that it should be possible to verify of a formula reputed to be all axiom whether it really is so. We can obtain the same effect with axiomatic sets of axioms in this way. In the rules of procedure describing which are the axioms, we incorporate a method of enumerating them, and we also introduce a rule that in the main part of the deduction, whenever we write down an axiom as such, we must also write down its position in the enumeration. It is possible to verify whether this has been done correctly.

$\S$ A relation $F\left(m_{1}, \ldots, m_{r}\right)$ is primitive recursive if it is a necessary and sufficient condition for the vanishing of a primitive recursive function $\phi\left(m_{1}, \ldots, m_{r}\right)$.

|| Capital German letters will be used to stand for variable or undetermined formulae

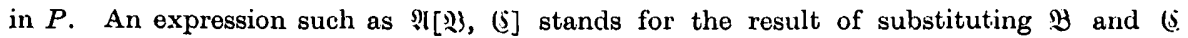
for $x_{0}$ and $y_{0}$ in $\mathfrak{R}$.

SKR. 2. vox. 45 . vo. 2241. 
The significant formulae in any of our extensions of $P$ are those of the form

$$
\left(x_{0}\right)\left(\exists y_{0}\right) \mathfrak{A}\left[x_{0}, y_{0}\right]
$$

where $\mathfrak{A}\left[x_{0}, y_{0}\right]$ is a recursion formula, arising from the relation $R(m, n)$ let us say. The corresponding number-theoretic theorem states that for each natural number $m$ there is a natural number $n$ such that $R(m, n)$ is true.

The systems in $W$ which are not valid are those in which a formula of the form (8.2) is provable, but at the same time there is a natural number, $m$ say, such that, for each natural number $n, R(m, n)$ is false. This means to say that $\sim \mathfrak{A}\left[f^{(m)} 0, f^{(n)} 0\right]$ is provable for each natural number $n$. Since (8.2) is provable, $\left(\exists x_{0}\right) \mathfrak{A}\left[f^{(m)} 0, y_{0}\right]$ is provable, so that

$$
\left(\exists y_{0}\right) \mathfrak{A}\left[f^{(m)} 0, y_{0}\right], \quad \sim \mathfrak{A}\left[f^{(m)} 0,0\right], \quad \sim \mathfrak{A}\left[f^{(m)} 0, f 0\right], \quad \ldots
$$

are all provable in the system. We may simplify (8.3). For a given $m$ we may prove a formula of the form $\mathfrak{A}\left[f^{(m)} 0, y_{0}\right] \equiv \mathfrak{B}\left[y_{0}\right]$ in $P$, where $\mathfrak{B}\left[x_{0}\right]$ is a recursion formula. Thus we find that a necessary and sufficient condition for a system of $W$ to be valid is that for no recursion formula $\mathfrak{B}\left[x_{0}\right]$ are all of the formulae

$$
\left(\exists x_{0}\right) \mathfrak{B}\left[x_{0}\right], \quad \sim \mathfrak{B}[0], \quad \sim \mathfrak{B}[f 0], \quad \ldots
$$

provable. An important consequence of this is that, if

$$
\mathfrak{A}_{1}\left[x_{0}\right], \quad \mathfrak{A}_{2}\left[x_{0}\right], \ldots, \mathfrak{A}_{n}\left[x_{0}\right]
$$

are recursion formulae, if

$$
\left(\exists x_{0}\right) \mathfrak{A}_{1}\left[x_{0}\right] \vee \ldots \vee\left(\exists x_{0}\right) \mathfrak{A}_{n}\left[x_{0}\right]
$$

is provable in $C$, and $C$ is valid, then we can prove $\mathfrak{A}_{r}\left[f^{(a)} 0\right]$ in $C$ for some natural numbers $r, a$, where $\mathrm{l} \leqslant r \leqslant n$. Let us define $\mathfrak{D}_{r}$ to be the formula

$$
\left(\exists x_{0}\right) \mathfrak{A}_{1}\left[x_{0}\right] \vee \ldots \vee\left(\exists x_{0}\right) \mathfrak{A}_{r}\left[x_{0}\right]
$$

and then define $\mathfrak{E}_{r}\left[x_{0}\right]$ recursively by the condition that $\mathfrak{E}_{1}\left[x_{0}\right]$ is $\mathfrak{A}_{1}\left[x_{0}\right]$ and $\mathfrak{F}_{r+1}\left[x_{0}\right]$ be $\mathfrak{E}_{\mathfrak{E}_{r}, \mathfrak{1} r+1}\left[x_{0}\right]$. Now I say that

$$
\mathfrak{D}_{r} \supset\left(\exists x_{0}\right) \mathfrak{E}_{r}\left[x_{0}\right]
$$


is provable for $1 \leqslant r \leqslant n$. It is clearly provable for $r=1$ : suppose it to be provable for a given $r$. We can prove

$$
\left(y_{0}\right)\left(\exists x_{0}\right) \mathrm{Db}\left[x_{0}, y_{0}\right]
$$

and

$$
\left(y_{0}\right)\left(\Xi x_{0}\right) \mathrm{Db}\left[f x_{0}, f y_{0}\right]
$$

from which we obtain

$$
\mathfrak{E}_{r}\left[y_{0}\right] \supset\left(\exists x_{0}\right)\left(\left(\mathrm{Db}\left[x_{0}, y_{0}\right] . \mathfrak{E}_{r}\left[y_{0}\right]\right) \vee\left(\mathrm{Db}\left[f x_{0}, f y_{0}\right] \cdot \mathfrak{A}_{r+1}\left[y_{0}\right]\right)\right)
$$

and

$$
\mathfrak{A}_{r+1}\left[y_{0}\right] \supset\left(\exists x_{0}\right)\left(\left(\mathrm{Db}\left[x_{0}, y_{0}\right] . \mathbb{E}_{r}\left[y_{0}\right]\right) \vee\left(\mathrm{Db}\left[f x_{0}, f y_{0}\right] \cdot \mathfrak{A}_{r+1}\left[y_{0}\right]\right)\right) .
$$

'These together with (8.1) yield

$$
\left(\exists y_{0}\right) \mathfrak{\Xi}_{r}\left[y_{0}\right] \vee\left(\exists y_{0}\right) \mathfrak{A}_{r+1}\left[y_{0}\right] \supset\left(\exists x_{0}\right) \mathfrak{E}_{\mathfrak{E}_{r}, \mathfrak{I}_{r+1}}\left[x_{0}\right],
$$

which is sufficient to prove (8.6) for $r+1$. Now, since (8.5) is provable in $C,\left(\exists x_{0}\right) \mathfrak{F}_{n}\left[x_{0}\right]$ must also be provable, and, since $C$ is valid, this means that $\mathfrak{F}_{n}\left[f^{(m)} 0\right]$ must be provable for some natural number $m$. From (8.1) and the definition of $\mathbb{E}_{n}\left[x_{0}\right]$ we see that this implies that $\mathfrak{A}_{r}\left[f^{(a)} 0\right]$ is provable for some natural numbers $a$ and $r, 1 \leqslant r \leqslant n$.

To any system $C$ of $W$ we can assign a primitive recursive relation $P_{C}(m, n)$ with the intuitive meaning " $m$ is the G.R. of a proof of the formula whose G.R. is $n$ ". We call the corresponding recursion formula $\operatorname{Proof}_{C}\left[x_{0}, y_{0}\right]\left(i . e . \operatorname{Proof}_{C}\left[f^{(m)} 0, f^{(n)} 0\right]\right.$ is provable when $P_{C}(m, n)$ is true, and its negation is provable otherwise). We can now explain what is the relation of a system $C^{\prime}$ to its predecessor $C$. The set of axioms which we adjoin to $P$ to obtain $C^{\prime}$ consists of those adjoined in obtaining $C$, together with all formulae of the form

$$
\left(\exists x_{0}\right) \operatorname{Proof}_{C}\left[x_{0}, f^{(m)} 0\right] \supset \mathfrak{F},
$$

where $m$ is the G.R. of $\mathfrak{F}$.

We want to show that a contradiction can be obtained by assuming $C^{\prime \prime}$ to be invalid but $C$ to be valid. Let us suppose that a set of formulae of the form (8.4) is provable in $C^{\prime}$. Let $\mathfrak{A}_{1}, \mathfrak{A}_{2}, \ldots, \mathfrak{A}_{k}$ be those axioms of $C^{\prime}$ of the form (8.7) which are used in the proof of $\left(\exists x_{0}\right) \mathfrak{B}\left[x_{0}\right]$. We may suppose that none of them is provable in $C$. Then by the deduction theorem we see that

$$
\left(\mathfrak{A}_{1}, \mathfrak{H}_{2} \ldots \mathfrak{A}_{k}\right) \supset\left(\exists x_{0}\right) \mathfrak{B}\left[x_{0}\right]
$$


is provable in $C$. Let $\mathfrak{A}_{l}$ be $\left(\exists x_{0}\right) \operatorname{Proof}_{C}\left[x_{0}, f^{\left(m_{l}\right)} 0\right] \supset \mathfrak{F}_{l}$. Then from (8.8) we find that

$$
\left(\exists x_{0}\right) \operatorname{Proof}_{C}\left[x_{0}, f^{\left(m_{1}\right)} 0\right] \vee \ldots v\left(\Xi x_{0}\right) \operatorname{Proof}_{C}\left[x_{0}, f^{\left(m_{k}\right)} 0\right] \vee\left(\exists x_{0}\right) \mathfrak{B}\left[x_{0}\right]
$$

is provable in $C$. It follows from a result which we have just proved that either $\mathfrak{B}\left[f^{(c)} 0\right]$ is provable for some natural number $c$, or else $\operatorname{Proof}_{C}\left[f^{(n)} 0, f^{\left(m_{l}\right)} 0\right]$ is provable in $C$ for some natural number $u$ and some $l, 1 \leqslant l \leqslant k$ : but this would mean that $\tilde{F}_{l}$ is provable in $C$ (this is one of the points where we assume the validity of $C$ ) and therefore also in $C^{\prime}$, contrary to hypothesis. Thus $\mathfrak{B}\left[f^{(c)} 0\right]$ must be provable in $C^{\prime}$; but we are also assuming $\sim \mathfrak{B}^{2}\left[f^{(c)} 0\right]$ to be provable in $C^{\prime}$. There is therefore a contradiction in $C^{\prime}$. Let us suppose that the axioms $\mathfrak{A}_{1}{ }^{\prime}, \ldots, \mathfrak{A}_{k^{\prime}}{ }^{\prime}$, of the form (8.7), when adjoined to $C$ are sufficient to obtain the contradiction and that none of these axioms is that provable in $C$. Then

$$
\sim \mathfrak{H}_{1}^{\prime} \mathrm{v} \sim \mathfrak{A}_{2}{ }^{\prime} \mathrm{v} \ldots \mathrm{v} \sim \mathfrak{H}_{k^{\prime}}{ }^{\prime}
$$

is provable in $C$, and if $)_{l}^{\prime}$ is $\left(\exists x_{0}\right) \operatorname{Proof}_{C}\left[x_{0}, f^{\left(m_{l}^{\prime}\right)} 0\right] \supset \mathscr{F}_{l}^{\prime}$ then

$$
\left.\left(\exists x_{0}\right] \operatorname{Proof}_{C}\left[x_{0}, f^{\left.\left(m_{1}\right)^{\prime}\right)} 0\right] \vee \ldots v\left(\exists x_{0}\right) \text { Proof }\left[x_{0}, f^{\left(m_{k^{\prime}}\right)}\right) 0\right]
$$

is provable in $C$. But, by repetition of a previous argument, this means that $\mathfrak{A}_{l}^{\prime}$ is provable for some $l, 1 \leqslant l \leqslant k^{\prime}$, contrary to hypothesis. This is the required contradiction.

We may now construct an ordinal logic in the manner described on pp. 190-193. We shall, however, carry out the construction in rather more detail, and with some modifications appropriate to the particular case. Each system $C$ of our set $W$ may be described by means of a W.F.F. $M_{C}$ which enumerates the G.R.'s of the axioms of $C$. There is a W.F.F. $E$ such that, if $a$ is the G.R. of some proposition $\mathscr{F}$, then $E\left(M_{C}, \mathbf{a}\right)$ is convertible to the G.R. of

$$
\left(\exists x_{0}\right) \operatorname{Proof}_{C}\left[x_{0}, f^{(a)} 0\right] \supset \mathfrak{F} .
$$

If a is not the G.R. of any proposition in $P$, then $E\left(M_{C}, \mathbf{a}\right)$ is to be convertible to the G.R. of $0=0$. From $E$ we obtain a W.F.F. $K$ such that $K\left(M_{C^{\prime}}, 2 \mathbf{n}+1\right)$ conv $M_{C}(\mathbf{n}), K\left(M_{C}, 2 \mathbf{n}\right)$ conv $E\left(M_{C}, \mathbf{n}\right)$. The successor system $C^{\prime}$ is defined by $K\left(M_{C}\right)$ conv $M_{C^{\prime}}$. Let us choose a formula $G$ such that $G\left(M_{C}, \mathbf{A}\right)$ conv 2 if and only if the number-theoretic theorem equivalent to "A is dual" is provable in $C$. Then we define $\Lambda_{P}$ by

$\Lambda_{P} \rightarrow \lambda w a \cdot \Gamma\left(\lambda y \cdot G\left(\mathrm{Ck}\left(\operatorname{Tn}(w, y), \lambda m n \cdot m(\varpi(2, n), \varpi(3, n)), K, M_{P}\right)\right), a\right)$.

This is an ordinal logic provided that $P$ is valid. 
Another ordinal logic of this type has in effect been introduced by Church $\dagger$. Superficially this ordinal logic seems to have no more in common with $\Lambda_{P}$ than that they both arise by the method which we have described, which uses C-K ordinal formulae. The initial systems are entirely different. However, in the relation between $C$ and $C^{\prime}$ there is an interesting analogy. In Church's method the step from $C$ to $C^{\prime}$ is performed by means of subsidiary axioms of which the most important (Church [2], p. $88,1_{m}$ ) is almost a direct translation into his symbolism of the rule that we may take any formula of the form (8.4) as an axiom. There are other extra axioms, however, in Church's system, and it is therefore not unlikely that it is in some respects more complete than $\Lambda_{P}$.

There are other types of ordinal logic, apparently quite unrelated to the type that we have so far considered. I have in mind two types of ordinal logic, both of which can be best described directly in terms of ordinal formulae without any reference to C-K ordinal formulae. I shall describe here a specimen $\Lambda_{H}$ of one of these types of ordinal logic. Ordinal logics of this kind were first considered by Hilbert (Hilbert [1], $183 \mathrm{ff}$ ), and have also been used by Tarski (Tarski [1], 395ff); see also Gödel [1], foot-note $48^{a}$.

Suppose that we have selected a particular ordinal formula $\Omega$. We shall construct a modification $P_{\Omega}$ of the system $P$ of Gödel (see foot-note $\dagger$ on p. 193. We shall say that a natural number $n$ is a type if it is either even or $2 p-1$, where $\Omega(\mathbf{p}, \mathbf{p})$ conv 3 . The definition of a variable in $P$ is to be modified by the condition that the only admissible subscripts are to be the types in our sense. Elementary expressions are then defined as in $P$ : in particular the definition of an elementary expression of type 0 is unchanged. An elementary formula is defined to be a sequence of symbols of the form $\mathfrak{A}_{m} \mathfrak{A}_{n}$, where $\mathfrak{A}_{m}, \mathfrak{A}_{n}$ are elementary expressions of types $m$, $n$ satisfying one of the conditions $(a),(b),(c)$.

(a) $m$ and $n$ are both even and $m$ exceeds $n$,

(b) $m$ is odd and $n$ is even,

(c) $m=2 p-1, n=2 q-1$, and $\Omega(\mathbf{p}, \mathbf{q})$ conv 2 .

With these modifications the formal development of $P_{\Omega}$ is the same as that of $P$. We want, however, to have a method of associating numbertheoretic theorems with certain of the formulae of $P_{\Omega}$. We cannot take over directly the association which we used in $P$. Suppose that $G$ is a

$\dagger$ In outline Church [1], 279-280. In greater detail Church [2], Chap. X. 
formula in $P$ interpretable as a number-theoretic theorem in the way described in the course of constructing $\Lambda_{P}$ (p. 193). Then, if every type suffix in $G$ is doubled, we shall obtain a formula in $P_{\Omega}$ which is to be interpreted as the same number-theoretic theorem. By the method of $\S 6$ we can now obtain from $P_{\Omega}$ a formula $L_{\Omega}$ which is a logic formula if $P_{\Omega}$ is valid; in fact, given $\Omega$ there is a method of obtaining $L \Omega$, so that there is a formula $\Lambda_{H}$ such that $\Lambda_{H}(\Omega)$ conv $L_{\Omega}$ for each ordinal formula $\Omega$.

Having now familiarized ourselves with ordinal logics by means of these examples we may begin to consider general questions concerning them.

\section{Completeness questions.}

The purpose of introducing ordinal logics was to avoid as far as possible the effects of Gödel's theorem. It is a consequence of this theorem, suitably modified, that it is impossible to obtain a complete logic formula, or (roughly speaking now) a complete system of logic. We were able, however, from a given system to obtain a more complete one by the adjunction as axioms of formulae, seen intuitively to be correct, but which the Gödel theorem shows are unprovable $\dagger$ in the original system; from this we obtained a yet more complete system by a repetition of the process, and so on. We found that the repetition of the process gave us a new system for each C-K ordinal formula. We should like to know whether this process suffices, or whether the system should be extended in other ways as well. If it were possible to determine about a W.F.F. in normal form whether it was an ordinal formula, we should know for certain that it was necessary to make extensions in other ways. In fact for any ordinal formula $\Lambda$ it would then be possible to find a single logic formula $L$ such that, if $\Lambda(\Omega, A)$ conv 2 for some ordinal formula $\Omega$, then $\mathbf{L}(\mathbf{A})$ conv 2. Since $\mathbf{L}$ must be incomplete, there must be formulae $\mathbf{A}$ for which $\Lambda(\Omega, A)$ is not convertible to 2 for any ordinal formula $\Omega$. However, in view of the fact, proved in $\S 7$, that there is no method of determining about a formula in normal form whether it is an ordinal formula, the case does not arise, and there is still a possibility that some ordinal logics may be complete in some sense. There is a quite natural way of defining completeness.

Definition of completeness of an ordinal logic. We say that an ordinal logic $\Lambda$ is complete if corresponding to each dual formula $\boldsymbol{A}$ there is an ordinal formula $\Omega_{\mathrm{A}}$ such that $\Lambda\left(\Omega_{\mathrm{A}}, \mathrm{A}\right)$ conv 2 .

$\dagger$ In the case of $P$ we adjoined all of the axioms $\left(\exists x_{0}\right)$ Proof $\left[x_{0}, f^{(m)} 0\right] \supset \mathbb{F}$, where $m$ is the G.R. of $\mathfrak{F} ;$ the Gödel thenrem shows that some of them are unprovable in $P$. 
As has been explained in $\S 2$, the reference in the definition to the existence of $\Omega_{A}$ for each $A$ is to be understood in the same naive way as any reference to existence in mathematics.

There is room for modification in this definition: we might require that there is a formula $\mathbf{X}$ such that $\mathbf{X}(\mathbf{A}) \operatorname{conv} \Omega_{\mathrm{A}}, \mathbf{X}(\mathbf{A})$ being an ordinal formula whenever $\mathbf{A}$ is dual. There is no need, however, to discuss the relative merits of these two definitions, because in all cases in which we prove an ordinal logic to be complete we shall prove it to be complete even in the modified sense; but in cases in which we prove an ordinal logic to be incomplete, we use the definition as it stands.

In the terminology of $\S 6, \Lambda$ is complete if the class of logics $\Lambda(\Omega)$ is complete when $\Omega$ runs through all ordinal formulae.

There is another completeness property which is related to this one. Let us for the moment describe an ordinal logic $\Lambda$ as all inclusive if to each logic formula $L$ there corresponds an ordinal formula $\Omega_{(\mathrm{L})}$ such that $\Lambda\left(\Omega_{(\mathrm{L})}\right)$ is as complete as $L$. Clearly every all.inclusive ordinal logic is complete; for, if $\mathbf{A}$ is dual, then $\delta(\mathbf{A})$ is a logic with $\mathbf{A}$ in its extent. But, if $\Lambda$ is complete and

$$
\mathrm{Ai} \rightarrow \lambda k w . \Gamma(\lambda r a . \delta(4, \delta(2, k(w, V(\mathrm{Nm}(r))))+\delta(2, \mathrm{Nm}(r, a)))),
$$

then $\operatorname{Ai}(\Lambda)$ is an all inclusive ordinal logic. For, if $\mathbf{A}$ is in the extent of $\Lambda\left(\Omega_{\mathrm{A}}\right)$ for each $A$, and we put $\Omega_{(\mathrm{L})} \rightarrow \Omega_{V_{(\mathrm{L})}}$, then I say that, if $\mathbf{B}$ is in the extent of $L$, it must be in the extent of $\operatorname{Ai}\left(\Lambda, \Omega_{(L)}\right)$. In fact, we see that $\operatorname{Ai}\left(\Lambda, \Omega_{V(\mathbf{L})}, \mathbf{B}\right)$ is convertible to

$$
\Gamma\left(\lambda r a . \delta\left(4, \delta\left(2, \Lambda\left(\Omega_{\nabla(\mathbf{L})}, V(\mathrm{Nm}(r))\right)\right)+\delta(2, \mathrm{Nm}(r, a))\right), \mathbf{B}\right) .
$$

For suitable $n, \mathbf{N m}(\mathbf{n})$ conv $\mathbf{L}$ and then

$$
\Lambda\left(\Omega_{\nabla(\mathbf{L})}, V(\mathrm{Nm}(\mathbf{n}))\right) \operatorname{conv} 2,
$$

$$
\mathrm{Nm}(\mathbf{n}, \mathbf{B}) \text { conv } 2,
$$

and therefore, by the properties of $\Gamma$ and $\delta$

$$
\operatorname{Ai}\left(\Lambda, \Omega_{V(L)}, \text { B) conv } 2 .\right.
$$

Conversely $\operatorname{Ai}\left(\Lambda, \Omega_{V(\mathrm{~L})}\right.$, B) can be convertible to 2 only if both $\mathrm{Nm}(\mathbf{n}, \mathbf{B})$ 
and $\Lambda\left(\Omega_{V^{\prime}(\mathbf{L})}, V(\mathrm{Nm}(\mathbf{n}))\right)$ are convertible to 2 for some positive integer $n$; but, if $\Lambda\left(\Omega_{V^{\prime}(\mathrm{L})}, V(\mathrm{Nm}(\mathbf{n}))\right)$ conv 2 , then $\mathrm{Nm}(\mathbf{n})$ must be a logic, and, since $\mathrm{Nm}(\mathbf{n}, \mathbf{B})$ conv 2, B must be dual.

It should be noticed that our definitions of completeness refer only to number-theoretic theorems. Although it would be possible to introduce formulae analogous to ordinal logics which would prove more general theorems than number-theoretic ones, and have a corresponding definition of completeness, yet, if our theorems are too general, we shall find that our (modified) ordinal logics are never complete. This follows from the argument of $\S 4$. If our "oracle" tells us, not whether any given numbertheoretic statement is true, but whether a given formula is an ordinal formula, the argument still applies, and we find that there are classes of problem which cannot be solved by a uniform process even with the help of this oracle. This is equivalent to saying that there is no ordinal logic of the proposed modified type which is complete with respect to these problems. This situation becomes more definite if we take formulae satisfying conditions $(a)-(e),\left(f^{\prime}\right)$ (as described at the end of $\left.\S 12\right)$ instead of ordinal formulae; it is then not possible for the ordinal logic to be complete with respect to any class of problems more extensive than the number-theoretic problems.

We might hope to obtain some intellectually satisfying system of logical inference (for the proof of number-theoretic theorems) with some ordinal logic. Gödel's theorem shows that such a system cannot be wholly mechanical; but with a complete ordinal logic we should be able to confine the non-mechanical steps entirely to verifications that particular formulae are ordinal formulae.

We might also expect to obtain an interesting classification of numbertheoretic theorems according to "depth". A theorem which required an ordinal $\alpha$ to prove it would be deeper than one which could be proved by the use of an ordinal $\beta$ less than $a$. However, this presupposes more than is justified. We now define

Invariance of ordinal logics. An ordinal logic $\Lambda$ is said to be invariant up to an ordinal $a$ if, whenever $\Omega, \Omega^{\prime}$ are ordinal formulae representing the same ordinal less than $\alpha$, the extent of $\Lambda(\Omega)$ is identical with the extent of $\Lambda\left(\Omega^{\prime}\right)$. An ordinal logic is invariant if it is invariant up to each ordinal represented by an ordinal formula.

Clearly the classification into depths presupposes that the ordinal logic used is invariant, 
Among the questions that we should now like to ask are

(a) Are there any complete ordinal logics?

(b) Are there any complete invariant ordinal logics?

To these we might have added "are all ordinal logics complete?"; but this is trivial; in fact, there are ordinal logics which do not suffice to prove any number-theoretic theorems whatever.

We shall now show that $(a)$ must be answered affirmatively. In fact, we can write down a complete ordinal logic at once. Put

$$
\operatorname{Od} \rightarrow \lambda a \cdot\{\lambda f m n . \operatorname{Dt}(f(m), f(n))\}(\lambda s . \varphi(\lambda r . r(I, a(s)), 1, s)))
$$

and

$$
\operatorname{Comp} \rightarrow \lambda w a . \delta(w, \operatorname{Od}(a)) .
$$

I shall show that Comp is a complete ordinal logic.

For if, $\operatorname{Comp}(\Omega, \mathbf{A})$ conv 2 , then

$\Omega \operatorname{conv} \operatorname{Od}(\mathbf{A})$

$$
\operatorname{conv} \lambda m n . \operatorname{Dt}(\mathscr{P}(\lambda r \cdot r(I, \mathbf{A}(m)), 1, m), \rho(\lambda r . r(I, \mathbf{A}(n)), 1, n))) .
$$

$\Omega(\mathbf{m}, \mathbf{n})$ has a normal form if $\Omega$ is an ordinal formula, so that then

$$
\odot(\lambda r \cdot r(I, \mathbf{A}(\mathbf{m})), \mathbf{l})
$$

has a normal form; this means that $\mathbf{r}(I, \mathbf{A}(\mathbf{m}))$ conv 2 some $r$, i.e. $\mathbf{A}(\mathbf{m})$ conv 2. Thus, if $\operatorname{Comp}(\Omega, A)$ conv 2 and $\Omega$ is an ordinal formula, then $\mathbf{A}$ is dual. Comp is therefore an ordinal logic. Now suppose conversely that $\mathbf{A}$ is dual. I shall show that $\operatorname{Od}(\mathbf{A})$ is an ordinal formula representing the ordinal $\omega$. For

$$
\begin{aligned}
& \mathscr{P}(\lambda r . r(I, \mathbf{A}(\mathbf{m})), \mathbf{l}, \mathbf{m}) \operatorname{conv} \mathscr{P}(\lambda r . r(I, 2), \mathbf{l}, \mathbf{m}) \\
& \operatorname{conv} \mathbf{l}(\mathbf{m}) \text { conv } \mathbf{m} \text {, } \\
& \operatorname{Od}(\mathbf{A}, \mathbf{m}, \mathbf{n}) \text { conv } \operatorname{Dt}(\mathbf{m}, \mathbf{n}) \text {, }
\end{aligned}
$$

i.e. $\operatorname{Od}(\mathbf{A})$ is an ordinal formula representing the same ordinal as Dt. But

$$
\operatorname{Comp}(\operatorname{Od}(\mathbf{A}), \mathbf{A}) \operatorname{conv} \delta(\operatorname{Od}(\mathbf{A}), \operatorname{Od}(\mathbf{A})) \operatorname{conv} 2 .
$$

This proves the completeness of Comp. 
Of course Comp is not the kind of complete ordinal logic that we should really wish to use. The use of Comp does not make it any easier to see that $\mathbf{A}$ is dual. In fact, if we really want to use an ordinal logic a proof, of completeness for that particular ordinal logic will be of little value; the ordinals given by the completeness proof will not be ones which can easily be seen intuitively to be ordinals. The only value in a completeness proof of this kind would be to show that, if any objection is to be raised against an ordinal logic, it must be on account of something more subtle than incompleteness.

The theorem of completeness is also unexpected in that the ordinal formulae used are all formulae representing $\omega$. This is contrary to our intentions in constructing $\Lambda_{P}$ for instance; implicitly we had in mind large ordinals expressed in a simple manner. Here we have small ordinals expressed in a very complex and artificial way.

Before trying to solve the problem (b), let us see how far $\Lambda_{P}$ and $\Lambda_{H}$ are invariant. We should certainly not expect $\Lambda_{P}$ to be invariant, since the extent of $\Lambda_{P}(\Omega)$ will depend on whether $\Omega$ is convertible to a formula of the form $H(\mathbf{A})$ : but suppose that we call an ordinal logic $\Lambda$ " $\mathrm{C}-\mathrm{K}$ invariant up to $a$ " if the extent of $\Lambda(H(\mathbf{A}))$ is the same as the extent of $\Lambda(H(\mathbf{B}))$ whenever $\mathbf{A}$ and $\mathbf{B}$ are $\mathbf{C}-\mathrm{K}$ ordinal formulae representing the same ordinal less than $a$. How far is $\Lambda_{P} \mathrm{C}-\mathrm{K}$ invariant? It is not difficult to see that it is C-K invariant up to any finite ordinal, that is to say up to $\omega$. It is also $\mathrm{C}-\mathrm{K}$ invariant up to $\omega+1$, as follows from the fact that the extent of

$$
\Lambda_{P}(H(\lambda u f x . u(\mathbf{R})))
$$

is the set-theoretic sum of the extents of

$$
\Lambda_{P}(H(\lambda u f x \cdot \mathbf{R}(1))), \quad \Lambda_{P}(H(\lambda u f x \cdot \mathbf{R}(2))), \quad \ldots
$$

However, there is no obvious reason for believing that it is $\mathrm{C}-\mathrm{K}$ invariant up to $\omega+2$, and in fact it is demonstrable that this is not the case (see the end of this section). Let us find out what happens if we try to prove that the extent of

$$
\Lambda_{P}\left(H\left(\operatorname{Suc}\left(\lambda u f x \cdot u\left(\mathbf{R}_{1}\right)\right)\right)\right)
$$

is the same as the extent of

$$
\Lambda_{P}\left(H\left(\operatorname{Suc}\left(\lambda u f x \cdot u\left(\mathbf{R}_{2}\right)\right)\right)\right)
$$


where $\lambda u f x . u\left(\mathbf{R}_{1}\right)$ and $\lambda u f x . u\left(\mathbf{R}_{2}\right)$ are two C-K ordinal formulae representing $\omega$. We should have to prove that a formula interpretable as a number-theoretic theorem is provable in $C\left[\operatorname{Suc}\left(\lambda u f x . u\left(\mathbf{R}_{1}\right)\right)\right]$ if, and only if, it is provable in $C\left[\operatorname{Suc}\left(\lambda u f x . u\left(\mathbf{R}_{2}\right)\right)\right]$. Now $C\left[\operatorname{Suc}\left(\lambda u f x . u\left(\mathbf{R}_{1}\right)\right)\right]$ is obtained from $C\left[\lambda u f x . u\left(\mathbf{R}_{1}\right)\right]$ by adjoining all axioms of the form

$$
\left(\exists x_{0}\right) \operatorname{Proof}_{C\left[\lambda u f x \cdot u\left(\mathbf{R}_{1}\right)\right]}\left[x_{0}, f^{(m)} 0\right) \supset \mathscr{F},
$$

where $m$ is the G.R. of $\mathscr{F}$, and $C\left[\operatorname{Suc}\left(\lambda u f x . u\left(\mathbf{R}_{2}\right)\right)\right]$ is obtained from $C\left[\lambda u f x . u\left(\mathbf{R}_{2}\right)\right]$ by adjoining all axioms of the form

$$
\left(\exists x_{0}\right) \operatorname{Proof}_{C\left[\lambda u f x . u\left(\mathbf{R}_{2}\right)\right]}\left[x_{0}, f^{(m)} 0\right] \supset \mathfrak{F} \text {. }
$$

The axioms which must be adjoined to $P$ to obtain $C\left[\lambda u f x . u\left(\mathbf{R}_{1}\right)\right]$ are essentially the same as those which must be adjoined to obtain the system $C\left[\lambda u f x . u\left(\mathbf{R}_{2}\right)\right]$ : however the rules of procedure which have to be applied before these axioms can be written down are in general quite different in the two cases. Consequently (9.1) and (9.2) are quite different axioms, and there is no reason to expect their consequences to be the same. A proper understanding of this will make our treatment of question (b) much more intelligible. See also footnote $\ddagger$ on page 193.

Now let us turn to $\Lambda_{H}$. This ordinal logic is invariant. Suppose that $\Omega$, $\Omega^{\prime}$ represent the same ordinal, and suppose that we have a proof of a numbertheoretic theorem $G$ in $P_{\Omega}$. The formula expressing the number-theoretic theorem does not involve any odd types. Now there is a one-one correspondence between the odd types such that if $2 m-1$ corresponds to $2 m^{\prime}-1$ and $2 n-1$ to $2 n^{\prime}-1$ then $\Omega(\mathbf{m}, \mathbf{n})$ conv 2 implies $\Omega^{\prime}\left(\mathbf{m}^{\prime}, \mathbf{n}^{\prime}\right)$ conv 2 . Let us modify the odd type-subscripts occurring in the proof of $G$, replacing each by its mate in the one-one correspondence. There results a proof in $P_{\Omega^{\prime}}$ with the same end formula $G$. That is to say that if $G$ is provable in $P_{\Omega}$ it is provable in $P_{\Omega^{\prime}} . \Lambda_{H}$ is invariant.

The question $(b)$ must be answered negatively. Much more can be proved, but we shall first prove an even weaker result which can be established very quickly, in order to illustrate the method.

I shall prove that an ordinal logic $\Lambda$ cannot be invariant and have the property that the extent of $\Lambda(\Omega)$ is a strictly increasing function of the ordinal represented by $\Omega$. Suppose that $\Lambda$ has these properties; then we shall obtain a contradiction. Let $\mathrm{A}$ be a W.F.F. in normal form and without free variables, and consider the process of carrying out conversions on $\mathbf{A}(1)$ until we have shown it convertible to 2 , then converting $\mathbf{A}(2)$ to 2 , then $\mathbf{A}$ (3) and so on: suppose that after $r$ steps we are still performing the 
conversion on $\mathbf{A}\left(\mathbf{m}_{r}\right)$. There is a formula $\mathrm{Jh}$ such that $\mathrm{Jh}(\mathbf{A}, \mathbf{r})$ conv $\mathbf{m}_{r}$ for each positive integer $r$. Now let $Z$ be a formula such that, for each positive integer $n, Z(\mathbf{n})$ is an ordinal formula representing $\omega^{n}$, and suppose B to be a member of the extent of $\Lambda(\operatorname{Suc}(\operatorname{Lim}(Z)))$ but not of the extent of $\Lambda(\operatorname{Lim}(Z))$. Put

$$
\mathbf{K}^{*} \rightarrow \lambda a \cdot \Lambda(\operatorname{Suc}(\operatorname{Lim}(\lambda r \cdot Z(\operatorname{Jh}(a, r)))), \mathbf{B}) ;
$$

then $\mathbf{K} *$ is a complete logic. For, if $\mathbf{A}$ is dual, then

$$
\operatorname{Suc}(\operatorname{Lim}(\lambda r . Z(\operatorname{Jh}(\mathbf{A}, r))))
$$

represents the ordinal $\omega^{\omega}+1$, and therefore $K^{*}(\mathbf{A})$ conv 2 ; but, if $\mathbf{A}(\mathbf{c})$ is not convertible to 2 , then

$$
\operatorname{Suc}(\operatorname{Lim}(\lambda r \cdot Z(\operatorname{Jh}(\mathbf{A}, r))))
$$

represents an ordinal not exceeding $\omega^{c}+1$, and $\mathbf{K}^{*}(\mathbf{A})$ is therefore not convertible to 2 . Since there are no complete logic formulae, this proves our assertion.

We may now prove more powerful results.

Incompleteness theorems. (A) If an ordinal logic $\Lambda$ is invariant up to an ordinal $\alpha$, then for any ordinal formula $\Omega$ representing an ordinal $\beta$, $\beta<\alpha$, the extent of $\Lambda(\Omega)$ is contained in the (set-theoretic) sum of the extents of the logics $\Lambda(\mathbf{P})$, where $\mathbf{P}$ is finite.

(B) If an ordinal logic $\Lambda$ is $\mathrm{C}-\mathrm{K}$ invariant up to an ordinal $a$, then for any $\mathrm{C}-\mathrm{K}$ ordinal formula $\mathrm{A}$ representing an ordinal $\beta, \beta<\alpha$, the extent of $\Lambda(H(\mathrm{~A}))$ is contained in the (set-theoretic) sum of the extents of the $\operatorname{logics} \Lambda(H(\mathbf{F}))$, where $\mathbf{F}$ is a $\mathrm{C}-\mathrm{K}$ ordinal formula representing an ordinal less than $\omega^{2}$.

Proof of (A). It is sufficient to prove that, if $\Omega$ represents an ordinal $\gamma$, $\omega \leqslant \gamma<\alpha$, then the extent of $\Lambda(\Omega)$ is contained in the set-theoretic sum of the extents of the logics $\Lambda\left(\Omega^{\prime}\right)$, where $\Omega^{\prime}$ represents an ordinal less than $\gamma$. The ordinal $\gamma$ must be of the form $\gamma_{0}+\rho$, where $\rho$ is finite and represented by $\mathbf{P}$ say, and $\gamma_{0}$ is not the successor of any ordinal and is not less than $\omega$. There are two cases to consider; $\gamma_{0}=\omega$ and $\gamma_{0} \geqslant 2 \omega$. In each of them we shall obtain a contradiction from the assumption that there is a W.F.F. 
B such that $\Lambda(\Omega$, B $)$ conv 2 whenever $\Omega$ represents $\gamma$, but is not convertible to 2 if $\Omega$ represents a smaller ordinal. Let us take first the case $\gamma_{0} \geqslant 2 \omega$. Suppose that $\gamma_{0}=\omega+\gamma_{1}$, and that $\Omega_{1}$ is an ordinal formula representing $\gamma_{1}$. Let $\mathbf{A}$ be any W.F.F. with a normal form and no free variables, and let $Z$ be the class of those positive integers which are exceeded by all integers $n$ for which $\mathbf{A}(\mathbf{n})$ is not convertible to 2 . Let $E$ be the class of integers $2 p$ such that $\Omega(\mathbf{p}, \mathbf{n})$ conv 2 for some $n$ belonging to $Z$. The class $E$, together with the class $Q$ of all odd integers, is constructively enumerable. It is evident that the class can be enumerated with repetitions, and since it is infinite the required enumeration can be obtained by striking out the repetitions. There is, therefore, a formula En such that $\operatorname{En}(\Omega, \mathbf{A}, \mathbf{r})$ runs through the formulae of the class $E+Q$ without repetitions as $r$ runs through the positive integers. We definc

$$
\mathrm{Rt} \rightarrow \lambda w a m n . \operatorname{Sum}(\mathrm{Dt}, w, \operatorname{En}(w, u, m), \operatorname{En}(w, u, u)) .
$$

'Then $\operatorname{Rt}\left(\Omega_{1}, A\right)$ is an ordinal formula which represents $\gamma_{0}$ if $A$ is dual, but a smaller ordinal otherwise. In fact

$$
\operatorname{Rt}\left(\Omega_{1}, \mathbf{A}, \mathbf{m}, \mathbf{n}\right) \operatorname{conv}\left\{\operatorname{Sum}\left(\operatorname{Dt}, \Omega_{1}\right)\right\}\left(\operatorname{En}\left(\Omega_{1}, \mathbf{A}, \mathbf{m}\right), \operatorname{En}\left(\Omega_{1}, \mathbf{A}, \mathbf{n}\right)\right) .
$$

Now, if $\mathbf{A}$ is dual, $E+Q$ includes all integers $m$ for which

$$
\left\{\operatorname{Sum}\left(\mathrm{Dt}, \Omega_{1}\right)\right\}(\mathbf{m}, \mathbf{m}) \text { conv } 3 .
$$

(This depends on the particular form that we have chosen for the formula Sum.) Putting " $\operatorname{En}\left(\Omega_{1}, \mathbf{A}, \mathbf{p}\right)$ conv q" for $M(p, q)$, we see that condition (7.4) is satisfied, so that $\operatorname{Rt}\left(\Omega_{1}, A\right)$ is an ordinal formula representing $\gamma_{0}$. But, if $\mathrm{A}$ is not dual, the set $E+Q$ consists of all integers $m$ for which

$$
\left\{\operatorname{Sum}\left(\mathrm{Dt}, \Omega_{1}\right)\right\}(\mathbf{m}, \mathbf{r}) \operatorname{conv} 2 \text {, }
$$

where $r$ depends only on $\mathbf{A}$. In this case $\operatorname{Rt}\left(\Omega_{1}, \mathbf{A}\right)$ is an ordinal formula representing the same ordinal as $\operatorname{Inf}\left(\operatorname{Sum}\left(\operatorname{Dt}, \Omega_{1}\right), \mathbf{r}\right)$, and this is smaller than $\gamma_{0}$. Now consider $\mathbf{K}$ :

$$
\mathbf{K} \rightarrow \lambda a \cdot \Lambda\left(\operatorname{Sum}\left(\operatorname{Rt}\left(\Omega_{1}, \mathbf{A}\right), \mathbf{P}\right), \mathbf{B}\right) .
$$

If $\mathbf{A}$ is dual, $\mathbf{K}(\mathbf{A})$ is convertible to 2 since $\operatorname{Sum}\left(\operatorname{Rt}\left(\Omega_{1}, \mathbf{A}\right), \mathbf{P}\right)$ represents $\gamma$. But, if $A$ is not dual, it is not convertible to 2 , since $\operatorname{Sum}\left(\operatorname{Rt}\left(\Omega_{1}, A\right), \mathbf{P}\right)$ then represents an ordinal smaller than $\gamma$. In $\mathbf{K}$ we therefore have a complete logic formula, which is impossible.

Now we take the case $\gamma_{0}=\omega$. We introduce a W.F.F. Mg such that if $n$ is the D.N. of a computing machine $\mathbb{N}_{\text {, and }}$, if by the $m$-th complete 
configuration of.$U$ the figure 0 has been printed, then $\mathrm{Mg}(\mathbf{n}, \mathbf{m})$ is convertible to $\lambda p q \cdot \operatorname{Al}(4(P, 2 p+2 q), 3,4)$ (which is an ordinal formula representing the ordinal 1 ), but if 0 has not been printed it is convertible to $\lambda p q \cdot p(q, I, 4)$ (which represents 0 ). Now consider

$$
\mathbf{M} \rightarrow \lambda n . \Lambda(\operatorname{Sum}(\operatorname{Lim}(\operatorname{Mg}(n)), \mathbf{P}), \mathbf{B}) .
$$

If the machine never prints 0 , then $\operatorname{Lim}(\lambda r . \operatorname{Mg}(\mathbf{n}, r))$ represents $\omega$ and $\operatorname{Sum}(\operatorname{Lim}(\operatorname{Mg}(\mathbf{n})), \mathbf{P})$ represents $\gamma$. This means that $\mathbf{M}(\mathbf{n})$ is convertible to 2. If, however, 11 never prints $0, \operatorname{Sum}(\operatorname{Lim}(\operatorname{Mg}(\mathbf{n})), \mathbf{P})$ represents a finite ordinal and $\mathbf{M}(\mathbf{n})$ is not convertible to 2 . In $\mathbf{M}$ we therefore have means of determining about a machine whether it ever prints 0 , which is impossible $\nmid$ (Turing [1], §8). This completes the proof of (A).

Proof of (B). It is sufficient to prove that, if $\mathrm{C}$ represents an ordinal $\gamma$, $\omega^{2} \leqslant \gamma<\alpha$, then the extent of $\Lambda(H(C))$ is included in the set-theoretic sum of the extents of $\Lambda(H(\mathbf{G}))$, where $\mathbf{G}$ represents an ordinal less than $\gamma$. We obtain a contradiction from the assumption that there is a formula $\mathbf{B}$ which is in the extent of $\Lambda(H(\mathbf{G}))$ if $\mathbf{G}$ represents $\gamma$, but not if it represents any smaller ordinal. The ordinal $\gamma$ is of the form $\delta+\omega^{2}+\xi$, where $\xi<\omega^{2}$. Let $\mathbf{D}$ be a $\mathrm{C}-\mathrm{K}$ ordinal formula representing $\delta$ and $\lambda u f x . \mathbf{Q}(u, f, \mathbf{A}(u, f, x))$ one representing $a+\xi$ whenever $\mathbf{A}$ represents $a$.

We now define a formula $\mathrm{Hg}$. Suppose that $\mathbf{A}$ is a W.F.F. in normal form and without free variables; consider the process of carrying out conversions on $\mathbf{A}(1)$ until it is brought into the form 2 , then converting $\mathbf{A}(2)$ to 2 , then $\mathrm{A}(3)$, and so on. Suppose that at the $r$-th step of this process we are doing the $n_{r}$-th step in the conversion of $\mathbf{A}\left(\mathbf{m}_{\mathbf{r}}\right)$. Thus, for instance, if A is not convertible to $2, m_{r}$ can never exceed 3. Then $\mathrm{Hg}(\mathbf{A}, \mathbf{r})$ is to be convertible to $\lambda f \cdot f\left(\mathbf{m}_{\mathbf{r}}, \mathbf{n}_{\mathbf{r}}\right)$ for each positive integer $r$. Put

$$
\begin{aligned}
& \mathrm{Sq} \rightarrow \lambda d m n \cdot n(\text { Suc, } m(\lambda a u f x \cdot u(\lambda y \cdot y(\operatorname{Suc}, a(u, f, x))), d(u, f, x))), \\
& \mathbf{M} \rightarrow \lambda a u f x \cdot \mathbf{Q}(u, f, u(\lambda y \cdot \operatorname{Hg}(a, y, \operatorname{Sq}(\mathbf{D})))), \\
& \mathbf{K}_{\mathbf{1}} \rightarrow \lambda a \cdot \Lambda(\mathbf{M}(a), \mathbf{B}),
\end{aligned}
$$

† This part of the argument can equally well be based on the impossibility of determining about two W.F.F. whether they are interconvertible. (Church [3], 363.) 
then $I$ say that $\mathbf{K}_{1}$ is a complete logic formula. $\mathbf{S q}(\mathbf{D}, \mathbf{m}, \mathbf{n})$ is a $\mathrm{C}-\mathbf{K}$ ordinal formula representing $\delta+m \omega+n$, and therefore $\mathrm{Hg}(\mathbf{A}, \mathbf{r}, \mathrm{Sq}(\mathbf{D}))$ represents an ordinal $\zeta_{r}$ which increases steadily with increasing $r$, and tends to the limit $\delta+\omega^{2}$ if $\mathbf{A}$ is dual. Further

$$
\mathrm{Hg}(\mathbf{A}, \mathbf{r}, \mathrm{Sq}(\mathbf{D}))<\mathrm{Hg}\left(\mathbf{A}, S^{\prime}(\mathbf{r}), \mathrm{Sq}(\mathbf{D})\right)
$$

for each positive integer $r$. Therefore $\lambda u f x . u(\lambda y \cdot \mathrm{Hg}(\mathbf{A}, y, \mathrm{Sq}(\mathbf{D})))$ is a C-K ordinal formula and represents the limit of the sequence $\zeta_{1}, \zeta_{2}, \zeta_{3}, \ldots$ This is $\delta+\omega^{2}$ if $\mathbf{A}$ is dual, but a smaller ordinal otherwise. Likewise $\mathbf{M}(\mathbf{A})$ represents $\gamma$ if $\mathbf{A}$ is dual, but is a smaller ordinal otherwise. The formula $\mathbf{B}$ therefore belongs to the extent of $\Lambda(H(\mathbf{M}(\mathbf{A})))$ if and only if $\mathbf{A}$ is dual, and this implies that $\mathbf{K}_{\mathbf{1}}$ is a complete logic formula, as was asserted. But this is impossible and we have the required contradiction.

As a corollary to (A) we see that $\Lambda_{H}$ is incomplete and in fact that the extent of $\Lambda_{H}(\mathrm{Dt})$ contains the extent of $\Lambda_{H}(\Omega)$ for any ordinal formula $\Omega$. This result, suggested to me first by the solution of question (b), may also be obtained more directly. In fact, if a number-theoretic theorem can be proved in any particular $P_{\Omega}$, it can also be proved in $P_{\lambda m u, m(n, I, 4)}$. The formulae describing number-theoretic theorems in $P$ do not involve more than a finite number of types, type 3 being the highest necessary. The formulae describing the number-theoretic theorems in any $P_{\Omega}$ will be obtained by doubling the type subscripts. Now suppose that we have a proof of a number-theoretic theorem $G$ in $P_{\Omega}$ and that the types occurring in the proof are among $0,2,4,6, t_{1}, t_{2}, t_{3}, \ldots$. We may suppose that they have been arranged with all the even types preceding all the odd types, the even types in order of magnitude and the type $2 m-1$ preceding $2 n-1$ if $\Omega(\mathbf{m}, \mathbf{n})$ conv 2 . Now let each $t_{r}$ be replaced by $10+2 v$ throughout the proof of $G$. We thus obtain a proof of $G$ in $P_{\lambda m n .(n, I, 4)}$.

As with problem $(a)$, the solution of problem $(b)$ does not require the use of high ordinals [e.g. if we make the assumption that the extent of $\Lambda(\Omega)$ is a steadily increasing function of the ordinal represented by $\Omega$ we do not have to consider ordinals higher than $\omega+2]$. However, if we restrict what we are to call ordinal formulae in some way, we shall have corresponding modified problems $(a)$ and $(b)$; the solutions will presumably be essentially the same, but will involve higher ordinals. Suppose, for example, that Prod is a W.F.F. with the property that $\operatorname{Prod}\left(\Omega_{1}, \Omega_{2}\right)$ is an ordinal formula representing $a_{1} a_{2}$ when $\Omega_{1}, \Omega_{2}$ are ordinal formulae representing $a_{1}, a_{2}$ respectively, and suppose that we call a W.F.F. a l-ordinal 
formula when it is convertible to the form $\operatorname{Sum}(\operatorname{Prod}(\Omega, D t), \mathbf{P})$, where $\Omega, \mathbf{P}$ are ordinal formulae of which $\mathbf{P}$ represents a finite ordinal. We may define 1-ordinal logics, 1-completeness and 1-invariance in an obvious way, and obtain a solution of problem $(b)$ which differs from the solution in the ordinary case in that the ordinals less than $\omega^{2}$ take the place of the finite ordinals. More generally the cases that I have in mind are covered by the following theorem.

Suppose that we have a class $V$ of formulae representing ordinals in some manner which we do not propose to specify definitely, and a subsct $\dagger$ $U$ of the class $V$ such that:

(i) There is a formula $\Phi$ such that if $T$ enumerates a sequence of mombers of $U$ representing an increasing sequence of ordinals, then $\Phi(T)$ is a member of $U$ representing the limit of the sequence.

(ii) There is a formula $\mathbf{E}$ such that $\mathbf{E}(\mathbf{m}, \mathbf{n})$ is a member of $U$ for each pair of positive integers $m, n$ and, if it represents $\epsilon_{m, n}$, then $\epsilon_{m, n}<\epsilon_{m^{\prime}, u^{\prime}}$ if either $m<m^{\prime}$ or $m=m^{\prime}, n<n^{\prime}$.

(iii) There is a formula $\mathbf{G}$ such that, if $\mathbf{A}$ is a member of $U$, then $\mathbf{G}(\mathbf{A})$ is a member of $U$ representing a larger ordinal than does $\mathbf{A}$, and such that $\mathbf{G}(\mathbf{E}(\mathbf{m}, \mathbf{n}))$ always represents an ordinal not larger than $\epsilon_{m, n+1}$.

We define a $V$-ordinal logic to be a W.F.F. $\Lambda$ such that $\Lambda(A)$ is a logic whenever $\mathbf{A}$ belongs to $V . \quad \Lambda$ is $V$-invariant if the extent of $\Lambda$ (A) depends only on the ordinal represented by $\mathbf{A}$. Then it is not possible for a $V$-ordinal logic $\Lambda$ to be $V$-invariant and have the property that, if $\mathbf{C}_{1}$ represents a greater ordinal than $\mathbf{C}_{\boldsymbol{z}}\left(\mathbf{C}_{1}\right.$ and $\mathbf{C}_{2}$ both being members of $U$ ), then the extent of $\Lambda\left(\mathrm{C}_{1}\right)$ is greater than the extent of $\Lambda\left(\mathrm{C}_{2}\right)$.

We suppose the contrary. Let $\mathbf{B}$ be a formula belonging to the extent of $\Lambda((\Phi(\lambda r . \mathbf{E}(r, 1))))$ but not to the extent of $\Lambda(\Phi(\lambda r . \mathbf{E}(r, 1)))$, and let $\quad \mathbf{K}^{\prime} \rightarrow \lambda a \cdot \Lambda(\mathbf{G}(\boldsymbol{\Phi}(\lambda r \cdot \mathrm{Hg}(a, r, \mathbf{E})), \mathbf{B})$.

Then $\mathbf{K}^{\prime}$ is a complete logic. For

$$
\mathrm{Hg}(\mathbf{A}, \mathbf{r}, \mathbf{E}) \operatorname{conv} \mathbf{E}\left(\mathbf{m}_{\mathbf{r}}, \mathbf{n}_{\mathbf{r}}\right) \text {. }
$$

† The subset $U$ wholly supersedes $V$ in what follows. The introduction of $V$ serves to cimphasise the fact that the set of ordinals represented by members of $U$ may have gaps. 
$\mathbf{E}\left(\mathbf{m}_{\mathbf{r}}, \mathbf{n}_{\mathbf{r}}\right)$ is a sequence of $V$-ordinal formulae representing an increasing sequence of ordinals. Their limit is represented by $\Phi(\lambda r . \mathrm{Hg}(\mathbf{A}, r, \mathbf{E}))$; let us see what this limit is. First suppose that $\mathbf{A}$ is dual : then $m_{r}$ tends to infinity as $r$ tends to infinity, and $\Phi(\lambda r . \mathrm{Hg}(\mathbf{A}, r, \mathbf{E}))$ therefore represents the same ordinal as $\Phi(\lambda r . \mathbf{E}(r, 1))$. In this case we must have

$$
\mathbf{K}^{\prime}(\mathbf{A}) \text { conv } 2 .
$$

Now suppose that $\mathbf{A}$ is not dual: $m_{r}$ is eventually equal to some constant number, $a$ say, and $\Phi(\lambda r . \mathrm{Hg}(\mathbf{A}, r, \mathbf{E}))$ represents the same ordinal as $\Phi(\lambda r . \mathbf{E}(\mathbf{a}, r))$, which is smaller than that represented by $\Phi(\lambda r . \mathbf{E}(r, \mathrm{l})$.$) .$ B cannot therefore belong to the extent of $\boldsymbol{\Lambda}(\mathbf{G}(\boldsymbol{\Phi}(\lambda r . \operatorname{Hg}(\mathbf{A}, r, \mathbf{E}))))$, and $\mathbf{K}^{\prime}(\mathbf{A})$ is not convertible to 2 . We have proved that $\mathbf{K}^{\prime}$ is a complete logic, which is impossible.

This theorem can no doubt be improved in many ways. However, it is sufficiently general to show that, with almost any reasonable notation for ordinals, completeness is incompatible with invariance.

We can still give a certain meaning to the classification into depths with highly restricted kinds of ordinals. Suppose that we take a particular ordinal logic $\Lambda$ and a particular ordinal formula $\Psi$ representing the ordinal a say (preferably a large one), and that we restrict ourselves to ordinal formulae of the form $\operatorname{Inf}(\Psi, a)$. We then have a classification into depths, but the extents of all the logics which we so obtain are contained in the extent of a single logic.

We now attempt a problem of a rather different character, that of the completeness of $\Lambda_{P}$. It is to be expected that this ordinal logic is complete. I cannot at present give a proof of this, but I can give a proof that it is complete as regards a simpler type of theorem than the number-theoretic theorems, viz. those of form " $\theta(x)$ vanishes identically", where $\theta(x)$ is primitive recursive. The proof will have to be much abbreviated since we do not wish to go into the formal details of the system $P$. Also there is a certain lack of definiteness in the problem as at present stated, owing to the fact that the formulae $G, E, M_{P}$ were not completely defined. Our attitude here is that it is open to the sceptical reader to give detailed definitions for these formulae and then verify that the remaining details of the proof can be filled in, using his definition. It is not asserted that these details can be filled in whatever be the definitions of $G, E, M_{P}$ consistent with the properties already required of them, only that they can be filled in with the more natural definitions.

SER. 2. vOL. 45. No. 2242. 
I shall prove the completeness theorem in the following form. If $\mathfrak{B}\left[x_{0}\right]$ is a recursion formula and if $\mathfrak{B}[0], \mathfrak{B}[f 0], \ldots$ are all provable in $P$, then there is a C-K ordinal formula A such that $\left(x_{0}\right) \mathfrak{B}\left[x_{0}\right]$ is provable in the system $P^{\mathrm{A}}$ of logic obtained from $P$ by adjoining as axioms all formulae whose G.R.'s are of the form

$$
\mathbf{A}\left(\lambda m n . m(\varpi(2, n), \varpi(3, n)), K, M_{P}, \mathbf{r}\right)
$$

(provided they represent propositions).

First let us define the formula A. Suppose that $\mathbf{D}$ is a W.F.F. with the property that $\mathbf{D}(\mathbf{n})$ conv 2 if $\mathfrak{B}\left[f^{(n-1)} 0\right]$ is provable in $P$, but $\mathbf{D}(\mathbf{n})$ conv $\mathbf{l}$ if $\sim \mathfrak{B}\left[f^{(n-1)} 0\right]$ is provable in $P$ ( $P$ is being assumed consistent). Let $\Theta$ be defined by

$$
\Theta \rightarrow\{\lambda v u \cdot u(v(v, u))\}(\lambda v u \cdot u(v(v, u))),
$$

and let $\mathrm{Vi}$ be a formula with the properties

$$
\begin{aligned}
& \mathrm{Vi}(2) \operatorname{conv} \lambda u . u(\text { Suc }, U), \\
& \operatorname{Vi}(1) \operatorname{conv} \lambda u . u(I, \Theta(\mathrm{Suc})) .
\end{aligned}
$$

The existence of such a formula is established in Kleene [1], corollary on p. 220. Now put

$$
\begin{aligned}
& \mathbf{A}^{: *} \rightarrow \lambda u f x \cdot u(\lambda y \cdot \operatorname{Vi}(\mathbf{D}(y), y, u, f, x)), \\
& \mathbf{A} \rightarrow \operatorname{Suc}\left(\mathbf{A}^{*}\right) .
\end{aligned}
$$

I assert that $A^{*}, \mathbf{A}$ are $\mathrm{C}-\mathrm{K}$ ordinal formulae whenever it is true that $\mathfrak{B}[0], \mathfrak{S}[f 0], \ldots$ are all provable in $P$. For in this case $\mathbf{A}^{*}$ is $\lambda u f x . u(\mathbf{R})$, where

$$
\mathbf{R} \rightarrow \lambda y \cdot \operatorname{Vi}(\mathbf{D}(y), y, u, f, x),
$$

and then

$\lambda u f x \cdot \mathbf{R}(\mathbf{n}) \operatorname{conv} \lambda u f x \cdot \operatorname{Vi}(\mathbf{D}(\mathbf{n}), \mathbf{n}, u, f, x)$

$$
\begin{aligned}
& \operatorname{conv} \lambda u f x . \operatorname{Vi}(2, \mathbf{n}, u, f, x) \\
& \operatorname{conv} \lambda u f x .\{\lambda n . n(\text { Suc }, U)\}(\mathbf{n}, u, f, x)
\end{aligned}
$$

and

$$
\text { conv } \lambda u f x \cdot \mathbf{n} \text { (Suc, } U, u f, x \text { ), which is a C-K ordinal formula, }
$$

$$
\lambda u f x . S(\mathbf{n}, \operatorname{Suc}, U, u, f, x) \operatorname{conv} \operatorname{Suc}(\lambda u f x \cdot \mathbf{n}(\operatorname{Suc}, U, u, f, x)) .
$$


These relations hold for an arbitrary positive integer $n$ and therefore $A^{*}$ is a $\mathrm{C}-\mathrm{K}$ ordinal formula [condition (9) p. 181]: it follows immediately that $\mathbf{A}$ is also a $\mathrm{C}-\mathrm{K}$ ordinal formula. It remains to prove that $\left(x_{0}\right) \mathfrak{B}\left[x_{0}\right]$ is provable in $P^{\mathrm{A}}$. To do this it is necessary to examine the structure of $\mathbf{A}^{*}$ in the case in which $\left(x_{0}\right) \mathfrak{B}\left[x_{0}\right]$ is false. Let us suppose that $\sim \mathfrak{B}\left[f^{(\alpha-1)} 0\right]$ is true, so that $\mathbf{D}(\mathbf{a})$ conv $\mathbf{1}$, and let us consider $\mathbf{B}$ where

$$
\mathbf{B} \rightarrow \lambda u f x . \operatorname{Vi}(\mathbf{D}(\mathbf{a}), \mathbf{a}, u, f, x) .
$$

If $\dot{A}^{*}$ was a C-K ordinal formula, then $\mathbf{B}$ would be a member of its fundamental sequence; but

$$
\begin{aligned}
& \text { B } \operatorname{conv} \lambda u f x \cdot \operatorname{Vi}(1, \mathbf{a}, u, f, x) \\
& \operatorname{conv} \lambda u f x \cdot\{\lambda u \cdot u(I, \Theta(\text { Suc }))\}(\mathbf{a}, u, f, x) \\
& \operatorname{conv} \lambda u f x \cdot \Theta(\text { Suc }, u, f, x) \\
& \operatorname{conv} \lambda u f x \cdot\{\lambda u \cdot u(\Theta(u))\}(\text { Suc }, u, f, x) \\
& \operatorname{conv} \lambda u f x \cdot \operatorname{Suc}(\Theta(\operatorname{Suc}), u, f, x) \\
& \operatorname{conv} \operatorname{Suc}(\lambda u f x \cdot \Theta(\operatorname{Suc}, u, f, x)) \\
& \operatorname{conv} \operatorname{Suc}(\mathbf{B}) .
\end{aligned}
$$

This, of course, implies that $\mathbf{B}<\mathbf{B}$ and therefore that $\mathbf{B}$ is no C-K ordinal formula. This, although fundamental in the possibility of proving our completeness theorem, does not form an actual step in the argument. Roughly speaking, our argument amounts to this. The relation (9.3) implies that the system $P^{\mathrm{B}}$ is inconsistent and therefore that $P^{\mathrm{A}^{*}}$ is inconsistent and indeed we can prove in $P$ (and a fortiori in $P^{\mathrm{A}}$ ) that $\sim\left(x_{0}\right) \mathfrak{B}\left[x_{0}\right]$ implies the inconsistency of $P^{\mathrm{A}^{*}}$. On the other hand in $P^{\mathrm{A}}$ we can prove the consistency of $P^{\mathrm{A}^{*}}$. The inconsistency of $P^{\mathrm{B}}$ is proved by the Gödel argument. Let us return to the details.

The axioms in $P^{\mathbb{B}}$ are those whose G.R.'s are of the form

$$
\mathbf{B}\left(\lambda m n . m(\varpi(2, n), \varpi(3, n)), K, M_{P}, \mathbf{r}\right) .
$$


When we replace $\mathbf{B}$, by $\operatorname{Suc}(\mathbf{B})$, this becomes

$$
\begin{aligned}
\operatorname{Suc}(\mathbf{B}, \lambda m n . m(\varpi(2, n), & \left.\varpi(3, n)), K, M_{P}, \mathbf{r}\right) \\
& \operatorname{conv} K\left(\mathbf{B}\left(\lambda m n . m(\varpi(2, n), \varpi(3, n)), K, M_{P}, \mathbf{r}\right)\right) \\
& \operatorname{conv} \mathbf{B}\left(\lambda m n . m(\varpi(2, n), \varpi(3, n)), K, M_{P}, \mathbf{p}\right)
\end{aligned}
$$

if $\mathbf{r}$ conv $\mid 2 p+1$,

$$
\operatorname{conv} E\left(\mathbf{B}\left(\lambda m n . m(\varpi(2, n), \varpi(3, n)), K, M_{P}\right), \mathbf{p}\right)
$$

if $\mathbf{r}$ conv $2 \mathbf{p}$.

When we remember the essential property of the formula $E$, we see that the axioms of $P^{\mathbf{B}}$ include all formulae of the form

$$
\left(\exists x_{0}\right) \operatorname{Proof}_{\mathrm{P}}\left[x_{0}, f^{(q)} 0\right] \supset \mathfrak{F},
$$

where $q$ is the G.R. of the formula $\mathfrak{F}$.

Let $b$ be the G.R. of the formula $\mathfrak{A}$.

$$
\sim\left(\exists x_{0}\right)\left(\exists y_{0}\right)\left\{\operatorname{Proof}_{\mathrm{PB}}\left[x_{0}, y_{0}\right] . \mathrm{Sb}\left[z_{0}, z_{0}, y_{0}\right]\right\} .
$$

$\mathrm{Sb}\left[x_{0}, y_{0}, z_{0}\right]$ is a particular recursion formula such that $\mathrm{Sb}\left[f^{(n)} 0, f^{(m)} 0, f^{(n)} 0\right]$ holds if and only if $n$ is the G.R. of the result of substituting $f^{(m)} 0$ for $z_{0}$ in the formula whose G.R. is $l$ at all points where $z_{0}$ is free. Let $p$ be the G.R. of the formula 5 .

$$
\sim\left(\Xi x_{0}\right)\left(\exists y_{0}\right)\left\{\operatorname{Proof}_{\mathrm{P}} \mathrm{B}\left[x_{0}, y_{0}\right] . \operatorname{Sb}\left[f^{(b)} 0, f^{(b)} 0, y_{0}\right]\right\} .
$$

Then we have as an axiom in $P$

$$
\left(\exists x_{0}\right) \operatorname{Proof}_{\mathrm{P}} \mathrm{B}\left[x_{0}, f^{(p)} 0\right] \supset \Subset,
$$

and we can prove in $P^{A}$

$$
\left(x_{0}\right)\left\{\mathrm{Sb}\left[f^{(b)} 0, f^{(b)} 0, x_{0}\right] \equiv x_{0}=f^{(p)} 0\right\},
$$

since $\mathbb{E}$ is the result of substituting $f^{(\iota)} 0$ for $z_{0}$ in $\mathfrak{A}$; hence

$$
\sim\left(\exists y_{0}\right) \operatorname{Proof}_{\mathrm{P}} \mathrm{B}\left[y_{0}, f^{(\nu)} 0\right]
$$

is provable in $P$. Using (9.4) again, we see that $\mathbb{E}$ can be proved in $P^{\mathbf{B}}$. But, if we can prove $\mathfrak{E}$ in $P^{B}$, then we can prove its provability in $P^{B}$, the 
proof being in $P$; i.e. we can prove

$$
\left(\exists x_{0}\right) \operatorname{Proof}_{\mathrm{PB}}\left[x_{0}, f^{(p)} 0\right]
$$

in $P$ (since $p$ is the G.R. of $\Subset$ ). But this contradicts $(9.5)$, so that, if

$$
\sim \mathfrak{B}\left[f^{(a-1)} 0\right]
$$

is true, we can prove a contradiction in $P^{\mathrm{B}}$ or in $P^{\mathrm{A}^{*}}$. Now I assert that the whole argument up to this point can be carried through formally in the system $P$, in fact, that, if $c$ is the G.R. of $\sim(0=0)$, then

$$
\sim\left(x_{0}\right) \mathfrak{B}\left[x_{0}\right) \supset\left(\exists v_{0}\right) \operatorname{Proof}_{\mathrm{P}^{*}}\left[v_{0}, f^{(c)} 0\right]
$$

is provable in $P$. I shall not attempt to give any more detailed proof of this assertion.

The formula

$$
\left(\exists x_{0}\right) \operatorname{Proof}_{\mathrm{PA}^{*}}\left[x_{0}, f^{(c)} 0\right] \supset \sim(0=0)
$$

is an axiom in $P^{\mathrm{A}}$. Combining (9.6), (9.7) we obtain $\left(x_{0}\right) \mathfrak{B}\left[x_{0}\right]$ in $P^{\mathrm{A}}$.

This completeness theorem as usual is of no value. Although it shows, for instance, that it is possible to prove Fermat's last theorem with $\Lambda_{P}$ (if it is true) yet the truth of the theorem would really be assumed by taking a certain formula as an ordinal formula.

That $\Lambda_{P}$ is not invariant may be proved easily by our general theorem; alternatively it follows from the fact that, in proving our partial completeness theorem, we never used ordinals higher than $\omega+1$. This fact can also be used to prove that $\Lambda_{P}$ is not $\mathrm{C}-\mathrm{K}$ invariant up to $\omega+2$.

\section{The continuum hypothesis. A digression.}

The methods of $\S 9$ may be applied to problems which are constructive analogues of the continuum hypothesis problem. The continuum hypothesis asserts that $2 N_{0}=N_{1}$, in other words that, if $\omega_{1}$ is the smallest ordinal $a$ greater than $\omega$ such that a series with order type $a$ cannot be put into one-one correspondence with the positive integers, then the ordinals less than $\omega_{1}$ can be put into one-one correspondence with the subsets of the positive integers. To obtain a constructive analogue of this proposition we may replace the ordinals less than $\omega_{1}$ either by the ordinal formulae, or by the ordinals represented by them; we may replace the subsets of the positive integers either by the computable sequences of figures 0,1 , or by the description numbers of the machines which compute these sequences. In the manner in which the correspondence is to be set up there is also more than one possibility. Thus, even when we use only 
one kind of ordinal formula, there is still great ambiguity concerning what the constructive analogue of the continuum hypothesis should be. I shall prove a single result in this connectiont. A number of others may be proved in the same way.

We ask "Is it possible to find a computable function of ordinal formulae determining a one-one correspondence between the ordinals represented by ordinal formulae and the computable sequences of figures 0,1 ?" More accurately, "Is there a formula $F$ such that if $\Omega$ is an ordinal formula and $n$ a positive integer then $\mathbf{F}(\Omega, \mathbf{n})$ is convertible to $l$ or to 2 , and such that $\mathbf{F}(\boldsymbol{\Omega}, \mathbf{n}) \operatorname{conv} \mathbf{F}\left(\Omega^{\prime}, \mathbf{n}\right)$ for each positive integer $n$, if and only if $\Omega$ and $\boldsymbol{\Omega}^{\prime}$ represent the same ordinal?" The answer is "No", as will be seen to be a consequence of the following argument: there is no formula $\mathbf{F}$ such that $F(\Omega)$ enumerates one sequence of integers (each being 1 or 2 ) when $\Omega$ represents $\omega$ and enumerates another sequence when $\Omega$ represents 0 . If there is such an $\mathbf{F}$, then there is an $a$ such that $\mathbf{F}(\Omega$, a) $\operatorname{conv}(\mathrm{Dt}, \mathbf{a})$ if $\Omega$ represents $\omega$ but $\mathbf{F}(\Omega, a)$ and $\mathbf{F}(\mathrm{Dt}, \mathbf{a})$ are convertible to different integers (l or 2 ) if $\Omega$ represents 0 . To obtain a contradiction from this we introduce a W.F.F. Gm not unlike $\mathrm{Mg}$. If the machine $A$ whose D.N. is $n$ has printed 0 by the time the $m$-th complete configuration is reached then

$$
\mathrm{Gm}(\mathbf{n}, \mathbf{m}) \text { convì } m n \cdot m(n, I, 4) \text {; }
$$

otherwise $\operatorname{Gm}(\mathbf{n}, \mathbf{m}) \operatorname{conv} \lambda p q \cdot \operatorname{Al}(4(P, 2 p+2 q), 3,4)$. Now consider $\mathbf{F}(\mathrm{Dt}, \mathbf{a})$ and $\mathbf{F}(\operatorname{Lim}(\operatorname{Gm}(\mathbf{n})), \mathbf{a})$. If .l never prints $0, \operatorname{Lim}(\operatorname{Gm}(\mathbf{n}))$ represents the ordinal $\omega$. Otherwise it represents 0 . Consequently these two formulae are convertible to one another if and only if 4 never prints 0 . This gives us a means of determining about any machine whether it ever prints 0 , which is impossible.

Results of this kind have of course no real relevance for the classical continuum hypothesis.

\section{The purpose of ordinal logics.}

Mathematical reasoning may be regarded rather schematically as the exercise of a combination of two faculties $\ddagger$, which we may call intuition and ingenuity. The activity of the intuition consists in making spontaneous judgments which are not the result of conscious trains

† A suggestion to consider this problem came to me indirectly from $F$. Bernstein. A related problem was suggested by $P$. Bernays.

$\ddagger$ We are leaving out of account that most important faculty which distinguishes topics of interest from others; in fact, we are regarding the function of the mathematician as simply to determine the truth or falsity of propositions. 
of reasoning. These judgments are often but by no means invariably correct (leaving aside the question what is meant by "correct"). Often it is possible to find some other way of verifying the correctness of an intuitive judgment. We may, for instance, judge that all positive integers are uniquely factorizable into primes; a detailed mathematical argument leads to the same result. This argument will also involve intuitive judgments, but they will be less open to criticism than the original judgment about factorization. I shall not attempt to explain this idea of "intuition" any more explicitly.

The exercise of ingenuity in mathematics consists in aiding the intuition through suitable arrangements of propositions, and perhaps geometrical figures or drawings. It is intended that when these are really well arranged the validity of the intuitive steps which are required cannot seriously be doubted.

The parts played by these two faculties differ of course from occasion to occasion, and from mathematician to mathematician. This arbitrariness can be removed by the introduction of a formal logic. The necessity for using the intuition is then greatly reduced by setting down formal rules for carrying out inferences which are always intuitively valid. When working with a formal logic, the idea of ingenuity takes a more definite shape. In general a formal logic, will be framed so as to admit a considerable variety of possible steps in any stage in a proof. Ingenuity will then determine which steps are the more profitable for the purpose of proving a particular proposition. In pre-Gödel times it was thought by some that it would probably be possible to carry this programme to such a point that all the intuitive judgments of mathematics could be replaced by a finite number of these rules. The necessity for intuition would then be entirely eliminated.

In our discussions, however, we have gone to the opposite extreme and eliminated nct intuition but ingenuity, and this in spite of the fact that our aim has been ' $n$ much the same direction. We have been trying to see how far it is possible to eliminate intuition, and leave only ingenuity. We do not mind how much ingenuity is required, and therefore assume it to be available in unlimited supply. In our metamathematical discussions we actually express this assumption rather differently. We are always able to obtain from the rules of a formal logic a method of enumerating the propositions proved by its means. We then imagine that all proofs take the form of a search through this enumeration for the theorem for which a proof is desired. In this way ingenuity is replaced by patience. In these heuristic discussions, however, it is better not to make this reduction. 
In consequence of the impossibility of finding a formal logic which wholly eliminates the necessity of using intuition, we naturally turn to "nonconstructive" systems of logic with which not all the steps in a proof are mechanical, some being intuitive. An example of a non-constructive logic is afforded by any ordinal logic. When we have an ordinal logic, we are in a position to prove number-theoretic theorems by the intuitive steps of recognizing formulae as ordinal formulae, and the mechanical steps of carrying out conversions. What properties do we desire a non-constructive logic to have if we are to make use of it for the expression of mathematical proofs? We want it to show quite clearly when a step makes use of intuition, and when it is purely formal. The strain put on the intuition should be a minimum. Most important of all, it must be beyond all reasonable doubt that the logic leads to correct results whenever the intuitive steps are correct $\uparrow$. It is also desirable that the logic shall be adequate for the expression of number-theoretic theorems, in order that it may be used in metamathematical discussions (cf. §5).

Of the particular ordinal logics that we have discussed, $\Lambda_{H}$ and $\Lambda_{P}$ certainly will not satisfy us. In the case of $\Lambda_{H}$ we are in no better position than with a constructive logic. In the case of $\Lambda_{P}$ (and for that matter also $\Lambda_{H}$ ) we are by no means certain that we shall never obtain any but true results, because we do not know whether all the number-theoretic theorems provable in the system $P$ are true. To take $\Lambda_{P}$ as a fundamental non-constructive logic for metamathematical arguments would be most unsound. There remains the system of Church which is free from these objections. It is probably complete (although this would not necessarily mean much) and it is beyond reasonable doubt that it always leads to correct results $\neq$. In the next section I propose to describe another ordinal logic, of a very different type, which is suggested by the work of Gentzen and which should also be adequate for the formalization of number-theoretic theorems. In particular it should be suitable for proofs of metamathematical theorems (cf. §5).

$\dagger$ This requirement is very vague. It is not of course intended that the criterion of the correctness of the intuitive steps be the correctness of the final result. The meaning becomes clearer if each intuitive step is regarded as a judgment that a particular proposition is true. In the case of an ordinal logic it is always a judgment that a formula is an ordinal formula, and this is equivalent to judging that a number-theoretic proposition is true. In this case then the requirement is that the reputed ordinal logic is an ordinal logic.

† This ordinal logic arises from a certain system $C_{0}$ in essentially the same way as $\Lambda_{P}$ arose from $P$. By an argument similar to one occurring in $\$ 8$ we can show that the ordinal logic leads to correct results if and only if $C_{0}$ is valid; the validity of $C_{0}$ is proved in Church [1], making use of the results of Church and Rosser [1]. 


\section{Gentzen type ordinal logics.}

In proving the consistency of a certain system of formal logic Gentzen (Gentzen [1]) has made use of the principle of transfinite induction for ordinals less than $\epsilon_{0}$, and has suggested that it is to be expected that transfinite induction carried sufficiently far would suffice to solve all problems of consistency. Another suggestion of basing systems of logic on transfinite induction has been made by Zermelo (Zermelo [1]). In this section I propose to show how this method of proof may be put into the form of a formal (non-constructive) logic, and afterwards to obtain from it an ordinal logic.

We can express the Gentzen method of proof formally in this way. Let us take the system $P$ and adjoin to it an axiom $\mathfrak{A} \Omega$ with the intuitive meaning that the W.F.F. $\Omega$ is an ordinal formula, whenever we feel certain that $\Omega$ is an ordinal formula. This is a non-constructive system of logic which may easily be put into the form of an ordinal logic. By the method of $\S 6$ we make correspond to the system of logic consisting of $P$ with the axiom $\mathfrak{A}_{\Omega}$ adjoined a logic formula $L_{\Omega}: L_{\Omega}$ is an effectively calculable function of $\Omega$, and there is therefore a formula $\Lambda_{G}{ }^{1}$ such that $\Lambda_{G}{ }^{1}(\Omega) \operatorname{conv} L_{\Omega}$ for each formula $\Omega$. $\quad \Lambda_{G}{ }^{1}$ is certainly not an ordinal logic unless $P$ is valid, and therefore consistent. This formalization of Gentzen's idea would therefore not be applicable for the problem with which Gentzen himself was concerned, for he was proving the consistency of a system weaker than $P$. However, there are other ways in which the Gentzen method of proof can be formalized. I shall explain one, beginning by describing a certain logical calculus.

The symbols of the calculus are $f, x,{ }^{1},{ }_{1}, 0, S, R, \Gamma, \Delta, E, \mid, \odot, !,($, ), =, and the comma ",". For clarity we shall use various sizes of brackets $($,$) in the following. We use capital German letters to stand for variable$ or undetermined sequences of these symbols.

It is to be understood that the relations that we are about to define hold only when compelled to do so by the conditions that we lay down. The conditions should be taken together as a simultaneous inductive definition of all the relations involved.

Suffixes.

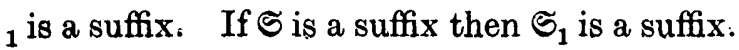

\section{Indices.}

1 is an index. If $\mathfrak{\Im}$ is an index then $\mathfrak{\Im}^{1}$ is an index.

Numerical variables.

If $\subseteq$ is a suffix then $x \subseteq$ is a numerical variable. 
Functional variables.

If $\mathfrak{S}$ is a suffix and $\mathfrak{\Im}$ is an index, then $f \subseteq \Im$ is a functional variable of index $\mathfrak{\Im}$.

Arguments.

$($,$) is an argument of index { }^{\mathbf{1}}$. If $(\mathfrak{I})$ is an argument of index $\mathfrak{\Im}$ and $\mathfrak{T}$ is a term, then $(\mathfrak{A} \mathfrak{Z}$,$) is an argument of index \mathfrak{J}^{1}$.

Numerals.

0 is a numeral.

If $\mathfrak{N}$ is a numeral, then $S(, \mathfrak{N}$,) is a numeral.

In metamathematical statements we shall denote the numeral in which $S$ occurs $r$ times by $S^{(r)}(, 0$,$) .$

Expressions of a given index.

A functional variable of index $\mathfrak{\Im}$ is an expression of index $\Im$.

$R, S$ are expressions of index ${ }^{111},{ }^{11}$ respectively.

If $\Re$ is a numeral, then it is also an expression of index ${ }^{1}$.

Suppose that $\mathfrak{S}$ is an expression of index $\mathfrak{\Im}, \mathfrak{H}$ one of index $\mathfrak{J}^{1}$ and $\mathfrak{N}$ one of index $\mathfrak{\Im}^{111}$; then $(\Gamma \mathfrak{S})$ and $(\Delta \mathfrak{G})$ are expressions of index $\Im$, while $(E \mathbb{G})$ and $(\mathfrak{S} \mid \mathfrak{H})$ and $(\mathscr{S} \odot \mathfrak{R})$ and $(\mathfrak{S} ! \mathfrak{h} ! \mathfrak{R})$ are expressions of index $\mathfrak{S}^{1}$.

Function constants.

An expression of index $\Im$ in which no functional variable occurs is a function constant of index $\Im$. If in addition $R$ does not occur, the expression is called a primitive function constant.

Terms.

0 is a term.

Every numerical variable is a term.

If $\mathfrak{S}$ is an expression of index $\mathfrak{I}$ and $(\mathfrak{A})$ is an argument of index $\mathfrak{\Im}$, then $\mathfrak{G}(\mathfrak{A})$ is a term.

\section{Equations.}

If $\mathfrak{I}$ and $\mathfrak{X}^{\prime}$ are terms, then $\mathfrak{x}=\mathfrak{X}^{\prime}$ is an equation.

Provable equations.

We define what is meant by the provable equations relative to a given set of equations as axioms. 
(a) The provable equations include all the axioms. The axioms are of the form of equations in which the symbols $\Gamma, \Delta, E, \mid, \odot, !$ do not appear.

(b) If $\mathfrak{F}$ is an expression of index $\mathfrak{J}^{11}$ and $(\mathfrak{H})$ is an argument of index $\mathfrak{\Im}$ then

$$
(\Gamma \mathfrak{G})\left(\mathfrak{H} x_{1}, x_{11},\right)=\mathfrak{G}\left(\mathfrak{2} x_{11}, x_{1}\right)
$$

is a provable equation.

(c) If $\circlearrowleft$ is an expression of index $\mathfrak{\Im}^{1}$, and $(\mathfrak{H})$ is an argument of index $\mathfrak{\Im}$, then

$$
(\Delta \mathfrak{S})\left(\mathfrak{A}\left(x_{1},\right)=\mathfrak{G}\left(, x_{1} \mathfrak{A}\right)\right.
$$

is a provable equation. then

$(d)$ If $\mathfrak{G}$ is an expression of index $\mathfrak{\Im}$, and $(\mathfrak{H})$ is an argument of index $\mathfrak{\Im}$,

$$
\left(E(\mathfrak{S})\left(\mathfrak{A} x_{1},\right)=\mathfrak{S}(\mathfrak{H})\right.
$$

is a provable equation.

(e) If $\mathfrak{B}$ is an expression of index $\mathfrak{S}$ and $\mathfrak{S}$ is one of index $\mathfrak{S}^{1}$, and $(\mathfrak{I})$ is an argument of index $\mathfrak{s}$, then

$$
(\mathbb{S} \mid \mathfrak{S})(\mathfrak{A})=\mathfrak{J}(\mathfrak{A}(\mathfrak{S}(\mathfrak{H}),)
$$

is a provable equation.

$(f)$ If $\mathfrak{N}$ is an expression of index ${ }^{1}$, then $\mathfrak{N}()=,\mathfrak{N}$ is a provable equation.

$(g)$ If $\mathfrak{S}$ is an expression of index $\mathfrak{\Im}$ and $\mathfrak{R}$ one of index $\mathfrak{\Im} 111$, and $(\mathfrak{A})$ an argument of index $\mathfrak{S}^{1}$, then

$$
(\mathfrak{S} \odot \mathfrak{R})(\mathfrak{L} 0,)=\mathfrak{G}(\mathfrak{H})
$$

and

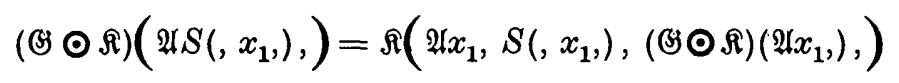

are provable equations. If in addition $\mathfrak{F}$ is an expression of index $\mathfrak{I}^{1}$ and

$$
R\left(, \mathfrak{S}\left(\mathfrak{H} S\left(, x_{1},\right),\right), x_{1},\right)=0
$$

is provable, then

and

$$
(\mathbb{S} ! \mathfrak{R} ! \mathfrak{H})(\mathfrak{H} 0,)=\mathbb{G}(\mathfrak{H})
$$

$$
\begin{aligned}
& (\mathbb{S} ! \mathfrak{R !} \mathfrak{F})\left(\mathfrak{A S}\left(, x_{1},\right),\right) \\
& =\mathfrak{K}\left(\left(\mathfrak{H} \mathfrak{H}\left(\mathfrak{H} S\left(, x_{1},\right)\right), S\left(, x_{1},\right),(\mathfrak{S} ! \mathfrak{H} ! \mathfrak{F})\left(\mathfrak{H} \mathfrak{T}\left(\mathfrak{H} S\left(, x_{1},\right),\right),\right)\right)\right)
\end{aligned}
$$

are provable. 
(h) If $\mathfrak{Z}=\mathfrak{I}^{\prime}$ and $\mathfrak{U}=\mathfrak{U}^{\prime}$ are provable, where $\mathfrak{Z}, \mathfrak{I}^{\prime}, \mathfrak{U}$ and $\mathfrak{U}^{\prime}$ are terms, then $\mathfrak{u}^{\prime}=\mathfrak{U}$ and the result of substituting $\mathfrak{u}^{\prime}$ for $\mathfrak{l}$ at any particular occurrence in $\mathfrak{Z}=\mathfrak{I}^{\prime}$ are provable equations.

(i) The result of substituting any term for a particular numerical variable throughout a provable equation is provable.

(j) Suppose that $\left(\mathfrak{G}, \mathfrak{G}^{\prime}\right.$ are expressions of index $\mathfrak{J}^{1}$, that $(\mathfrak{2})$ is an argument of index $\Im$ not containing the numerical variable $\mathfrak{X}$ and that $\mathfrak{G}(\mathfrak{L} 0)=,\mathscr{G}^{\prime}(\mathfrak{L} 0$,$) is provable. Also suppose that, if we add$

$$
\mathfrak{G S}(\mathfrak{A Z},)=\mathfrak{G S}^{\prime}(\mathfrak{A X},)
$$

to the axioms and restrict $(i)$ so that it can never be applied to the numerical variable $\mathfrak{X}$, then

$$
\mathfrak{S B}(\mathfrak{I} S(, \mathfrak{X},),)=\mathfrak{S}^{\prime}(\mathfrak{H} S(, \mathfrak{X}),)
$$

becomes a provable equation; in the hypothetical proof of this equation this rule $(j)$ itself may be used provided that a different variable is chosen to take the part of $\mathfrak{x}$.

Under these conditions $\mathfrak{S}(\mathfrak{A X})=,\mathfrak{S}^{\prime}(\mathfrak{A X}$,$) is a provable equation.$

(k) Suppose that $\mathfrak{G}, \mathfrak{S}^{\prime}, \mathfrak{S}$ are expressions of index $\mathfrak{S}^{1}$, that $(\mathfrak{H})$ is an argument of index $\mathfrak{\Im}$ not containing the numerical variable $\mathfrak{F}$ and that

$$
\mathfrak{G}\left(\mathfrak{L}(0,)=\mathfrak{G J}^{\prime}(\mathfrak{H} 0,) \quad \text { and } \quad R(, \mathfrak{S}(\mathfrak{H} S(, \mathfrak{X},),), S(, \mathfrak{X},),)=0\right.
$$

are provable equations. Suppose also that, if we add

$$
\text { G) }(\mathfrak{A} \mathfrak{T}(\mathfrak{H} S(, \mathfrak{X},),))=\mathfrak{G}^{\prime}(\mathfrak{H} \mathfrak{S}(\mathfrak{A} S(, \mathfrak{X},),))
$$

to the axioms, and again restrict $(i)$ so that it does not apply to $\mathfrak{X}$, then

$$
\mathfrak{G}(\mathfrak{A Z},)=\mathfrak{S}^{\prime}(\mathfrak{H} \mathfrak{X},)
$$

becomes a provable equation; in the hypothetical proof of (12.1) the rule $(k)$ may be used if a different variable takes the part of $\mathfrak{X}$.

Under these conditions (12.1) is a provable equation.

We have now completed the definition of a provable equation relative to a given set of axioms. Next we shall show how to obtain an ordinal logic from this calculus. The first step is to set up a correspondence between some of the equations and number-theoretic theorems, in other words to show how they can be interpreted as number-theoretic theorems. 
Let $\$$ be a primitive function constant of index ${ }^{111}$. (S) describes a certain primitive recursive function $\phi(m, n)$, determined by the condition that, for all natural numbers $m, n$, the equation

$$
\mathbb{S}\left(, S^{(m)}(, 0,), S^{(n)}(, 0,),\right)=S^{(\phi(m, n))}(, 0,)
$$

is provable without using the axioms $(a)$. Suppose also that $\sqrt{5}$ is an expression of index $\Im$. Then to the equation

$$
\text { (3) }\left(, x_{1}, \mathfrak{S}\left(, x_{1},\right),\right)=0
$$

we make correspond the number-theoretic theorem which asserts that for each natural number $m$ there is a natural number $n$ such that $\phi(m, n)=0$. ('The circumstance that there is more than one equation to represent each number-theoretic theorem could be avoided by a trivial but inconvenient modification of the calculus.)

Now let us suppose that some definite method is chosen for describing the sets of axioms by means of positive integers, the null set of axioms being described by the integer 1. By an argument used in $\S 6$ there is a W.F.F. $\Sigma$ such that, if $r$ is the integer describing a set $A$ of axioms, then $\Sigma(\mathbf{r})$ is a logic formula enabling us to prove just those number-theoretic theorems which are associated with equations provable with the above described calculus, the axioms being those described by the number $r$.

I explain two ways in which the construction of the ordinal logic may be completed.

In the first method we make use of the theory of general recursive functions (Kleene [2]). Let us consider all equations of the form

$$
R\left(, S^{(m)}(, 0,), S^{(n)}(, 0,),\right)=S^{(p)}(, 0,)
$$

which are obtainable from the axioms by the use of rules $(h),(i)$. It is a consequence of the theorem of equivalence of $\lambda$-definable and general recursive functions (Kleene [3]) that, if $r(m, n)$ is any $\lambda$-definable function of two variables, then we can choose the axioms so that (12.2) with $p=r(m, n)$ is obtainable in this way for each pair of natural numbers $m, n$, and no equation of the form

$$
S^{(m)}(, 0,)=S^{(n)}(, 0,) \quad(m \neq n)
$$

is obtainable. In particular, this is the case if $r(m, n)$ is defined by the condition that

$$
\begin{gathered}
\Omega(\mathbf{m}, \mathbf{n}) \operatorname{conv} S(\mathbf{p}) \text { implies } p=r(m, n), \\
r(0, n)=1, \text { all } n>0, \quad r(0,0)=2,
\end{gathered}
$$


where $\Omega$ is an ordinal formula. There is a method for obtaining the axioms given the ordinal formula, and consequently a formula Rec such that, for any ordinal formula $\Omega, \operatorname{Rec}(\Omega)$ conv $\mathbf{m}$, where $m$ is the integer describing the set of axioms corresponding to $\Omega$. Then the formula

$$
\Lambda_{G}{ }^{2} \rightarrow \lambda w . \Sigma(\operatorname{Rec}(w))
$$

is an ordinal logic. Let us leave the proof of this aside for the present.

Our second ordinal logic is to be constructed by a method not unlike the one which we used in constructing $\Lambda_{p}$. We begin by assigning ordinal formulae to all sets of axioms satisfying certain conditions. For this purpose we again consider that part of the calculus which is obtained by restricting "expressions" to be functional variables or $R$ or $S$ and restricting the meaning of "term" accordingly; the new provable equations are given by conditions $(a),(h),(i)$, together with an extra condition $(l)$.

(l) The equation

$$
R\left(, 0, S\left(, x_{1}\right),\right)=0
$$

is provable.

We could design a machine which would obtain all equations of the form (12 . 2), with $m \neq n$, provable in this sense, and all of the form (12.3), except that it would cease to obtain any more equations when it had once obtained one of the latter "contradictory" equations. From the description of the machine we obtain a formula $\Omega$ such that

$$
\Omega(\mathbf{m}, \mathbf{n}) \text { conv } 2 \text { if } \quad R\left(, S^{(m-1)}(, 0,), S^{(n-1)}(, 0,),\right)=0
$$

is obtained by the machine,

$$
\Omega(\mathbf{m}, \mathbf{n}) \text { conv } 1 \text { if } \quad R\left(, S^{(n-1)}(, 0,), S^{(m-1)}(, 0,),\right)=0
$$

is obtained by the machine, and

$$
\Omega(\mathbf{m}, \mathbf{m}) \text { conv } 3 \text { always. }
$$

The formula $\Omega$ is an effectively calculable function of the set of axioms, and therefore also of $m$ : consequently there is a formula $M$ such that $M(\mathbf{m})$ conv $\Omega$ when $m$ describes the set of axioms. Now let $\mathrm{Cm}$ be a formula such that, if $b$ is the G.R. of a formula $M(\mathbf{m})$, then $\mathrm{Cm}(b)$ conv $\mathbf{m}$, but otherwise $\mathrm{Cm}(b)$ conv 1 . Let

$$
\Lambda_{G}{ }^{3} \rightarrow \lambda w a . \Gamma(\lambda n . \Sigma(\operatorname{Cm}(\operatorname{Tn}(w, n)), a) .
$$


Then $\Lambda_{G}{ }^{3}(\Omega, \mathbf{A})$ conv 2 if and only if $\Omega$ conv $M(\mathrm{~m})$, where $m$ describes a set of axioms which, taken with our calculus, suffices to prove the equation which is, roughly speakịng, equivalent to " $A$ is dual". To prove that $\Lambda_{G}{ }^{3}$ is an ordinal logic, it is sufficient to prove that the calculus with the axioms described by $m$ proves only true number-theoretic theorems when $\Omega$ is an ordinal formula. This condition on $m$ may also be expressed in this way. Let us put $m \ll n$ if we can prove $R\left(, S^{(m)}(, 0),, S^{(n)}(, 0),\right)=0$ with $(a)$, $(h),(i),(l)$ : the condition is that $m \ll n$ is a well-ordering of the natural numbers and that no contradictory equation (12.3) is provable with the same rules $(a),(h),(i),(l)$. Let us say that such a set of axioms is admissible. $\Lambda_{G i}{ }^{3}$ is an ordinal logic if the calculus leads to none but true number-theoretic theorems when an admissible set of axioms is used.

In the case of $\Lambda_{G}{ }^{2}, \operatorname{Rec}(\Omega)$ describes an admissible set of axioms whenever $\Omega$ is an ordinal formula. $\Lambda_{G}{ }^{2}$ therefore is an ordinal logic if the calculus leads to correct results when admissible axioms are used.

To prove that admissible axioms have the required property, I do not attempt to do more than show how interpretations can be given to the equations of the calculus so that the rules of inference $(a)-(k)$ become intuitively valid methods of deduction, and so that the interpretation agrees with our convention regarding number-theoretic theorems.

Each expression is the name of a function, which may be only partially defined. The expression $S$ corresponds simply to the successor function. If $\&$ is either $R$ or a functional variable and has $p+1$ symbols in its index, then it corresponds to a function $g$ of $p$ natural numbers defined as follows. If

$$
\left.\mathfrak{S}\left(, S^{\left(r_{1}\right)}(, 0,), S^{\left(r_{2}\right)}(, 0,), \ldots, S^{\left(r_{p}\right)}(, 0,)\right)\right)=S^{(l)}(, 0,)
$$

is provable by the use of $(a),(h),(i),(l)$ only, then $g\left(r_{1}, r_{2}, \ldots, r_{p}\right)$ has the value $p$. It may not be defined for all arguments, but its value is always unique, for otherwise we could prove a "contradictory" equation and $M(\mathbf{m})$ would then not be an ordinal formula. The functions corresponding to the other expressions are essentially defined by $(b)-(f)$. For example, if $g$ is the function corresponding to $\mathbb{B}$ and $g^{\prime}$ that corresponding to $(\Gamma(\mathcal{G})$, then

$$
g^{\prime}\left(r_{1}, r_{2}, \ldots, r_{p}, l, m\right)=g\left(r_{1}, r_{2}, \ldots, r_{p}, m, l\right) .
$$

The values of the functions are clearly unique (when defined at all) if given by one of $(b)-(e)$. The case $(f)$ is less obvious since the function defined appears also in the definiens. I do not treat the case of $(\circlearrowleft \odot \odot \Re)$, since this is the well-known definition by primitive recursion, but I shall show that the values of the function corresponding to $(\mathbb{S} ! \mathfrak{R} ! \mathfrak{S})$ are unique. Without loss of generality we may suppose that $(\mathfrak{A})$ in $(f)$ is of index ${ }^{1}$. We have 
then to show that, if $h(m)$ is the function corresponding to $\mathfrak{T}$ and $r(m, n)$ that corresponding to $R$, and $k(u, v, w)$ is a given function and $a$ a given natural number, then the equations

$$
\begin{aligned}
& l(0)=a, \\
& l(m+1)=k(h(m+1), m+1, l(h(m+1)))
\end{aligned}
$$

do not ever assign two different values for the function $l(m)$. Consider those values of $r$ for which we obtain more than one value of $l(r)$, and suppose that there is at least one such. Clearly 0 is not one, for $l(0)$ can be defined only by $(a)$. Since the relation $\ll$ is a well ordering, there is an integer $r_{0}$ such that $r_{0}>0, l\left(r_{0}\right)$ is not unique, and if $s \neq r_{0}$ and $l(s)$ is not unique then $r_{0} \ll s$. We may put $s=h\left(r_{0}\right)$, for, if $l\left(h\left(r_{0}\right)\right)$ were unique, then $l\left(r_{0}\right)$, defined by $(\beta)$, would be unique. But $r\left(h\left(r_{0}\right), r_{0}\right)=0$ i.e. $s \ll r_{0}$. There is, therefore, no integer $r$ for which we obtain more than one value for the function $l(r)$.

Our interpretation of expressions as functions gives us an immediate interpretation for equations with no numerical variables. In general we interpret an equation with numerical variables as the (infinite) conjunction of all equations obtainable by replacing the variables by numerals. With this interpretation $(h),(i)$ are seen to be valid methods of proof. In $(j)$ the provability of

$$
\text { (j) }\left(\mathfrak{A} S\left(, x_{1},\right),\right)=\mathfrak{G J}^{\prime}\left(\mathfrak{A} S\left(, x_{1},\right),\right)
$$

when $\mathfrak{O}\left(\mathfrak{H} x_{1},\right)=\mathfrak{S}^{\prime}\left(\mathfrak{H} x_{1},\right)$ is assumed to be interpreted as meaning that the implication between these equations holds for all substitutions of numerals for $x_{1}$. To justify this, one should satisfy oneself that these implications always hold when the hypothetical proof can be carried out. The rule of procedure $(j)$ is now seen to be simply mathematical induction. The rulo $(k)$ is a form of transfinite induction. In proving the validity of $(k)$ we may again suppose $(\mathfrak{I})$ is of index ${ }^{1}$. Let $r(m, n), g(m), g_{1}(m), h(n)$ be the functions corresponding respectively to $R, \mathfrak{J}, \mathfrak{S}^{\prime}, \mathfrak{J}$. We shall prove that, if $g(0)=g^{\prime}(0)$ and $r(h(n), n)=0$ for each positive integer $n$ and if $g(n+1)=g^{\prime}(n+1)$ whenever $g(h(n+1))=g^{\prime}(h(n+1))$, then $g(n)=g^{\prime}(n)$ for each natural number $n$. We consider the class of natural numbers for which $g(n)=g^{\prime}(n)$ is not true. If the class is not void it has a positive member $n_{0}$ which precedes all other members in the well ordering $\ll$. But $h\left(n_{0}\right)$ is another member of the class, for otherwise we should have

$$
g\left(h\left(n_{0}\right)\right)=g^{\prime}\left(h\left(n_{0}\right)\right)
$$


and therefore $g\left(n_{0}\right)=g^{\prime}\left(n_{0}\right)$, i.e. $n_{0}$ would not be in the class. This implies $n_{0} \ll h\left(n_{0}\right)$ contrary to $r\left(h\left(n_{0}\right), n_{0}\right)=0$. The class is therefore void.

It should be noticed that we do not really need to make use of the fact that $\Omega$ is an ordinal formula. It suffices that $\Omega$ should satisfy conditions $(a)-(e)$ (p. 179) for ordinal formulae, and in place of $(f)$ satisfy $\left(f^{\prime}\right)$.

$\left(f^{\prime}\right)$ There is no formula $\mathbf{T}$ such that $\mathbf{T}(\mathbf{n})$ is convertible to a formula representing a positive integer for each positive integer $n$, and such that $\Omega(\mathbf{T}(\mathbf{n}), \mathbf{n})$ conv 2 , for each positive integer $n$ for which $\Omega(\mathbf{n}, \mathbf{n})$ conv 3 .

The problem whether a formula satisfies conditions $(a)-(e),\left(f^{\prime}\right)$ is number-theoretic. If we use formulae satisfying these conditions instead of ordinal formulae with $\Lambda_{G}{ }^{2}$ or $\Lambda_{G}{ }^{3}$, we have a non-constructive logic with certain advantages over ordinal logics. The intuitive judgments that must be made are all judgments of the truth of number theoretic-theorems. We have seen in $\S 9$ that the connection of ordinal logics with the classical theory of ordinals is quite superficial. There seem to be good reasons, therefore, for giving attention to ordinal formulae in this modified sense.

The ordinal logic $\Lambda_{G}{ }^{3}$ appears to be adequate for most purposes. It should, for instance, be possible to carry out Gentzen's proof of consistency of number theory, or the proof of the uniqueness of the normal form of a well-formed formula (Church and Rosser [1]) with our calculus and a fairly simple set of axioms. How far this is the case can, of course, only be determined by experiment.

One would prefer a non-constructive system of logic based on transfinite induction rather simpler than the system which we have described. In particular, it would seem that it should be possible to eliminate the necessity of stating explicitly the validity of definitions by primitive recursions, since this principle itself can be shown to be valid by transfinite induction. It is possible to make such modifications in the system, even in such a way that the resulting system is still complete, but no real advantage is gained by doing so. The effect is always, so far as I know, to restrict the class of formulae provable with a given set of axioms, so that we obtain no theorems but trivial restatements of the axioms. We have therefore to compromise between simplicity and comprehensiveness.

\section{Index of definitions.}

No attempt is being made to list heavy type formulae since their meanings are not always constant throughout the paper. Abbreviations

SER. 2. VOL. 45 . No. 2243. 
for definite well-formed formulae are listed alphabetically.

\begin{tabular}{|c|c|c|c|c|c|c|c|c|c|c|}
\hline & & & & Page & & & & & & Page \\
\hline$\ldots$ & $\ldots$ & $\ldots$ & $\ldots$ & 199 & Prod & & $\ldots$ & $\ldots$ & $\ldots$ & 207 \\
\hline $\mathrm{Al} \quad \ldots$ & $\ldots$ & $\ldots$ & $\ldots$ & 188 & $Q$ & & $\ldots$ & $\ldots$ & $\ldots$ & 176 \\
\hline $\mathrm{Bd} \ldots$ & $\ldots$ & $\ldots$ & $\ldots$ & 188 & Rec & & $\ldots$ & $\ldots$ & $\ldots$ & 222 \\
\hline Ck ... & $\ldots$ & $\ldots$ & $\ldots$ & 192 & Rt & $\ldots$ & $\ldots$ & $\ldots$ & $\ldots$ & 205 \\
\hline $\mathrm{Cm} \ldots$ & $\ldots$ & $\ldots$ & $\ldots$ & 222 & $\mathrm{~S}$ & $\ldots$ & $\ldots$ & $\ldots$ & $\ldots$ & 164 \\
\hline Comp & $\ldots$ & $\ldots$ & $\ldots$ & 201 & Sum & & $\ldots$ & $\ldots$ & $\ldots$ & 188 \\
\hline Dt $\ldots$ & $\ldots$ & $\ldots$ & $\ldots$ & 167 & $\mathrm{Sq}$ & $\ldots$ & $\ldots$ & $\ldots$ & $\ldots$ & 206 \\
\hline $\begin{array}{ll}E & \ldots\end{array}$ & $\ldots$ & $\ldots$ & $\ldots$ & 196 & $\mathrm{Tn}$ & $\ldots$ & $\ldots$ & $\ldots$ & $\ldots$ & 192 \\
\hline form & $\ldots$ & $\ldots$ & $\ldots$ & 166 & $\mathrm{Ug}$ & $\ldots$ & $\ldots$ & $\ldots$ & $\ldots$ & 188 \\
\hline $\begin{array}{lll}G & \ldots\end{array}$ & $\ldots$ & $\ldots$ & $\ldots$ & 1.96 & $V$ & $\ldots$ & $\ldots$ & $\ldots$ & $\ldots$ & 177 \\
\hline Gm ... & $\ldots$ & $\ldots$ & $\ldots$ & 214 & $\mathrm{Vi}$ & $\ldots$ & $\ldots$ & $\ldots$ & $\ldots$ & 210 \\
\hline $\operatorname{Gr} \quad \ldots$ & $\ldots$ & $\ldots$ & $\ldots$ & 166 & $W$ & $\ldots$ & $\ldots$ & $\ldots$ & $\ldots$ & 175 \\
\hline 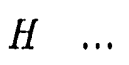 & $\ldots$ & $\ldots$ & 183 & 186 & $W^{\prime}$ & $\ldots$ & $\ldots$ & $\ldots$ & $\ldots$ & 176 \\
\hline$H_{1}$ & $\ldots$ & $\ldots$ & $\ldots$ & 186 & $X$ & $\ldots$ & $\ldots$ & $\ldots$ & $\ldots$ & 175 \\
\hline Hf $\ldots$ & $\ldots$ & $\ldots$ & $\ldots$ & 188 & $Z$ & $\ldots$ & $\ldots$ & $\ldots$ & $\ldots$ & 204 \\
\hline $\mathrm{Hg} \ldots$ & $\ldots$ & $\ldots$ & $\ldots$ & 206 & & & & & & \\
\hline$I \quad \ldots$ & $\ldots$ & $\ldots$ & $\ldots$ & 163 & $\Gamma$ & $\ldots$ & $\ldots$ & $\ldots$ & $\ldots$ & 177 \\
\hline $\operatorname{Inf} \ldots$ & $\ldots$ & $\ldots$ & $\ldots$ & 188 & $\delta$ & $\ldots$ & $\ldots$ & $\ldots$ & $\ldots$ & 162 \\
\hline $\mathrm{Jh} \quad \ldots$ & $\ldots$ & $\ldots$ & $\ldots$ & 204 & $\Theta$ & $\ldots$ & $\ldots$ & $\ldots$ & $\ldots$ & 210 \\
\hline$K \quad \ldots$ & $\ldots$ & $\ldots$ & $\ldots$ & 196 & $\Lambda_{G}{ }^{1}$ & $\ldots$ & $\ldots$ & $\ldots$ & $\ldots$ & 217 \\
\hline $\operatorname{Lim} \ldots$ & $\ldots$ & $\ldots$ & $\ldots$ & 188 & $\Lambda_{G}^{2}$ & $\ldots$ & $\ldots$ & $\ldots$ & $\ldots$ & 222 \\
\hline Ls $\ldots$ & $\ldots$ & $\ldots$ & $\ldots$ & 187 & $\Lambda_{G}{ }^{3}$ & $\ldots$ & $\ldots$ & $\ldots$ & $\ldots$ & 222 \\
\hline$M \quad \ldots$ & $\ldots$ & $\ldots$ & $\ldots$ & 222 & $\Lambda_{H}$ & $\ldots$ & $\ldots$ & $\ldots$ & $\ldots$ & 198 \\
\hline$M_{P} \ldots$ & $\ldots$ & $\ldots$ & $\ldots$ & 196 & $\Lambda_{P}$ & $\ldots$ & $\ldots$ & $\ldots$ & $\ldots$ & 196 \\
\hline $\mathrm{Mg} \ldots$ & $\ldots$ & $\ldots$ & $\ldots$ & 205 & $\varpi$ & $\ldots$ & $\ldots$ & $\ldots$ & $\ldots$ & 167 \\
\hline $\mathrm{Nm} \ldots$ & $\ldots$ & $\ldots$ & $\ldots$ & 177 & $\Sigma$ & $\ldots$ & $\ldots$ & $\ldots$ & $\ldots$ & 221 \\
\hline Od ... & $\ldots$ & $\ldots$ & $\ldots$ & 201 & 1,2 , & & $\ldots$ & $\ldots$ & $\ldots$ & 164 \\
\hline$P \quad \ldots$ & $\ldots$ & $\ldots$ & $\ldots$ & 188 & $\mathscr{P}$ & $\ldots$ & $\ldots$ & $\ldots$ & $\ldots$ & 170 \\
\hline
\end{tabular}

(The following refer to §§1-10 only.)

All-inclusive (logic formula) ... Axiomatic (class or property)... Circle-free

Computable function ... ...

Completeness, of class of logics of logic ... of ordinal logic
(Turing [1], 233)

Convertible 
Description number (D.N.) $\quad \ldots \quad \ldots \quad \ldots \quad \ldots \quad \ldots \quad$ (Turing [1], 240)

$\begin{array}{llllllllll}\text { Dual (W.F.F.) } & \ldots & \ldots & \ldots & \ldots & \ldots & \ldots & \ldots & \ldots & 170\end{array}$

$\begin{array}{lllllll}\text { Effectively calculable function } & \ldots & \ldots & \ldots & \ldots & \ldots & 166\end{array}$

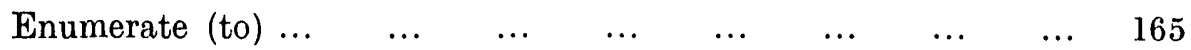

$\begin{array}{llllllll}\text { Formally definable function } & \ldots & \ldots & \ldots & \ldots & \ldots & \ldots & 165\end{array}$

$\begin{array}{llllllll}\text { General recursive function } & \ldots & \ldots & \ldots & \ldots & \ldots & \ldots & 166\end{array}$

$\begin{array}{llllllll}\text { Gödel representation (G.R.) } & \ldots & \ldots & \ldots & \ldots & \ldots & 165,166\end{array}$

$\begin{array}{llllllll}\text { Immediately convertible } & \ldots & \ldots & \ldots & \ldots & \ldots & \ldots & 1.62\end{array}$

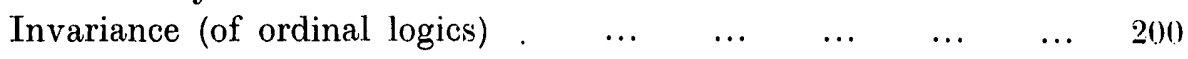

$\begin{array}{llllllllll}\text { Limit system } & \ldots & \ldots & \ldots & \ldots & \ldots & \ldots & \ldots & \ldots & 190\end{array}$

$\begin{array}{lllllllll}\text { Logic formula, Logic } & \ldots & \ldots & \ldots & \ldots & \ldots & \ldots & \ldots & 174\end{array}$

$\begin{array}{llllllllll}\text { Normal form } & \ldots & \ldots & \ldots & \ldots & \ldots & \ldots & \ldots & 162, & 165\end{array}$

$\begin{array}{llllll}\text { Number-theoretic (theorem or problem) } & \ldots & \ldots & \ldots & \ldots & 168\end{array}$

$\begin{array}{lllllllllll}\text { Oracle } & \ldots & \ldots & \ldots & \ldots & \ldots & \ldots & \ldots & \ldots & \ldots & 172\end{array}$

$\begin{array}{lllllllllll}\text { Ordinal } & \ldots & \ldots & \ldots & \ldots & \ldots & \ldots & \ldots & \ldots & \ldots & 178\end{array}$

$\begin{array}{llllllll}\text { Ordinal formula } & \ldots & \ldots & \ldots & \ldots & \ldots & \ldots & 179,180\end{array}$

$\begin{array}{lllllllll}\mathrm{C}-\mathrm{K} \text { ordinal formula } & \ldots & \ldots & \ldots & \ldots & \ldots & \ldots & \ldots & 181\end{array}$

$\begin{array}{llllllllll}\text { Ordinal logic } & \ldots & \ldots & \ldots & \ldots & \ldots & \ldots & \ldots & \ldots & 189\end{array}$

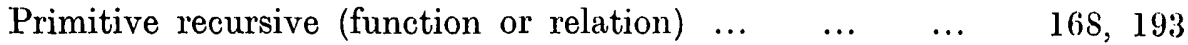

$\begin{array}{lllllllll}\text { Recursion formula } & \ldots & \ldots & \ldots & \ldots & \ldots & \ldots & \ldots & 193\end{array}$

Representation of ordinals, by ordinal formulae $\ldots$.. $\ldots$.

$\begin{array}{lllllllllll}\text { Type } & \ldots & \ldots & \ldots & \ldots & \ldots & \ldots & \ldots & \ldots & \ldots & 175 \\ & & \ldots & \ldots & \ldots & \ldots & \ldots & \ldots & 197\end{array}$

$\begin{array}{lllllllll}\text { Validity of system } & \ldots & \ldots & \ldots & \ldots & \ldots & \ldots & \ldots & 189\end{array}$

$\begin{array}{lllllll}\text { Well-formed formula (W.F.F.) } & \ldots & \ldots & \ldots & \ldots & \ldots & 162\end{array}$

$\begin{array}{lllllllll}\text { Well ordered series } & \ldots & \ldots & \ldots & \ldots & \ldots & \ldots & \ldots & 178\end{array}$

Miscellaneous (in order of appearance).

\begin{tabular}{|c|c|c|c|c|c|c|c|c|c|}
\hline & $\cdots$ & $\cdots$ & $\cdots$ & $\cdots$ & $\cdots$ & $\cdots$ & $\cdots$ & $\cdots$ & 163 \\
\hline$\lambda$-definable func & stion & $\ldots$ & $\ldots$ & $\ldots$ & $\ldots$ & $\ldots$ & $\ldots$ & $\ldots$ & 165 \\
\hline$G(x, y), D(x)$ & $\ldots$ & $\ldots$ & $\ldots$ & $\ldots$ & ... & $\ldots$ & $\ldots$ & $\ldots$ & 173 \\
\hline "<" between & W.F.F & & $\ldots$ & $\ldots$ & $\ldots$ & $\ldots$ & $\ldots$ & $\ldots$ & 181 \\
\hline$\Xi_{\mathrm{A}} \quad \ldots$ & $\ldots$ & ... & $\ldots$ & $\ldots$ & $\ldots$ & $\ldots$ & $\ldots$ & $\ldots$ & 182 \\
\hline Class $C$, system & ns $W$ & $\ldots$ & $\ldots$ & $\cdots$ & $\cdots$ & $\cdots$ & $\cdots$ & $\ldots$ & 189 \\
\hline$C[\mathbf{A}](\mathbf{A}$ a $\quad \mathbf{C}-\mathrm{K}$ & ordin: & & aula) & $\cdots$ & $\cdots$ & $\cdots$ & $\ldots$ & $\ldots$ & 190 \\
\hline System $P$ & $\cdots$ & $\cdots$ & $\ldots$ & $\cdots$ & $\ldots$ & $\ldots$ & (foot-note & $e \dagger)$ & 193 \\
\hline $\operatorname{Proof}_{C}\left[x_{6}, y_{0}\right]$ & $\ldots$ & $\ldots$ & ... & $\cdots$ & ... & $\cdots$ & $\ldots$ & $\ldots$ & 195 \\
\hline Systems $P_{\Omega}$ & $\ldots$ & $\cdots$ & $\ldots$ & $\cdots$ & $\ldots$ & $\ldots$ & $\ldots$ & $\ldots$ & 197 \\
\hline Systems $P^{\mathbf{A}}$ & $\cdots$ & $\ldots$ & $\ldots$ & $\ldots$ & ... & $\ldots$ & $\cdots$ & $\begin{array}{l}\ldots \\
\mathrm{Q} 2\end{array}$ & 210 \\
\hline
\end{tabular}




\section{Bibliography.}

Alonzo Church, [1]. "A proof of freedom from contradiction", Proc. Nat. Acad. Sci., 21 (1935), 275-281.

, [2]. Mathematical logic, Lectures at Princeton. University (1935-6), mimeo. graphed, $113 \mathrm{pp}$.

- [3]. "An unsolvable problem of elementary number theory", American $J$. of Math., 58 (1936), 345-363.

- [4]. "The constructive second number class", Bull. American Math. Soc., 44 (1938), 224-238.

G. Gentzen, [1]. "Die Widerspruchsfreiheit der reinen Zahlentheorie", Math. Annalen, 112 (1936), 493-565.

K. Gödel, [1]. "Über formal unentscheidbare Sätze der Principia Mathematica und verwandter Systeme, I", Monatshefte fïr Math. und Phys., 38 (1931), 173-189.

- [2]. On undecidable propositions of formal mathematical systems, Lectures at the Institute for Advanced Study, Princeton, N.J., 1934, mimeographed, $30 \mathrm{pp}$.

D. Hilbert, [1]. " Über das Unendliche ", Math. Annalen, 95 (1926), 161-190.

S. C. Kleene, [1]. "A theory of positive integers in formal logic ", American J. of Math., 57 (1935), 153-173 and 219-244.

- [2]. "General recursive functions of natural numbers", Math. Annalen, $112(1935-6), 727-742$.

- [3]. " $\lambda$-definability and recursiveness", Duke Math. Jour., 2 (1936), 340-353.

E. L. Post, [1]. " Finite combinatory processes-formulation 1 ", Journal Symbolic Logic, 1 (1936), 103-105.

J. B. Rosser, [1]. "Gödel theorems for non-constructive logics ", Journal Symbolic Logic, 2 (1937), 129-137.

A. Tarski, [1]. "Der Wahrheitsbegriff in den formalisierten Sprachen", Studia Philoso. phica, 1 (1936), 261-405 (translation from the original paper in Polish dated 1933).

A. M. Turing, [1]. "On computable numbers, with an application to the Entscheidungsproblem ", Proc. London Math. Soc. (2), 42 (1937), 230-265. A correction to this paper has appeared in the same periodical, 43 (1937), 544-546.

- [2]. "Computability and $\lambda$-definability", Journal Symbolic Logic, 2 (1937), $153-163$.

E. Zermelo, [1]. "Grundlagen einer allgemeiner Theorie der mathematischen Satzsysteme, I", Fund. Math., 25 (1935), 136-146.

Alonzo Church and S. C. Kleene, [1]. "Formal definitions in the theory of ordinal numbers", Fund. Math., 28 (1936), 11-21.

Alonzo Church and J. B. Rosser, [1]. "Some properties of conversion", Trans. American Math. Soc., 39 (1936), 472-482.

D. Hilbert and W. Ackermann, [1]. Grundzüge der theoretischen Logik (2nd edition revised, Berlin, 1938), $130 \mathrm{pp}$.

A. N. Whitehead and Bertrand Russell, [1]. Principia Mathematica (2nd edition, Cam. bridge, 1925-1927), 3 vols.

King's College,

Cambridge. 